\title{
Cyclin-Dependent Kinase (CDK) Inhibitors: Structure-Activity Relationships and Insights into the CDK-2 Selectivity of 6-Substituted 2-Arylaminopurines
}

\author{
Christopher R. Coxon, ${ }^{\dagger, \perp}$ Elizabeth Anscombe, ${ }^{\ddagger}$ Suzannah J. Harnor, ${ }^{\dagger}$ Mathew P. Martin, ${ }^{\S}$
}

Benoit Carbain, ${ }^{\dagger}$ Bernard T. Golding, ${ }^{\dagger}$ Ian R. Hardcastle, ${ }^{\dagger}$ Lisa K. Harlow, ${ }^{\dagger}$ Svitlana Korolchuk, ${ }^{\S}$ Christopher J. Matheson, ${ }^{\dagger}$ David R. Newell, ${ }^{\S}$ Martin E. M. Noble, ${ }^{\ddagger}, \|$ Mangaleswaran Sivaprakasam, ${ }^{\dagger}$ Susan J. Tudhope, ${ }^{\S}$ David M. Turner, ${ }^{\dagger}$ Lan Z. Wang, ${ }^{\S}$ Stephen R. Wedge, ${ }^{\S}$ Christopher Wong, Roger J. Griffin, ${ }^{\dagger, \#}$ Jane A. Endicott, ${ }^{*,+, \|}$ and Céline Cano ${ }^{*}, \dagger$

${ }^{\dagger}$ Newcastle Cancer Centre, Northern Institute for Cancer Research, School of Chemistry, Newcastle University, Bedson Building, Newcastle upon Tyne NE1 7RU, U.K.

${ }^{\ddagger}$ Department of Biochemistry, University of Oxford, South Parks Road, Oxford, OX1 3QU, U.K.

${ }^{\S}$ Newcastle Cancer Centre, Northern Institute for Cancer Research, Newcastle University Medical School, Paul O’Gorman Building, Framlington Place, Newcastle upon Tyne, NE2 4HH, U.K.
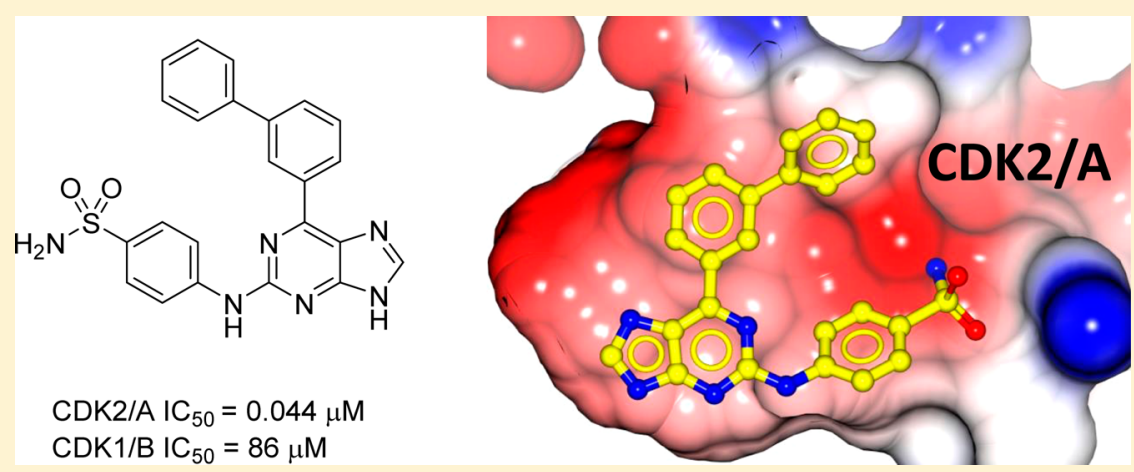

ABSTRACT: Purines and related heterocycles substituted at C-2 with 4 '-sulfamoylanilino and at C-6 with a variety of groups have been synthesized with the aim of achieving selectivity of binding to CDK2 over CDK1. 6-Substituents that favor competitive inhibition at the ATP binding site of CDK2 were identified and typically exhibited 10-80-fold greater inhibition of CDK2 compared to CDK1. Most impressive was 4-((6-([1,1'-biphenyl]-3-yl)-9H-purin-2-yl)amino) benzenesulfonamide (73) that exhibited high potency toward CDK2 $\left(\mathrm{IC}_{50} 0.044 \mu \mathrm{M}\right)$ but was $\sim 2000$-fold less active toward CDK1 (IC $56 \mu \mathrm{M}$ ). This compound is therefore a useful tool for studies of cell cycle regulation. Crystal structures of inhibitor-kinase complexes showed that the inhibitor stabilizes a glycine-rich loop conformation that shapes the ATP ribose binding pocket and that is preferred in CDK2 but has not been observed in CDK1. This aspect of the active site may be exploited for the design of inhibitors that distinguish between CDK1 and CDK2.

\section{INTRODUCTION}

The cyclin-dependent kinase (CDK) family of serine/threonine kinases plays an integral role in the regulation of the eukaryotic cell cycle as well as having key functions in apoptosis, transcription, differentiation, and neuronal function. ${ }^{1,2}$ Deregulation of the cell cycle, a hallmark of human cancer, is frequently associated with aberrant CDK activity through mechanisms that may include mutation/overexpression of CDKs or mutations to genes encoding proteins that directly or indirectly modulate $\mathrm{CDK}$ activity. ${ }^{3-5} \mathrm{CDK} 2$ activation entails association with partner cyclins $\mathrm{A}$ and $\mathrm{E}$, whereas the endogenous proteins $\mathrm{p} 21^{\mathrm{Cip} 1}$ and $\mathrm{p} 27^{\mathrm{Kip} 1}$ are inhibitory. Cyclin
E overexpression and/or $\mathrm{p} 27^{\mathrm{Kip} 1}$ suppression are a common feature of many human tumors. ${ }^{6,7}$

Pharmacological inhibition of CDK family members is a potential therapeutic approach for the treatment of cancer. In this context, a large number of ATP-competitive inhibitors have been evaluated. $^{5-9}$ The clinical development of CDK inhibitors as antitumor agents was originally hampered by poor kinase selectivity, particularly within the CDK family, and uncertainty as to which $\mathrm{CDK}$ constitutes the most appropriate therapeutic target. ${ }^{3}$ However, an appreciation of the roles played by specific

Received: August 23, 2016

Published: December 22, 2016 
CDKs in sustaining signaling through dysregulated pathways that drive particular cancers is leading to more stringent patient selection and correspondingly improved efficacy. For example, the highly selective CDK4/6 inhibitor palbociclib has been approved for the treatment of ER-positive, HER2-negative breast cancer, while abemaciclib, palbociclib, and ribociclib are in phase III clinical trials to treat advanced breast cancer in which signaling through the CDK4/6-retinoblastoma axis is critical. ${ }^{10}$ Similarly, agents that target a subset of CDKs are being evaluated in a range of other clinically defined segments.

The validity of CDK2 as a cancer therapeutic target was originally called into question both by CDK2 knockdown experiments in which loss of CDK2 failed to induce cell cycle arrest in a number of tumor cell lines ${ }^{12}$ and by mouse knockout experiments where the animals were viable. ${ }^{13,14}$ However, applying the rationale outlined above, CDK2 inhibitors are also expected to have utility in settings in which the cancer is addicted to enhanced CDK2 activity or where synthetic lethalities can be identified. Recent studies employing a chemical genetic approach in which $\mathrm{CDK} 2$ expression was maintained, but kinase activity was inhibited, provides compelling evidence that $\mathrm{CDK} 2$ is a valid cancer target. In a panel of human cancer cells transformed with various oncogenes, highly selective small-molecule CDK2 inhibition resulted in marked growth inhibition. ${ }^{15}$ CDK2-selective inhibitors may also have applications in combination therapies in appropriate clinical settings. For example, a recent study demonstrated that a combination of phosphatidylinositol-3kinase and CDK2 inhibitors induced apoptosis in malignant glioma xenografts via a synthetic-lethal interaction. ${ }^{16}$ Accumulating evidence also implicates $\mathrm{CDK} 2$ as a prospective therapeutic target in BRCA-deficient cancers, ${ }^{17}$ neuroblastoma, ${ }^{18}$ and ovarian cancer. ${ }^{19}$ These observations, taken together with supportive clinical data, ${ }^{20}$ have led to a resurgence of interest in CDK2 inhibitors as cancer therapeutic agents. ${ }^{21,22}$ Ideally, such an inhibitor would discriminate between CDK2 and CDK1 to avoid cell toxicity as a result of inhibiting CDK1, which is an essential member of the CDK family.

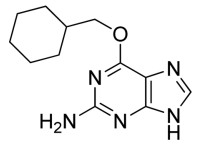

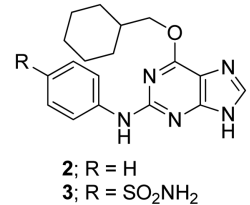

3; $\mathrm{R}=\mathrm{SO}_{2} \mathrm{NH}_{2}$

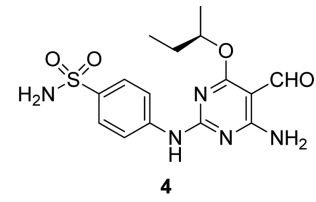

Our previous studies employing a structure-lead approach resulted in the development of selective ATP-competitive CDK2 inhibitors based on 2-amino-6-alkoxy- (1; NU2058) and 2-arylamino-6-alkoxy- (2) purine derivatives. ${ }^{23,24}$ These efforts were rewarded by the identification of potent purine-based inhibitors, exemplified by 6-cyclohexylmethoxy-2-(4'sulfamoylanilino) purine ( 3 ; NU6102, $\mathrm{CDK} 2 \quad \mathrm{IC}_{50}=5.0$ $\mathrm{nM})^{25}$ as well as alkoxypyrimidine inhibitors (e.g., 4, CDK2 $\left.\mathrm{IC}_{50}=0.8 \mathrm{nM}\right) .^{26}$ Importantly, 3 exhibits selectivity for CDK2 over other CDK-family members, proving some 50-fold selective for CDK2 over CDK1 $\left(\mathrm{IC}_{50}=250 \mathrm{nM}\right)$, a kinase with close structural homology to CDK2. In addition, 3 is only weakly active against $\mathrm{CDK} 7$ and $\mathrm{CDK} 9\left(\mathrm{IC}_{50}=4.4\right.$ and $1.1 \mu \mathrm{M}$, respectively). These CDKs play a key role in transcriptional regulation through phosphorylation of RNA polymerase II. ${ }^{2,27}$ Modulation of protein synthesis by off-target inhibition of
CDK7/CDK9 has obfuscated previous studies to elucidate the pharmacological effects of clinically evaluated compounds initially identified as selective CDK2 inhibitors such as seliciclib (5), dinaciclib (6), and SNS-032 (7). ${ }^{3,28,29}$

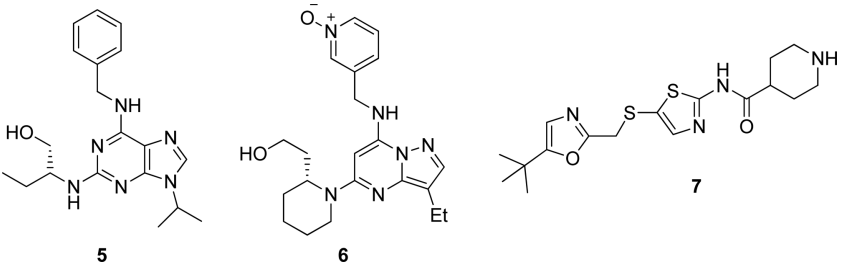

Crystal structures of purines $\mathbf{1 - 3}$ in complex with T160phosphorylated CDK2-cyclin A (CDK2-cyclin A) provided valuable details of inhibitor interactions within the ATP-binding site. $^{23,24}$ The purine heterocycle anchored the inhibitor through a triplet of hydrogen bonds between $N-9, N-3$, and the 2-amino group, and the backbone carbonyl moiety of Glu81 and amide and carbonyl moieties of Leu83, respectively, located in the hinge region of the kinase (Figure 2). The greater potency and selectivity of 3 compared with 2 and the parent 2-amino-6cyclohexylmethoxypurine 1 , has been attributed, at least in part, to additional interactions of the 2-sulfanilyl substituent of 3 with the specificity surface of CDK2. ${ }^{24,30}$ Notably, two hydrogen bond interactions between the sulfonamide group of 3 and Asp86 facilitate optimal hydrophobic packing of the arylamino ring.

Extensive structure-activity relationship studies (SARs) at the purine 2-position of 3 have further established the importance of these inhibitor-kinase interactions. ${ }^{25}$ By contrast, although the 6-cyclohexylmethyl group of 3 occupies the ribose-binding pocket, the precise nature of the interactions made by substituents at the purine 6-position with the CDK2 active site remains uncertain, notwithstanding that a large number of derivatives have previously been evaluated empirically. ${ }^{31}$

In this paper, we report the results of studies to characterize interactions between substituents at the purine 6-position and the CDK2 ATP-binding site. The effect upon biological activity of modifying the core purine heterocycle of 3 is also reported. These studies have resulted in the identification of $4-\left(\left(6-\left(\left[1,1^{\prime}-\right.\right.\right.\right.$ biphenyl]-3-yl)-9H-purin-2-yl)amino) benzenesulfonamide 73 as a potent and selective $\mathrm{CDK} 2$ inhibitor $\left(\mathrm{IC}_{50}=44 \mathrm{nM}\right)$, exhibiting some 2000-fold-selectivity over CDK1 $\left(\mathrm{IC}_{50}=86\right.$ $\mu \mathrm{M})$.

\section{CHEMISTRY}

With a view to establishing the overall contribution of the 6cyclohexylmethoxy substituent of $\mathbf{1}$ and $\mathbf{3}$ to inhibitor binding, the simple 6 -unsubstituted purines 8,12 , and 13 were required. 2-Aminopurine (8) was commercially available, while the 2phenylaminopurine (12) and 2-sulfanilylpurine (13) derivatives were synthesized as outlined in Scheme 1. Thus, selective reductive dehalogenation of 6-chloro-2-fluoropurine (10), prepared from 2-amino-6-chloropurine (9) following a literature procedure, ${ }^{32}$ afforded 2-fluoropurine (11). Treatment of 11 with aniline or 4-aminobenzenesulfonamide (sulfanilamide) in 2,2,2-trifluoroethanol (TFE) with catalysis by trifluoroacetic acid (TFA) as described previously ${ }^{33}$ gave the respective 2-arylaminopurines 12 and $\mathbf{1 3}$ in good yield. The required 2-arylaminoguanine derivatives 15 and 16 were also readily accessible from commercially available 2-bromohypox- 


\section{Scheme $1^{a}$}

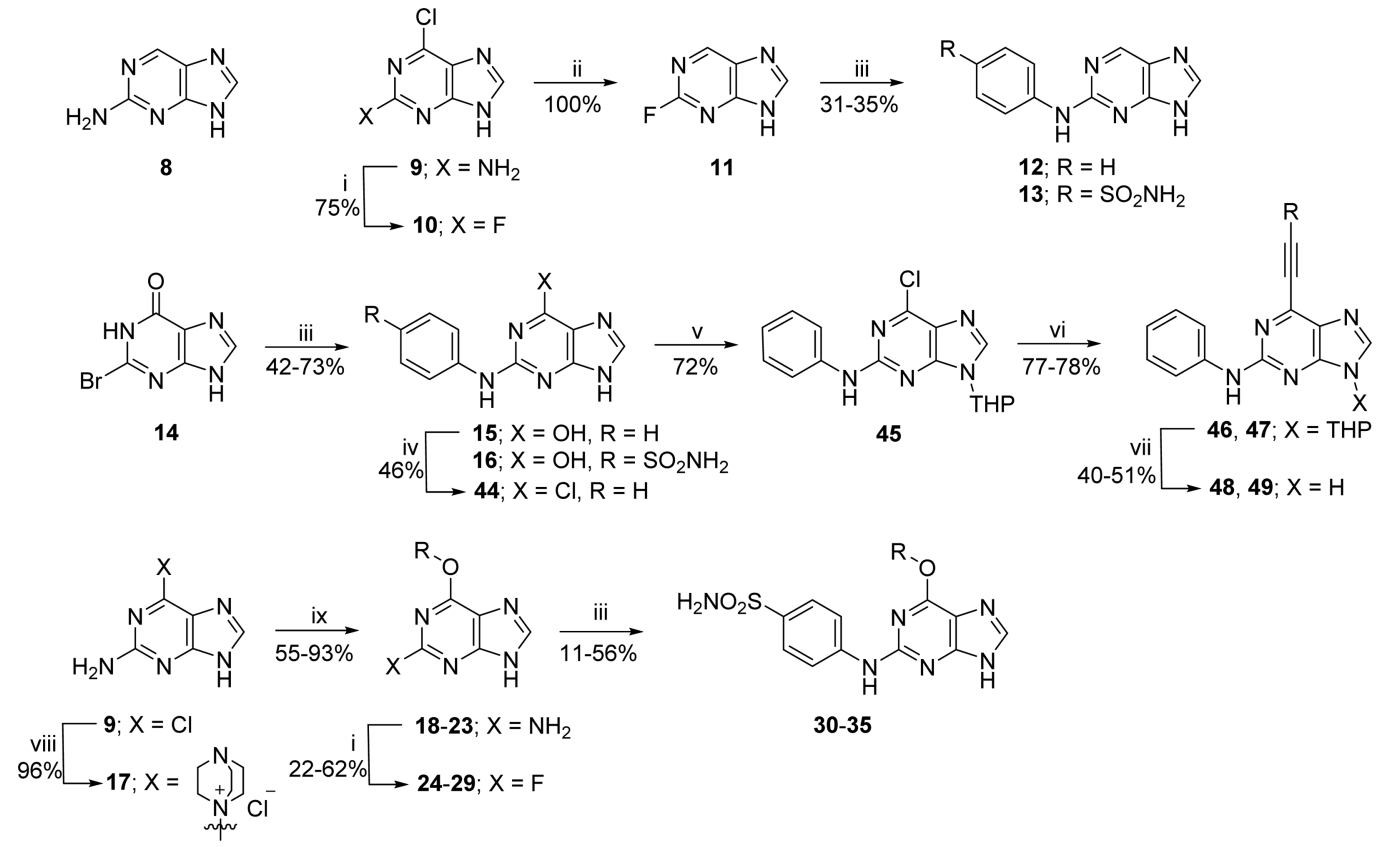

${ }^{a}$ Reagents and conditions: (i) aq $\mathrm{HBF}_{4}, \mathrm{NaNO}_{2},-15$ to $0{ }^{\circ} \mathrm{C}$; (ii) $\mathrm{Pd}(\mathrm{OH})_{2}, \mathrm{HCO}_{2} \mathrm{NH}_{4}, \mathrm{MeOH}$, reflux; (iii) $\mathrm{ArNH}_{2}, \mathrm{TFA}, \mathrm{TFE}, 90{ }^{\circ} \mathrm{C}$; (iv) POCl${ }_{3}$,

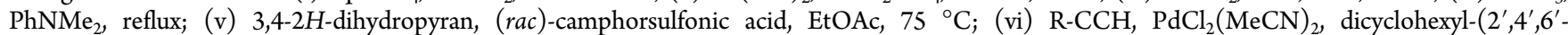
triisopropyl-[1,1'-biphenyl]-2-yl)phosphine, $\mathrm{Cs}_{2} \mathrm{CO}_{3}, \mathrm{MeCN}$; (vii) TFA, $i-\mathrm{PrOH}, \mathrm{H}_{2} \mathrm{O}$, reflux; (viii) DABCO, DMSO, $25^{\circ} \mathrm{C}$. (ix) For 18, 19, 22: $\mathrm{ROH}, \mathrm{Na}$, reflux. For 20, 21, 23: ROH, NaH, DMSO, $25{ }^{\circ} \mathrm{C}$. For definition of R groups, see Table 1.

anthine (14) under similar conditions. The 6-alkoxypurine derivatives (30-35) were synthesized by utilizing previously optimized methodology. ${ }^{23,25,31}$ Briefly, introduction of the 6alkoxy substituent was achieved either by direct reaction of the appropriate sodium alkoxide with 2-amino-6-chloropurine (9) by employing the corresponding alcohol as solvent or, where necessary, following conversion into the "DABCO-purine" intermediate (17). ${ }^{34}$ Elaboration of the 6-alkoxy-2-aminopurines (18-23) into the corresponding 2-fluoropurines (24-29) under Balz-Schiemann conditions enabled subsequent introduction of the 2-(4-aminobenzenesulfonamide) group using the TFA-TFE procedure to furnish the target purines (30-35) in good overall yields (Scheme 1).

The 6-ethynylpurine derivatives (41 and 42) were synthesized from the THP-protected 6-chloro-2-fluoropurine $(36)^{35}$ via a Sonogashira alkynylation approach (Scheme 2). Coupling of triisopropylacetylene with 36 , employing Pd$\left(\mathrm{PPh}_{3}\right)_{2} \mathrm{Cl}_{2} / \mathrm{CuI}$, afforded the protected 6-ethynylpurine (37) in excellent yield, and removal of the $N^{9}$-THP group to give 38 and introduction of the 2-arylamino substituent proceeded in the manner described above to give 39 and 40. Removal of the TIPS group to furnish the target 2-arylamino-6-ethynylpurines 41 and 42 was achieved under standard conditions. The 6propynyl- and 6-phenylethynyl-purine derivatives 48 and 49 were accessible from the guanine derivative 15 by sequential chlorination at the purine 6-position (44), THP protection (45), introduction of the appropriately substituted ethynyl group under Sonogashira conditions $(46,47)$, and final $N^{9}$ deprotection to furnish the target purines 48 and 49 (Scheme 1 ). The 6-ethylpurine derivative (52) was readily obtained by reduction of the 6-ethynyl group of the THP derivative 43 with Lindlar's catalyst-quinoline to afford 50, with $N^{9}$-deprotection (51) and final introduction of the 2-(4-aminobenzenesulfonamide) group, giving $\mathbf{5 2}$ in modest overall yield.

\section{Scheme $2^{a}$}

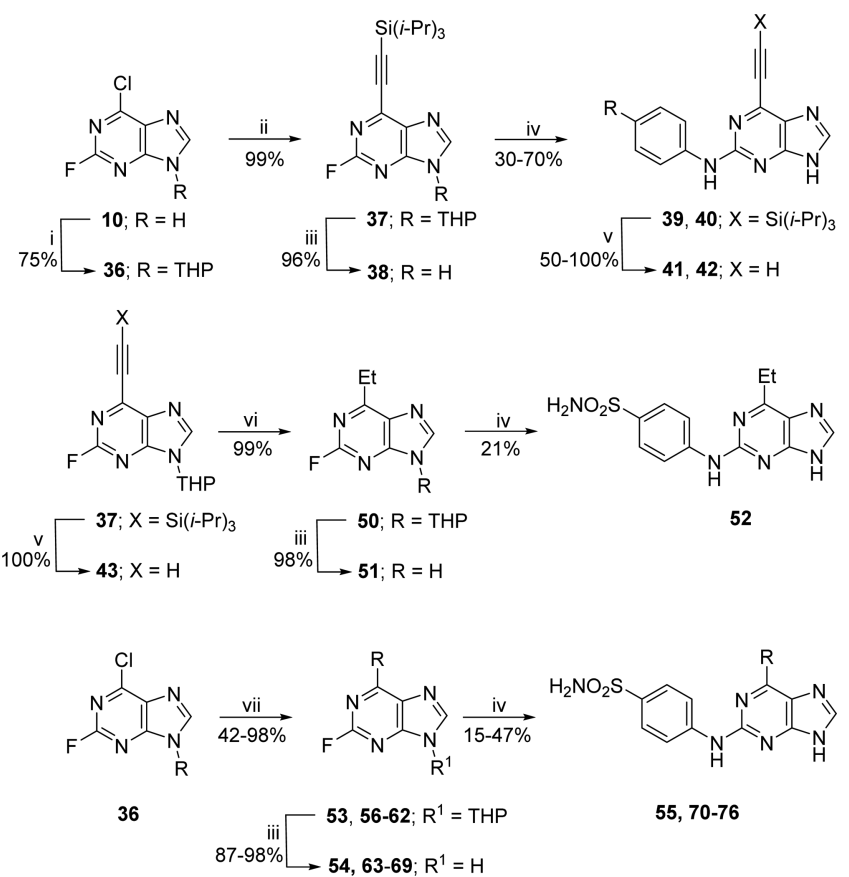

${ }^{a}$ Reagents and conditions: (i) 3,4-2H-dihydropyran, ( rac)-camphorsulfonic acid, EtOAc, $75{ }^{\circ} \mathrm{C}$; (ii) (i-Pr) $)_{3} \mathrm{SiCCH}, \mathrm{Pd}\left(\mathrm{PPh}_{3}\right)_{2} \mathrm{Cl}_{2}, \mathrm{CuI}$, $\mathrm{Et}_{3} \mathrm{~N}$, THF, $25^{\circ} \mathrm{C}$; (iii) TFA, $i$-PrOH, $\mathrm{H}_{2} \mathrm{O}$, reflux; (iv) $\mathrm{PhNH}_{2}$ or 4$\mathrm{NH}_{2} \mathrm{C}_{6} \mathrm{H}_{4} \mathrm{SO}_{2} \mathrm{NH}_{2}$, TFA, TFE, $90{ }^{\circ} \mathrm{C}$; (v) $(n-\mathrm{Bu})_{4} \mathrm{NF}$, THF, $25{ }^{\circ} \mathrm{C}$; (vi) Lindlar's catalyst, quinoline, $\mathrm{H}_{2}$, EtOAc, RT, $2 \mathrm{~h}$; (vii) $\mathrm{RB}(\mathrm{OH})_{2}$ or $\mathrm{RBF}_{3} \mathrm{~K}, \mathrm{Pd}(\mathrm{OAc})_{2}$, dicyclohexyl $\left(2^{\prime}, 6^{\prime}\right.$-dimethoxybiphenyl-2-yl)phosphine, $\mathrm{K}_{3} \mathrm{PO}_{4}, \mathrm{PhMe}, \mathrm{H}_{2} \mathrm{O}, 100{ }^{\circ} \mathrm{C}$. For definition of $\mathrm{R}$ groups, see Table 1. 
Scheme $3^{a}$

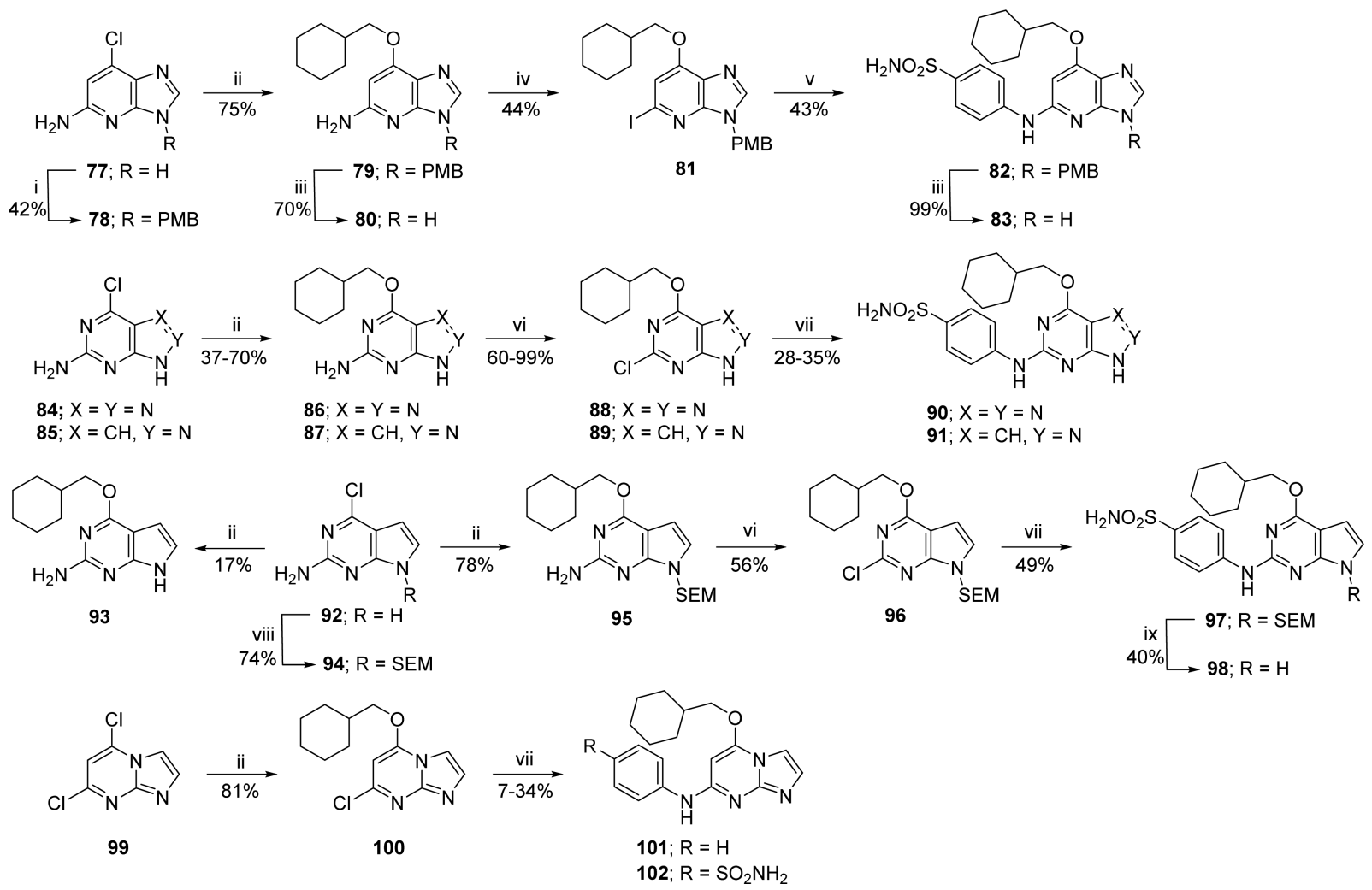

${ }^{a}$ Reagents and conditions: (i) PMB-Cl, $\mathrm{K}_{2} \mathrm{CO}_{3}$, DMF, $80{ }^{\circ} \mathrm{C}$; (ii) $\mathrm{NaH}$, cyclohexylmethanol, DMSO, $25{ }^{\circ} \mathrm{C}$; (iii) $\mathrm{TFA}, 80{ }^{\circ} \mathrm{C}$; (iv) $\mathrm{CH}_{2} \mathrm{I}_{2}$, iso-amyl nitrite, CuI, THF, $80{ }^{\circ} \mathrm{C}$; (v) sulfanilamide, $\mathrm{Pd}_{2}(\mathrm{dba})_{3}$, $\mathrm{XPhos}, \mathrm{K}_{2} \mathrm{CO}_{3}, \mathrm{MeCN}, 80{ }^{\circ} \mathrm{C}$; (vi) LiCl, iso-amyl nitrite, $\mathrm{SOCl}_{2}, \mathrm{DMA}^{\circ}$ or THF, $0-25{ }^{\circ} \mathrm{C}$; (vii) $\mathrm{ArNH}_{2}$, TFA, TFE, $90{ }^{\circ} \mathrm{C}$; (viii) SEM-Cl, NaH, MeCN, $0{ }^{\circ} \mathrm{C}$; (ix) TBAF, THF, $25{ }^{\circ} \mathrm{C}$.

Efforts to synthesize the 6-cyclopropylpurine (55) employing Suzuki-Miyaura conditions were unsuccessful, likely due to competing protodeboronation of the cyclopropylboronic acid under the reaction conditions employed. This problem was successfully addressed as reported in the literature by converting the cyclopropylboronic acid into the corresponding potassium trifluoroborate, ${ }^{36}$ whereby coupling to the THPprotected 6-chloro-2-fluoropurine (36) occurred to furnish 53 in acceptable yield. Subsequent transformation into the target 6-cyclopropylpurine (55) was achieved readily. The introduction of aryl substituents at the purine 6-position was achieved using Suzuki-Miyaura cross-coupling chemistry with 6-chloro2-fluoropurine derivative 36 (Scheme 2). Optimization of the reagents and reaction conditions employed for the crosscoupling step enabled the preparation of a defined series of 2fluoro-6-arylpurine derivatives (56-62) in excellent yields, and conversion into the requisite 6-aryl-2-(4-sulfonamidophenyl)aminopurines (70-76) proceeded smoothly under the previously described conditions. ${ }^{25,33}$

The synthesis of prospective CDK2 inhibitors encompassing alternative heterocyclic systems was undertaken as summarized in Scheme 3. In each case, the parent chloroheterocycles (77, 84, 85, 92, 99) were synthesized by adapting literature procedures $^{37-41}$ and the cyclohexylmethyl substituent introduced by treatment with the corresponding alkoxide, with $N^{9}$ protected where necessary, as described previously. Iodination $(81)^{42}$ or chlorination $(88,89,96)$ of the respective 2aminoheterocycles under Sandmeyer conditions facilitated introduction of the 2-arylamino group under the standard TFA-TFE conditions $(\mathbf{8 8}, \mathbf{8 9})$ or employing a Buchwald amination procedure $(\mathbf{8 1}, \mathbf{9 6})$, followed by deprotection as necessary to afford the target compounds 83, 90, 91, and 98 . The imidazo[1,2-a]pyrimidine derivatives $(101,102)$ were prepared from 5,7-dichloroimidazo[1,2-a]pyrimidine $(99)^{43,44}$ by regioselective 5 -alkoxylation to give 100, followed by final introduction of the arylamino group at the 7-position under TFA-TFE conditions. Given the possibility of alkoxide attack at the 7-position of 99 to furnish the alternative regioisomer, the structure of 102 was unambiguously confirmed by X-ray crystallography (Supporting Information).

\section{RESULTS AND DISCUSSION}

The chemical structures, CDK inhibitory activity, and in vitro antitumor activity for the purine derivatives and alternative heterocycles are summarized in Tables $1-3$, with the values for compounds 1-3 included for comparative purposes.

Structure-Activity Relationships. The importance of a substituent at the purine 6-position was reaffirmed by the simple 2-amino- and 2-phenylamino-purines ( 8 and 12), where a dramatic reduction in potency was observed compared with the parent 6-cyclohexylmethoxy derivatives 1 and 2, respectively. A similar effect was also evident for the sulfanilylpurine 13, which was some 300 -fold less active than 3 . These results are consistent with the putative interaction of this inhibitor class with the ATP-binding site of CDK2, which requires that the 6-substituent occupies a lipophilic pocket close to the ribose binding site, thereby orienting the purine to make the triplet of hydrogen bonds with the hinge region. ${ }^{24}$ The low-micromolar potency of the 2-sulfanilyl derivative 13 likely reflects the contribution to binding arising through additional interactions 
Table 1. Chemical Structures and CDK2-Inhibitory Activity of Purine Derivatives

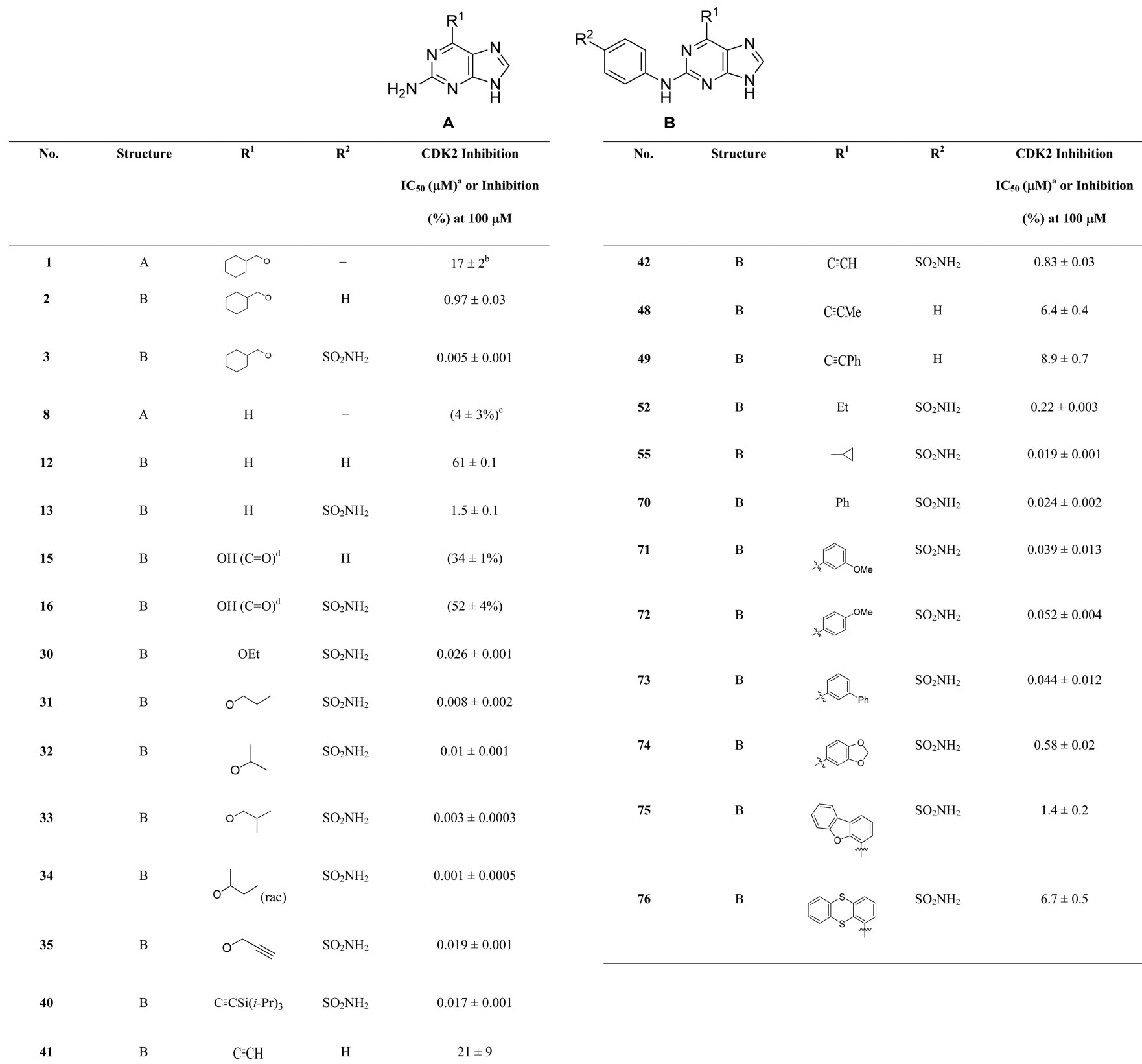

${ }^{a} \mathrm{IC}_{50}$ values were determined in accordance with previously described methods. ${ }^{20,23}{ }^{b}$ Data shown are the mean of at least two independent experiments \pm standard deviation. ${ }^{c}$ Percent inhibition values are in brackets. ${ }^{d}$ The oxo-tautomers predominate.

between the arylsulfonamide group of 13 with the CDK2 surface adjacent to the ATP binding site on the C-terminal lobe (termed the "specificity surface") despite the absence of a 6substituent. Perhaps not surprisingly, the guanine derivatives $\mathbf{1 5}$ and $\mathbf{1 6}$ were only weakly active, attributable to unfavorable interactions between the 6-oxo functionality and the ribosebinding pocket. These derivatives also exhibited the very poor aqueous solubility characteristic of many guanine derivatives.

Replacement of the 6-cyclohexylmethoxy group of 3 by smaller alkoxy substituents proved informative and was broadly consistent with our earlier investigations with derivatives of $\mathbf{1}^{31}$ In that study, 2-amino-6-methoxypurine ( $\mathrm{O}^{6}$-methylguanine) demonstrated negligible activity $\left(\mathrm{CDK} 2 ; \mathrm{IC}_{50}>100 \mu \mathrm{M}\right)$, and the relatively poor chemical stability of this purine militated against preparing the corresponding 2-sulfanilyl analogue. The 6-ethoxypurine 30 exhibited high CDK2 inhibitory activity $\left(\mathrm{IC}_{50}=26 \mathrm{nM}\right)$, with an approximately 3-fold increase in potency being observed for the $n$-propoxy- $\left(31 ; \mathrm{IC}_{50}=8 \mathrm{nM}\right)$ and iso-propoxy- $\left(32 ; \mathrm{IC}_{50}=10 \mathrm{nM}\right)$ derivatives. Potency was improved further by increasing the size of the 6-alkoxy group, as demonstrated by the activity of the isomeric butoxy analogues 33 and 34 with $\mathrm{IC}_{50}$ values of 3 and $1 \mathrm{nM}$, respectively. The relationship between the bulk of the 6-alkoxy group and the CDK2-inhibitory activity generally parallels that previously observed for the corresponding 2-amino-6-alkoxypurines, albeit that the sulfanilylpurines 30-34 are several orders of magnitude more potent. For example, $34\left(\mathrm{IC}_{50}=1\right.$ $\mathrm{nM})$ is approximately 50000 -fold more potent than the corresponding 2-amino-6-sec-butoxypurine (CDK2; $\mathrm{IC}_{50}=49$ $\pm 7 \mu \mathrm{M}){ }^{31}$ again corroborating the crucial binding 
contribution made by the arylsulfonamide function. A propargyloxy group at the purine $O^{6}$-position conferred activity comparable to the ethoxy derivative (compare 35 and 30), in keeping with the trend reported previously for the 2-amino-6alkoxypurine series. $^{31}$

The introduction of an ethyl substituent at the purine 6position (52) reduced CDK2-inhibitory activity some 10-fold compared with the 6-ethoxypurine (30), and very modest potency was also observed for the 6-ethynyl derivatives 41 and 42. Although 6-alkoxy and 6-alkyl groups differ with regard to both their electronic and steric character, as well as lipophilicity, the high potency of the 6-triisopropylsilyethynylpurine (40; $\mathrm{IC}_{50}=17 \mathrm{nM}$ ) shows that steric bulk, as well as electronic factors, is an important feature for binding in the ribose pocket of CDK2. The modest improvement in potency observed for the prop-1-ynyl (48) and phenylethynyl (49) derivatives compared with the parent 6-ethynylpurine 41 also suggests that the overall shape of the 6-substituent is an important factor for occupancy of the ribose-binding pocket, supported by the activity of the 6-cyclopropylpurine $\mathbf{5 5}\left(\mathrm{IC}_{50}=19 \mathrm{nM}\right)$. These data also suggest that the oxygen atom of the 6-alkoxypurines, which would act as a weak hydrogen bond acceptor, does not make a significant contribution to binding affinity.

The potency of the 6-phenylpurine (70; $\left.\mathrm{IC}_{50}=24 \mathrm{nM}\right)$ prompted further elaboration, with comparable activity residing in the 3-methoxyphenyl (71), 4-methoxyphenyl (72), and 6-(3phenylphenyl)purine (73) derivatives. By contrast, the piperonyl derivative (74) was some 20 -fold less potent than 70, and the introduction of still larger groups was detrimental, as evident from the very weak CDK2 inhibitory activity of the dibenzofuran-1-yl (75) and thianthren-1-yl (76) analogues. The bulkier bicyclic and tricyclic heteroaromatic rings of these purines are presumably poorly accommodated within the ribose-binding pocket. It is of interest to note that the CDK2 inhibitory activity of the 6-unsubstituted derivative $\mathbf{1 3}$ is equipotent with 75 and superior to that of 76 , implying that the 6-substituents of these purines make a negligible binding contribution.

With a view to assessing selectivity for CDK2, selected purine derivatives were evaluated against a panel of CDKs (Table 2). As expected, removal of the 6-cyclohexylmethyl substituent of 3 , which makes a number of interactions with the conserved ATP ribose binding site to furnish 13 compromised potency against all the CDKs evaluated, although the effect was less pronounced against CDK2.

The 6-alkoxypurines $\mathbf{3 4}$ and $\mathbf{3 5}$ exhibited good selectivity for CDK2 over the closely related CDK1 (80-fold and 50-fold, respectively), with 34 retaining selectivity over CDKs 4, 7, and 9 comparable with 3 . A similar profile was observed for the 6phenylpurine 70, which was some 30 -fold selective for CDK2 over CDK1. By contrast, the 6-([1,1'-biphenyl $]-3$-yl $)$ purine derivative 73 exhibited a very interesting CDK selectivity profile, proving some 2000 -fold selective for CDK2 over CDK1 while exhibiting only weak inhibitory activity against CDKs 4, 7, and 9. To the best of our knowledge, this level of selectivity for CDK2 over CDK1 is unprecedented.

Modification of the core purine heterocycle of $\mathbf{1}$ and 3 afforded further interesting results (Table 3). A comparison of 2-amino-6-cyclohexylmethylpurine $\mathbf{1}$ with the alternative heterocycles 86, 87, and 93 revealed that although these compounds exhibited comparable potency against CDK2, activity against other CDK family members was markedly attenuated. The very weak activity of the imidazopyrimidine
Table 2. CDK Selectivity and Cellular Activity for Selected Compounds

\begin{tabular}{|c|c|c|c|c|c|c|}
\hline \multirow[t]{2}{*}{ Compd. } & \multirow[t]{2}{*}{$\mathbf{R}$} & \multicolumn{5}{|c|}{ IC $_{50}(\mu \mathrm{M})$ or $\%$ inhibition at $100 \mu \mathrm{M}^{\mathrm{a}}$} \\
\hline & & CDK1/B & CDK2/A & CDK4/D & CDK7/H & CDK9/T \\
\hline 3 & & $\begin{array}{c}0.25 \pm \\
0.05^{\mathrm{b}}\end{array}$ & $\begin{array}{c}0.005 \pm \\
0.001\end{array}$ & $\begin{array}{c}1.45 \pm \\
0.70\end{array}$ & $\begin{array}{c}4.40 \pm \\
0.90\end{array}$ & $\begin{array}{c}1.07 \pm \\
0.02\end{array}$ \\
\hline 13 & $\mathrm{H}$ & $15.8 \pm 1.2$ & $1.5 \pm 0.09$ & $\begin{array}{l}(12 \pm \\
4 \%)^{\mathrm{c}}\end{array}$ & $75 \pm 3$ & $38 \pm 11$ \\
\hline 34 & & $\begin{array}{c}0.097 \pm \\
0.040\end{array}$ & $\begin{array}{c}0.0012 \pm \\
0.0005\end{array}$ & $\begin{array}{c}1.30 \pm \\
0.30\end{array}$ & $2.8 \pm 0.1$ & $\begin{array}{c}0.57 \pm \\
0.05\end{array}$ \\
\hline 35 & & $0.94 \pm 0.1$ & $\begin{array}{c}0.019 \pm \\
0.001\end{array}$ & $8.8 \pm 1.2$ & - & - \\
\hline 70 & $\mathrm{Ph}$ & $\begin{array}{l}0.73 \pm \\
0.003\end{array}$ & $\begin{array}{c}0.024 \pm \\
0.002\end{array}$ & $3.8 \pm 1.4$ & $6.8 \pm 0.4$ & $\begin{array}{c}0.57 \pm \\
0.04\end{array}$ \\
\hline 73 & & $86 \pm 4$ & $\begin{array}{c}0.044 \pm \\
0.012\end{array}$ & $(26 \pm 2 \%)$ & $(28 \pm 6 \%)$ & $25 \pm 8$ \\
\hline
\end{tabular}

${ }^{a} \mathrm{IC}_{50}$ values were determined in accordance with previously described methods. ${ }^{20,23}{ }^{b}$ Data shown are the mean of at least two independent experiments \pm standard deviation. ${ }^{c}$ Percent inhibition values are in brackets.

derivatives 101 and 102 is not surprising given the absence of the requisite donor-acceptor-donor motif and presumably reflects the combined influence arising from the absence of the $N^{1}$ and $N^{7}$ functions found in the corresponding purine derivatives 1 and 3 . The modest $\mathrm{CDK}$ inhibition profile of imidazopyridine 83 indicates that removal of the $N^{1}$ nitrogen is detrimental to CDK inhibitory activity. However, with the exception of the imidazopyridine (83) and imidazopyrimidine (102) derivatives, modification of the core purine heterocycle of 3 was generally tolerated without a marked loss of CDK2 inhibitory activity. Thus, triazolopyrimidine (90) analogue is 2.5-fold more potent than 3, while pyrazolopyrimidine (91) and pyrrolopyrimidine (98) analogues proved only some 4-5fold less potent than 3 against CDK2 and retained good selectivity over all other CDK family members examined. Notably, derivatives 91 and 98 were approximately 100-fold selective for CDK2 over CDK1.

Cellular Studies. The ability of compounds to inhibit cellular proliferation was examined in a 5-day growth assay using five histologically distinct retinoblastoma protein $(\mathrm{Rb})$ proficient human tumor lines (A375 melanoma, Calu-6 lung carcinoma, MDA-MB-231 breast adenocarcinoma, SJSA1 osteosarcoma, and SKUT-1B uterine corpus leiomyosarcoma). While compound 73 demonstrated appreciable nanomolar potency versus $\mathrm{CDK} 2$ in isolated kinase assays, the maximal concentration tested in cellular assays $(30 \mu \mathrm{M})$ had no or limited effects on the growth of cells, thereby preventing the concentration that induced a $50 \%$ growth inhibitory $\left(\mathrm{GI}_{50}\right)$ effect to be determined (data not shown). In contrast, compounds 91 and 98, which were only 2.4- and 1.7-fold more potent respectively in CDK2 kinase assays, dosedependently inhibited the growth of each tumor cell line with $\mathrm{GI}_{50}$ values ranging from $1-22 \mu \mathrm{M}$. The cellular activity of 
Table 3. Chemical Structures and CDK Inhibitory Activity of Other Heterocyclic Derivatives

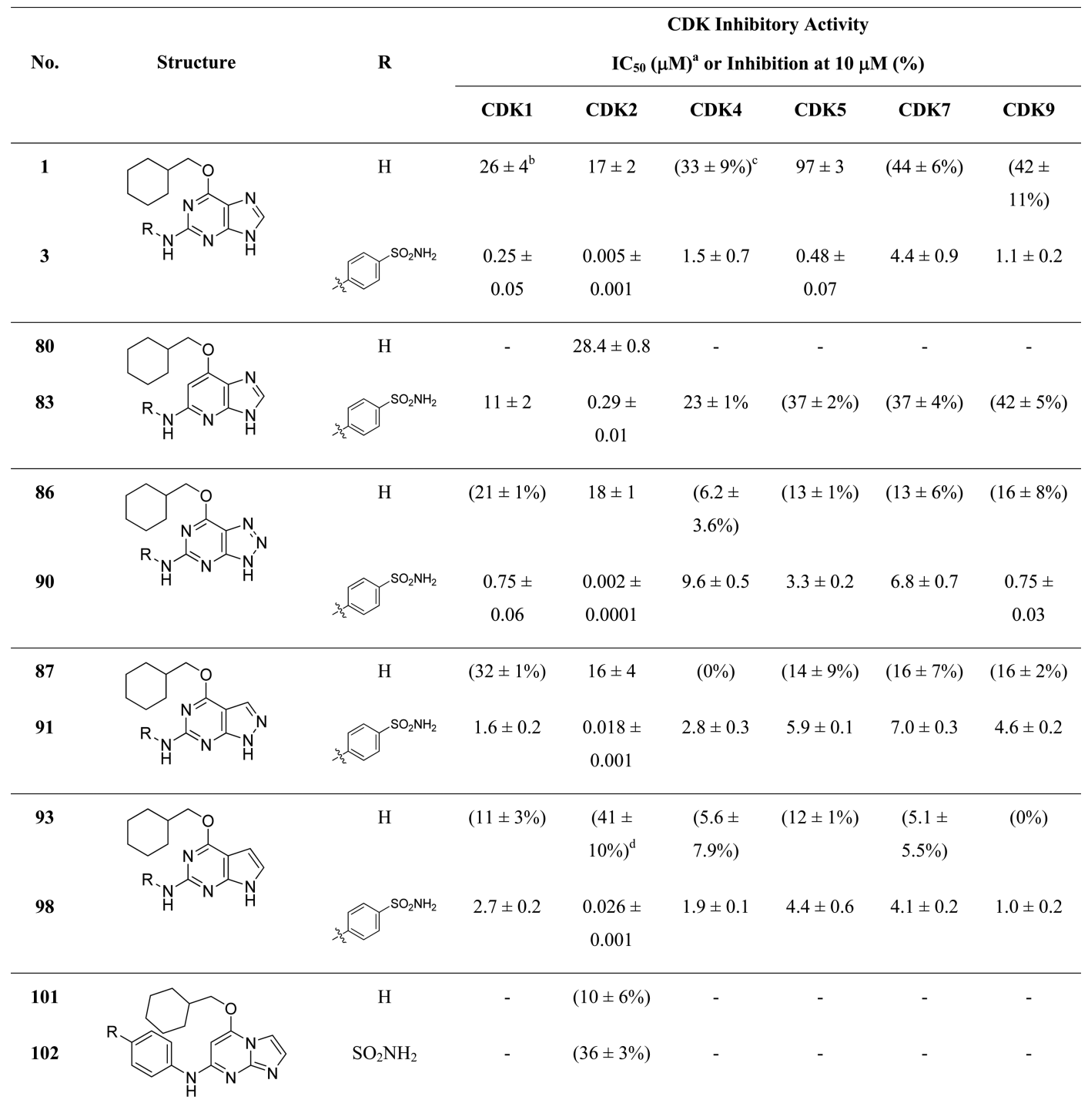

${ }^{a} \mathrm{IC}_{50}$ values were determined in accordance with previously described methods. ${ }^{20,23}{ }^{b}$ Data shown are the mean of at least two independent experiments \pm standard deviation. ${ }^{c}$ Percent inhibition values are in brackets. ${ }^{d}$ Value determined at $100 \mu \mathrm{M}$.

these compounds compared favorably to that of compound 3 (Figure 1).

Structure Determination. Compounds within this series show greater activity toward CDK2 than CDK1, generally varying between 10 - and 80-fold (Table 2). However, 73 exhibits exceptional discrimination being a ca. 2000-fold more potent inhibitor of CDK2. To confirm the binding mode and to identify potential interactions that might explain the selectivity of 73 for CDK2 over CDK1, 73 and 3 were cocrystallized with CDK2-cyclin A and CDK1-cyclin B-cyclin-dependent kinases regulatory subunit 2 (CKS2), respectively. The poor potency of 73 toward CDK1 appears to preclude determination of a CDK1-cyclin B-CKS2-73 cocomplex structure, as despite repeated attempts we were unable to visualize the compound in the active site. The statistics for the data sets and for the crystallographic refinements are presented in Table 4. Compound 73 emulates the interactions made by 3 within the CDK2 active site (Figure 2A). ${ }^{24}$ Indeed, the purine and aniline rings of each inhibitor superimpose very well. The purine rings occupy the CDK2 ATP binding site, and each makes a triplet of hydrogen bonds with the backbone carbonyl of Glu81 and the amide $\mathrm{NH}$ and carbonyl moieties of Leu83 within the hinge sequence (Figure 2A). The respective anilino rings project out of the $\mathrm{CDK} 2$ catalytic site so that the sulfonamide $\mathrm{NH}_{2}$ group and one of the oxygens in each make hydrogen bonds with the side chain and backbone nitrogen of Asp86, respectively.

The structures of 73 and 3 differ in the substitution present at the purine C-6 position. The $O^{6}$-cyclohexylmethyl substituent of 3 occupies the ATP ribose binding pocket and is complementary in shape and forms favorable hydrophobic interactions with an apolar pocket created by the conformation of the CDK2 glycine-rich loop (residues 9-19). ${ }^{24}$ This loop adopts an identical backbone structure when bound to 73 so that the purine-proximal phenyl ring emulates the position of the cyclohexylmethyl substitutent of 3 . The distal phenyl ring substituted at the meta position can then be comfortably accommodated as it twists toward the aniline moiety (Figure 2A). In contrast, para substitutions of the purine-proximal

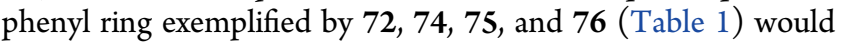



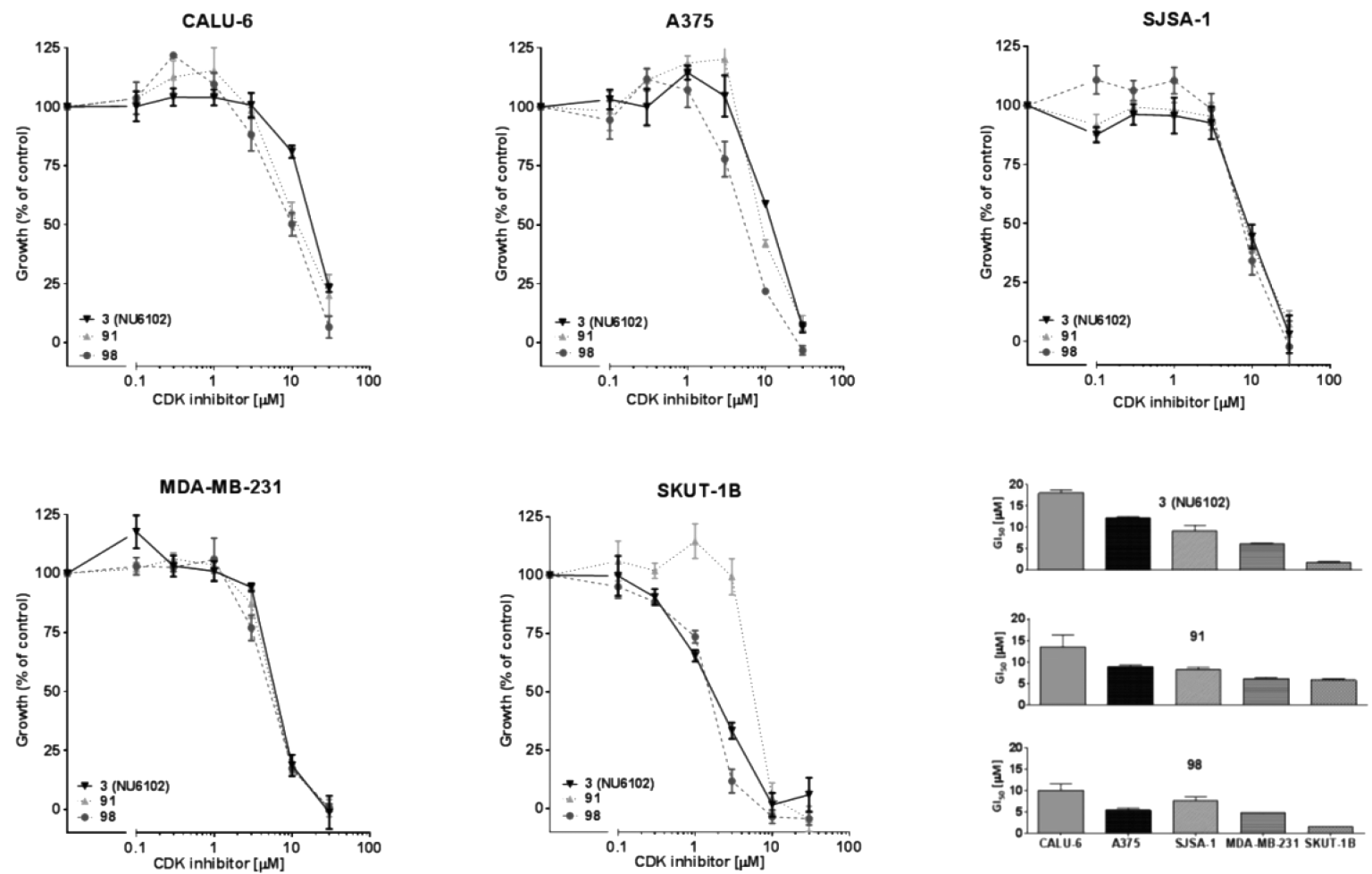

Figure 1. Activity of compounds 3, 91, and 98 against tumor cell growth. ${ }^{45} \mathrm{Rb}$-proficient human tumor cell lines were incubated for $120 \mathrm{~h}$ with compounds and cellular protein determined by SRB assay. Dose-response curves and $\mathrm{GI}_{50}$ bar charts represent the mean $\pm \mathrm{SEM}$ from three or four independent experiments.

Table 4. X-ray Data Collection and Refinement Statistics

\begin{tabular}{|c|c|c|}
\hline & $\begin{array}{c}\text { CDK2-cyclin A- } \\
\mathbf{7 3}^{a}\end{array}$ & CDK1-cyclin B-CKS2-3 ${ }^{a}$ \\
\hline \multicolumn{3}{|l|}{ Data Collection } \\
\hline space group & $P 2_{1} 2_{1} 2_{1}$ & $P 1$ \\
\hline unit cell $(\AA ̊)$ & $\begin{array}{l}a=73.5 \\
b=132.1 \\
c=149.2\end{array}$ & $\begin{array}{c}a=65.0, b=67.8, c=85.1, \\
\alpha=103.9, \beta=90.9, \gamma=90.4\end{array}$ \\
\hline resolution $(\AA)$ & $66.03-2.97$ & $65.76-2.06$ \\
\hline $\begin{array}{l}\text { (highest } \\
\text { resolution shell) }\end{array}$ & $(3.13-2.97)$ & $(2.10-2.06)$ \\
\hline total observations & $226370(7244)$ & 169709 (8389) \\
\hline unique & 30773 (1098) & $84841(4161)$ \\
\hline$R_{\text {merge }}{ }^{\mathrm{a}}$ & 0.086 & 0.092 \\
\hline mean $I / \sigma(I)$ & $6.7(1.6)$ & $6.2(1.2)$ \\
\hline completeness \% & $100(99.7)$ & $97.3(95.5)$ \\
\hline \multicolumn{3}{|l|}{ Refinement } \\
\hline \multicolumn{3}{|l|}{$\begin{array}{l}\text { total number of } \\
\text { atoms }\end{array}$} \\
\hline protein & 8927 & 20449 \\
\hline other & 64 & 100 \\
\hline waters & 18 & 291 \\
\hline $\begin{array}{l}R \text { (highest } \\
\quad \text { resolution shell) }\end{array}$ & 0.213 & 0.198 \\
\hline $\begin{array}{l}R_{\text {free }} \text { (highest } \\
\text { resolution shell) }\end{array}$ & 0.266 & 0.254 \\
\hline rmsd bonds $(\AA ̊)$ & 0.0122 & 0.0217 \\
\hline rmsd angles (deg) & 1.770 & 2.233 \\
\hline
\end{tabular}

${ }^{a}$ The structures have been deposited in the PDB with accession codes 5LQE (CDK2-cyclin A-73) and 5LQF (CDK1-cyclin B-CKS2-3).

be directed out of the CDK2 active site toward the tip of the glycine-rich loop. Smaller substitutions, for example a methoxy in 72 can be accommodated but larger ring systems as exemplified by 74 and 76 lead to considerable drops in potency, suggesting they may sterically clash with the CDK2 structure in this region (Table 1 ).

To probe further the binding mode of the series, CDK1cyclin B-CKS2 was cocrystallized with 3 (Figure 2B). This structure shows that the purine backbone emulates the interactions made by this inhibitor within the CDK2 binding site and that the $O^{6}$-cyclohexylmethyl substituent occupies the ribose binding pocket. As previously reported, ${ }^{46}$ inhibitor binding within the ATP binding site is accompanied by minor remodelling of the $\mathrm{CDK} 1$-cyclin $\mathrm{B}$ into a conformation compatible with catalysis.

All of the side chains contacted by 73 in the co-complex with CDK2-cyclin A are conserved in CDK1 so that selectivity must derive from indirect readout of remote sequence differences. One mechanism by which such differences can impact inhibitor binding is where they shape the conformational energy landscape, permitting conformations in one kinase that are precluded in another. As described above, the glycine-rich loop makes a significant contribution to the catalytic cleft, shaping the binding site that accommodates the purine C-6 substituents. We note that the glycine-rich loop in the CDK2-cyclin A-73 structure adopts a conformation in which Tyr15 is folded into the active site, contacting residues of the C-helix (Figure 2C). This position for the side chain of Tyr15 is seen in a number of other CDK2-cyclin A-inhibitor complexes, suggesting that it reflects a preferred conformation of CDK2. However, in available structures of apo CDK1-cyclin B, and CDK1-cyclin bound to compound $23^{46}$ or 3 (this paper), a comparable location for the side chain of Tyr 15 has not been observed (Figure 2C). We speculate, therefore, that 73 binds more tightly to CDK2 than to CDK1 because in doing so, it 


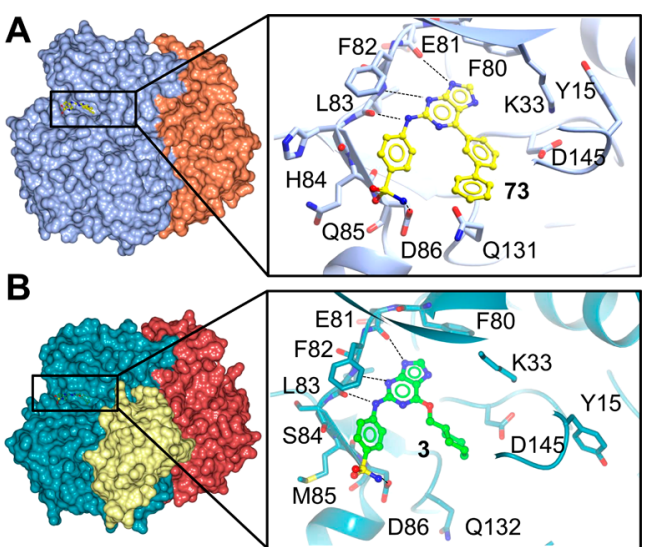

$\mathrm{c}$

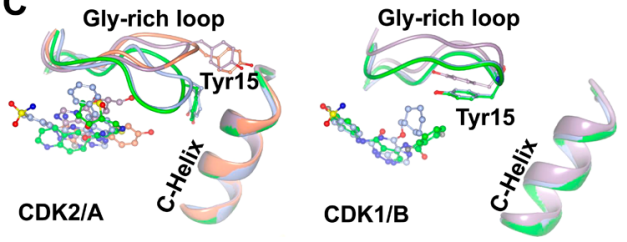

Figure 2. Structures of CDK2-cyclin A-73 and CDK1-cyclin BCKS2-3. (A) Surface representation of CDK2 (ice-blue) bound to cyclin A (coral) in complex with 73 (yellow). The inset shows the interactions of 73 within the ATP site of CDK2-cyclin A. (B) Surface representation of CDK1 (dark-cyan) bound to cyclin B (pale-crimson) and CKS2 (lemon) in complex with 3 (green). The inset shows the interactions of 3 within the ATP site of CDK1-cyclin B-CKS2. In (A) and (B), the protein backbone is rendered in ribbon representation and selected $\mathrm{CDK} 2$ and $\mathrm{CDK} 1$ residues are drawn respectively with carbon atoms colored ice-blue and dark-cyan. The hydrogen bonds are shown as black dotted lines. (C) The structural conformations of the CDK2-cyclin A and CDK1-cyclin B-CKS2 glycine-rich loops to illustrate the alternative poses of catalytic residue Tyr15. The left-hand side panel compares CDK2-cyclin A in complex with 73 (ice-blue) to other inhibitor bound CDK2-cyclin A complexes (PDB entries 4EOS (green), 3TNW (coral), and 3MY5 (lilac)). The right-hand panel overlays CDK1-cyclin B-CKS2 bound to 3 (ice-blue) with apo CDK1cyclin B-CKS2 (PDB entry 4YC3, lilac) and CDK1-cyclin B-CKS2 in complex with compound 23 (PDB entry 5HQ0, green). The conformations of the loop when CDK1 is bound to 3 or compound 23 cannot be distinguished. Gly-rich loop, glycine-rich loop.

stabilizes a glycine-rich loop conformation that is preferred in CDK2 but not in CDK1.

\section{CONCLUSIONS}

Novel 6-substituted 2-(4'-sulfamoylanilino)purines have been designed as competitive inhibitors acting at the ATP binding site of CDK2 with particular attention being given to abrogating activity against CDK1. A variety of substituents were explored, either attached directly to C- 6 or via an oxygen link, in the context of their possible interaction with a lipophilic pocket close to the ATP ribose binding site. The relationship between the size of a 6-alkoxy group and CDK2-inhibitory activity was found to parallel that previously observed for the corresponding 2-amino-6-alkoxypurines. In general, the new compounds were significantly less potent (typically $10-80 \times$ ) against CDK1 than CDK2. Most impressive was $4-\left(\left(6-\left(\left[1,1^{\prime}-\right.\right.\right.\right.$ biphenyl]-3-yl)-9H-purin-2-yl)amino) benzenesulfonamide that was $\sim 2000$-fold less active toward CDK1 $\left(\mathrm{IC}_{50} 86 \mu \mathrm{M}\right)$, while retaining high potency against CDK2 $(0.044 \mu \mathrm{M})$. Compounds substituted with relatively large conformationally constrained groups, e.g. bicyclic and tricyclic aromatic systems, showed greatly reduced inhibitory activity, indicating poorly accommodation of these substituents in the lipophilic binding site. Analogues of 6-cyclohexylmethoxy-2-(4'-sulfamoylanilino)purine, in which the purine ring was replaced by a triazolopyrimidine, pyrazolopyrimidine or pyrrolopyrimidine were only marginally less active against CDK2 than the parent purine. However, replacement with an imidazopyridine or imidazopyrimidine gave much less potent derivatives. Cocrystal structures of inhibitors bound to CDK2 and CDK1 revealed that the binding mode of the purine-based inhibitor series is conserved. We show that inhibitor binding to CDK2 stabilizes a glycine-rich loop conformation that shapes the ATP ribose binding pocket, resulting in effective inhibition of CDK2. We propose that this region of the active site might be the basis of the design of further inhibitors differentiating between CDK1 and CDK2.

\section{EXPERIMENTAL SECTION}

General Synthetic Procedures. Chemicals and solvents were obtained from standard suppliers. Solvents were either dried by standard techniques or purchased as anhydrous. Reactions needing microwave irradiation were carried out in an Initiator Sixty Biotage apparatus. Petrol refers to petroleum ether (bp $40-60{ }^{\circ} \mathrm{C}$, reagent grade, Fisher Scientific). All reactions that required inert or dry atmosphere were carried out under a blanket of nitrogen, which was dried by passage through a column of phosphorus pentoxide. Glassware was dried in an oven prior to use. Column chromatography was carried out using 40-60 $\mu \mathrm{m}$ mesh silica in glass columns under medium pressure or with a Biotage SP4 flash purification system using KP-Si. Thin layer chromatography (TLC) was performed on $20 \mathrm{~mm}$ precoated plates of silica gel (Merck, silica gel 60F254); visualization was achieved using ultraviolet light $(254 \mathrm{~nm})$. NMR spectra were recorded on a Bruker Spectrospin AC 300E (300 MHz) NMR Spectrometer or Bruker BioSpin UltraShield Plus $500 \mathrm{MHz}$ using deuterated solvent as a lock. IR spectra were recorded on a Bio-Rad FTS 3000MX diamond ATR, and UV analysis was performed using a Hitachi U-2000 spectrophotometer. LC-MS analysis was carried out on a Micromass Platform instrument operating in positive and negative ion electrospray mode, employing a $50 \mathrm{~mm} \times 4.6 \mathrm{~mm} \mathrm{C} 18$ column (Supelco Discovery or Waters Symmetry) and a $15 \mathrm{~min}$ gradient elution of $0.05 \%$ formic acid and methanol (10-90\%). HRMS were measured using a Finnigan MAT 95 XP or a Finnigan MAT 900 XLT by the EPSRC National Mass Spectrometry Service Centre (Swansea). The purity of final compounds was assessed by reversed-phase HPLC; all tested compounds were $>95 \%$ purity. HPLC instrument, Agilent 1200 equipped with a photodiode array detector $(190-400 \mathrm{~nm})$. Sample temperature, ambient; injection volume, $5 \mu \mathrm{L}$; flow rate, $1 \mathrm{~mL} / \mathrm{min}$. $5-100 \% \mathrm{MeCN}$ gradient over $9 \mathrm{~min}$ and an isocratic hold at $100 \% \mathrm{MeCN}$ for $2.5 \mathrm{~min}$, before returning to initial conditions. Mobile phase $\mathrm{A}=0.1 \%$ ammonia in water or $0.1 \%$ formic acid in water, mobile phase $\mathrm{B}=\mathrm{MeCN}$. Column: Waters XSELECT CSH C18, $3.5 \mu \mathrm{m}, 4.6 \mathrm{~mm} \times 150 \mathrm{~mm}$ or Waters XTerra RP18, $5 \mu \mathrm{m}$, $4.6 \mathrm{~mm} \times 150 \mathrm{~mm}$. Column maintained at ambient temperature.

General Procedure A. To a stirred suspension of the appropriate haloheterocycle ( $1.0 \mathrm{~mol}$ equiv) and 4-aminobenzenesulfonamide $(2.0$ mol equiv) in TFE $(25 \mathrm{~mL} / \mathrm{g}$ of haloheterocycle) was added TFA ( 2.5 $\mathrm{mL} / \mathrm{g}$ of haloheterocycle) dropwise. The resulting solution was heated under reflux for $12-48 \mathrm{~h}$ under a nitrogen atmosphere. The solvent was removed in vacuo, and the residue was redissolved in EtOAc (10$20 \mathrm{~mL}$ ). The solution was washed with saturated aqueous sodium bicarbonate solution $(3 \times 10 \mathrm{~mL})$, and the aqueous extracts were combined and washed with EtOAc $(2 \times 15 \mathrm{~mL})$. The combined organic layers were dried $\left(\mathrm{Na}_{2} \mathrm{SO}_{4}\right)$ and the solvent removed under reduced pressure to give a residue that was purified in the manner indicated.

6-Chloro-2-fluoro-9H-purine (10). ${ }^{47}$ To a stirred solution of $\mathrm{HBF}_{4}$ (48\% aqueous, $120 \mathrm{~mL}$ ) at $0{ }^{\circ} \mathrm{C}$ was added 2-amino-6-chloropurine (9) $(6.0 \mathrm{~g}, 35.0 \mathrm{mmol})$. Over $20 \mathrm{~min}$, a solution of $\mathrm{NaNO}_{2}(4.9 \mathrm{~g}, 70.0$ 
$\mathrm{mmol})$ in water $(200 \mathrm{~mL})$ was added dropwise, ensuring the temperature remained close to $0{ }^{\circ} \mathrm{C}$. The pale-yellow solution was raised to room temperature and stirred for $18 \mathrm{~h}$. The resulting solution was neutralized to $\mathrm{pH} 7$ in an ice bath at $0{ }^{\circ} \mathrm{C}$ by addition of $\mathrm{Na}_{2} \mathrm{CO}_{3}$ $(6.00 \mathrm{~g})$ in water $(200 \mathrm{~mL})$. Solvents were removed in vacuo, and the residual solid was redissolved in $\mathrm{MeOH}(100 \mathrm{~mL})$ and adsorbed onto silica $(250 \mathrm{~mL})$. The crude material was purified by chromatography (silica; $10 \% \mathrm{MeOH}: \mathrm{DCM}$ ) to afford $\mathbf{1 0}$ as a white crystalline solid (4.52 g, 75\%); mp 171-173 ${ }^{\circ} \mathrm{C}$ (lit., $\left.{ }^{47} \mathrm{mp} 174{ }^{\circ} \mathrm{C}\right)$; UV $\lambda_{\max }(\mathrm{EtOH})$ $393 \mathrm{~nm}$. IR $\left(\mathrm{cm}^{-1}\right)$ 2964, 2785, 1735, 1581. ${ }^{1} \mathrm{H}$ NMR $(500 \mathrm{MHz}$, DMSO- $\left.d_{6}\right) \delta 8.60(1 \mathrm{H}, \mathrm{s}, \mathrm{H}-8), 13.9(1 \mathrm{H}, \mathrm{s}, \mathrm{NH})$. LRMS $\left(\mathrm{ES}^{+}\right) \mathrm{m} / z$ $172.6[\mathrm{M}+\mathrm{H}]^{+}$.

2-Fluoro-9H-purine (11). ${ }^{48,49}$ To a stirred suspension of 6-chloro2-fluoropurine (10) $(0.30 \mathrm{~g}, 1.74 \mathrm{mmol})$ and palladium hydroxide on carbon $(20 \% \mathrm{w} / \mathrm{w}, 0.30 \mathrm{~g})$ in methanol $(15 \mathrm{~mL})$ was added ammonium formate $(0.34 \mathrm{~g}, 5.35 \mathrm{mmol})$. The suspension was heated under reflux for $1 \mathrm{~h}$ before filtering through a pad of Celite, eluting with methanol $(20 \mathrm{~mL})$. Removal of volatiles under reduced pressure afforded 11 as a white solid $(240 \mathrm{mg}, 100 \%) ; \mathrm{mp} 219^{\circ} \mathrm{C}$ (dec.) (lit., ${ }^{48}$ decomposed at $\left.216{ }^{\circ} \mathrm{C}\right) .{ }^{1} \mathrm{H}$ NMR $\left(300 \mathrm{MHz}\right.$, DMSO- $\left.d_{6}\right) \delta 8.60(1 \mathrm{H}$, s, H-8), 9.01 (1H, s, H-6), 13.9 (1H, s, NH). LRMS (ES $\left.{ }^{+}\right) m / z 139.2$ $[\mathrm{M}+\mathrm{H}]^{+}$.

Phenyl-(9H-purin-2-yl)amine (12). Prepared from aniline (0.18 $\mathrm{mL}, 2 \mathrm{mmol}$ ) and 2-fluoro- $9 \mathrm{H}$-purine (11, $0.138 \mathrm{~g}, 1.0 \mathrm{mmol}$ ) in accordance with general procedure $\mathrm{A}$. The reaction mixture was stirred under reflux for overnight. After removal of the solvent in vacuo, the residue was dissolved in EtOAc $(10-30 \mathrm{~mL})$, washed with saturated aqueous $\mathrm{NaHCO}_{3}(3 \times 30 \mathrm{~mL})$, and dried $\left(\mathrm{MgSO}_{4}\right)$. The crude material was purified by chromatography (silica; 0-20\% $\mathrm{MeOH}: \mathrm{DCM})$, followed by recrystallization from DCM to afford 12 as an off-white solid (65 mg, 31\%); $R_{\mathrm{f}} 0.20$ (5\% MeOH:DCM); mp 200-201 ${ }^{\circ} \mathrm{C}$; UV $\lambda_{\max }(\mathrm{EtOH}) 328,270,239,206 \mathrm{~nm}$. IR $\left(\mathrm{cm}^{-1}\right)$ $3234,3103,2804,1624,1602,1539,1402,1292,1217 .{ }^{1} \mathrm{H}$ NMR (300 MHz, DMSO- $\left.d_{6}\right) \delta 6.91\left(1 \mathrm{H}, \mathrm{t}, J=7.5 \mathrm{~Hz}, \mathrm{H}-4^{\prime}\right), 7.28(2 \mathrm{H}, \mathrm{t}, J=7.5$ $\mathrm{Hz}, \mathrm{H}-3^{\prime}$ and $\left.\mathrm{H}-5^{\prime}\right), 7.83\left(2 \mathrm{H}, \mathrm{d}, J=9.0 \mathrm{~Hz}, \mathrm{H}-2^{\prime}\right.$ and $\left.\mathrm{H}-6^{\prime}\right), 8.82(1 \mathrm{H}$, s, H-6), $8.24(1 \mathrm{H}, \mathrm{s}, \mathrm{H}-8), 9.53(1 \mathrm{H}, \mathrm{s}, \mathrm{NH}), 12.9(1 \mathrm{H}, \mathrm{s}, \mathrm{NH})$. LRMS $\left(\mathrm{ES}^{+}\right) \mathrm{m} / z 212.0[\mathrm{M}+\mathrm{H}]^{+}$. HRMS calcd for $\mathrm{C}_{11} \mathrm{H}_{10} \mathrm{~N}_{5}[\mathrm{M}+\mathrm{H}]^{+}$ 212.0931, found 212.0933 .

4-(9H-Purin-2-ylamino)benzenesulfonamide (13). Prepared following general procedure A from 2-fluoro- $9 \mathrm{H}$-purine $(11,0.10 \mathrm{~g}$, $0.725 \mathrm{mmol}$ ). Recrystallization from $\mathrm{MeOH}: \mathrm{H}_{2} \mathrm{O}$ gave 13 as a palewhite solid (74 mg, 35\%); $R_{\mathrm{f}} 0.40$ (20\% MeOH:DCM); mp >320 ${ }^{\circ} \mathrm{C}$ (dec); UV $\lambda_{\max }$ (EtOH) 287, $213 \mathrm{~nm}$. IR $\left(\mathrm{cm}^{-1}\right) 3223,1585,1481$, 1307, 1251, 1148, 1091. ${ }^{1} \mathrm{H}$ NMR $\left(300 \mathrm{MHz}\right.$, DMSO-d $\left.d_{6}\right) \delta 7.17(2 \mathrm{H}$, s, $\left.\mathrm{SO}_{2} \mathrm{NH}_{2}\right), 7.73\left(2 \mathrm{H}, \mathrm{d}, J=9.0 \mathrm{~Hz}, \mathrm{H}-3^{\prime}\right.$ and $\left.\mathrm{H}-5^{\prime}\right), 7.99(2 \mathrm{H}, \mathrm{d}, J=$ $9.0 \mathrm{~Hz}, \mathrm{H}-2^{\prime}$ and $\left.\mathrm{H}-6^{\prime}\right), 8.33(1 \mathrm{H}, \mathrm{s}, \mathrm{H}-6), 8.00(1 \mathrm{H}, \mathrm{s}, \mathrm{H}-8), 10.00$ $(1 \mathrm{H}, \mathrm{s}, \mathrm{NH})$. LRMS $\left(\mathrm{ES}^{+}\right) \mathrm{m} / z 291.0[\mathrm{M}+\mathrm{H}]^{+}$. HRMS calcd for $\mathrm{C}_{11} \mathrm{H}_{11} \mathrm{~N}_{6} \mathrm{O}_{2} \mathrm{~S}[\mathrm{M}+\mathrm{H}]^{+}$291.0659, found 291.0659.

$N^{2}$-Phenylguanine 2,2,2-trifluoroacetate (15). The title compound was prepared following general procedure A using 2-bromohypoxanthine $(14,1.00 \mathrm{~g}, 4.7 \mathrm{mmol})$ and aniline $(0.9 \mathrm{~mL}, 9.40 \mathrm{mmol})$ to yield 15 as a white solid $(1.17 \mathrm{~g}, 73 \%)$. The isolated compound was pure by analytical HPLC without the need for further purification; $\mathrm{mp}$ 229-231 ${ }^{\circ} \mathrm{C}$; UV $\lambda_{\max }(\mathrm{EtOH}) 273 \mathrm{~nm}$. IR $\left(\mathrm{cm}^{-1}\right) 3332,3128,2943$, $2756,2555,2387,1678,1572 .{ }^{1} \mathrm{H}$ NMR $\left(300 \mathrm{MHz}, \mathrm{DMSO}-d_{6}\right) \delta 7.07$ $\left(1 \mathrm{H}, \mathrm{t}, J=7.5 \mathrm{~Hz}, \mathrm{H}-4^{\prime}\right), 7.36\left(2 \mathrm{H}, \mathrm{dd}, J=7.5,8.0 \mathrm{~Hz}, \mathrm{H}-3^{\prime}\right.$ and $\left.\mathrm{H}-5^{\prime}\right)$, $7.62\left(2 \mathrm{H}, \mathrm{d}, J=8.0 \mathrm{~Hz}, \mathrm{H}-2^{\prime}\right.$ and $\left.\mathrm{H}-6^{\prime}\right), 7.94(1 \mathrm{H}, \mathrm{s}, \mathrm{H}-8), 8.46(1 \mathrm{H}$, br s, $\mathrm{NH}), 9.00(1 \mathrm{H}, \mathrm{br} \mathrm{s}, \mathrm{NH}) .{ }^{13} \mathrm{C}$ NMR $\left(75 \mathrm{MHz}, \mathrm{CDCl}_{3}\right) \delta 113$, $120,123,129,138,139,150,152,155$. LRMS $\left(\mathrm{ES}^{+}\right) \mathrm{m} / z 228.3[\mathrm{M}+$ $\mathrm{H}]^{+}$. HRMS calcd for $\mathrm{C}_{11} \mathrm{H}_{10} \mathrm{~N}_{5} \mathrm{O}[\mathrm{M}+\mathrm{H}]^{+}$228.0881, found 228.0880 .

6-Oxo-2-((4-sulfamoylphenyl)amino)-6,9-dihydro-1H-purine 2,2,2-trifluoroacetate (16). Synthesized in accordance with general procedure A from 2-bromohypoxanthine $(14,0.10 \mathrm{~g}, 0.47 \mathrm{mmol})$ to yield 16 as an amorphous white solid $(60 \mathrm{mg}, 42 \%)$; $\mathrm{mp}>300{ }^{\circ} \mathrm{C}$; UV $\lambda_{\max }(\mathrm{EtOH}) 275 \mathrm{~nm}$. IR $\left(\mathrm{cm}^{-1}\right) 3348,3113,2990,2877,2792,2333$, $1672,1575,1365,1153 .{ }^{1} \mathrm{H}$ NMR $\left(300 \mathrm{MHz}\right.$, DMSO-d $\left.d_{6}\right) \delta 7.28(2 \mathrm{H}$, s, $\left.\mathrm{SO}_{2} \mathrm{NH}_{2}\right), 7.79\left(4 \mathrm{H}, \mathrm{m}, \mathrm{H}-2^{\prime}, \mathrm{H}-3^{\prime}, \mathrm{H}-5^{\prime}\right.$ and $\left.\mathrm{H}-6^{\prime}\right), 8.31(1 \mathrm{H}, \mathrm{s}, \mathrm{H}-$ 8), $9.25(1 \mathrm{H}$, br s, $\mathrm{NH}), 10.84(1 \mathrm{H}$, br s, $\mathrm{NH}) .{ }^{13} \mathrm{C} \mathrm{NMR}(75 \mathrm{MHz}$,
$\left.\mathrm{CDCl}_{3}\right) \delta 113,120,123,127,138,139,150,152,156 . \mathrm{LRMS}\left(\mathrm{ES}^{+}\right)$ $m / z 307.3[\mathrm{M}+\mathrm{H}]^{+}$.

1-(2-Amino-9H-purin-6-yl)-4-aza-1-azoniabicyclo[2.2.2]octane (17). ${ }^{34}$ 1,4-Diazabicyclo[2.2.2] octane $(9.90 \mathrm{~g}, 88.4 \mathrm{mmol})$ was added to a solution of 2-amino-6-chloropurine $9(5.00 \mathrm{~g}, 29.5 \mathrm{mmol})$ in DMSO $(100 \mathrm{~mL})$ over $1 \mathrm{~h}$, and the mixture was stirred at room temperature for $24 \mathrm{~h}$. The resulting white precipitate was filtered and washed with diethyl ether. The solid was suspended in DCM (200 $\mathrm{mL}$ ) and stirred for $1 \mathrm{~h}$. After filtration and washing with DCM several times, 9 was obtained as a white solid, which was dried in vacuo (7.95 g, 96\%) and used without further purification; $\mathrm{mp} 230^{\circ} \mathrm{C}$ (dec) (lit., ${ }^{34}$ decomposed at $\left.230{ }^{\circ} \mathrm{C}\right) .{ }^{1} \mathrm{H}$ NMR $\left(300 \mathrm{MHz}, \mathrm{D}_{2} \mathrm{O}\right) \delta 3.31(6 \mathrm{H}, \mathrm{t}, J=$ $7.5 \mathrm{~Hz},\left(\mathrm{~N}\left(\mathrm{CH}_{2}\right)_{3}\right), 4.07\left(6 \mathrm{H}, \mathrm{t}, J=7.5 \mathrm{~Hz},\left(\mathrm{~N}^{+}\left(\mathrm{CH}_{2}\right)_{3}\right), 8.13(1 \mathrm{H}, \mathrm{s}\right.$, $\mathrm{H}-8) .{ }^{13} \mathrm{C}$ NMR $\left(75 \mathrm{MHz}, \mathrm{D}_{2} \mathrm{O}\right) \delta 38.7,53.4,116.0,143.7,151.3$, 158.4 .

General Procedure B. 2-Amino-6-chloropurine (9, $1.0 \mathrm{~mol}$ equiv) was added to a solution prepared from metallic sodium $(5.0 \mathrm{~mol}$ equiv) dissolved in the appropriate alcohol $(3.4 \mathrm{~mL} / \mathrm{mmol})$. The mixture was stirred at reflux until LCMS analysis indicated the absence of starting materials $(3-24 \mathrm{~h})$. After cooling, the reaction mixture was neutralized with glacial $\mathrm{AcOH}$ and the volatile material was removed in vacuo. Unless otherwise indicated, purification was achieved either by recrystallization from $\mathrm{H}_{2} \mathrm{O}$ or by adding $\mathrm{H}_{2} \mathrm{O}$ to the reaction mixture and extracting the product into EtOAc $(3 \times 100 \mathrm{~mL})$, followed by drying $\left(\mathrm{MgSO}_{4}\right)$ and removal of the solvent in vacuo.

General Procedure $C$. The appropriate alcohol ( $4.0 \mathrm{~mol}$ equiv) was added dropwise to a stirred suspension of $\mathrm{NaH}$ ( 3.0 mol equiv) in DMSO $(2.5-3.0 \mathrm{~mL} / \mathrm{mmol})$, and the resulting mixture was stirred for $1-2 \mathrm{~h}$. To this was added DABCO-purine (17, $1.0 \mathrm{~mol}$ equiv) or the appropriate haloheterocycle ( $1.0 \mathrm{~mol}$ equiv), and the mixture was stirred for $24 \mathrm{~h}$ with heating as specified. Water $(20-200 \mathrm{~mL})$ was added, and the basic emulsion was neutralized with glacial acetic acid. The aqueous phase was extracted with EtOAc $(3 \times 50-100 \mathrm{~mL})$, and the organic layers were washed with saturated aqueous $\mathrm{NaCl}(100$ $\mathrm{mL})$. The combined organic layers were dried $\left(\mathrm{MgSO}_{4}\right)$ and concentrated in vacuo to yield the crude product, which was purified by chromatography on silica and/or recrystallization from an appropriate solvent.

General Procedure D. To a stirred solution of hydrofluoroboric acid (50\%, aq, 20.0 mol equiv) cooled below $-20{ }^{\circ} \mathrm{C}$ was added the appropriate 2 -amino-6-alkoxypurine ( $1.0 \mathrm{~mol}$ equiv). While maintaining the temperature at $-15^{\circ} \mathrm{C}$, a solution of $\mathrm{NaNO}_{2}(2.0 \mathrm{~mol}$ equiv $)$ in $\mathrm{H}_{2} \mathrm{O}\left(1-3 \mathrm{~mL} / \mathrm{mmol} \mathrm{NaNO}_{2}\right)$ was added dropwise over $10 \mathrm{~min}$. The mixture was stirred at room temperature for $3 \mathrm{~h}$ and neutralized at $-15{ }^{\circ} \mathrm{C}$ by the dropwise addition of $15 \%\left(\mathrm{w} / \mathrm{v}\right.$ ) aqueous $\mathrm{Na}_{2} \mathrm{CO}_{3}$ solution, and the precipitated solid was collected by filtration and washed with $\mathrm{H}_{2} \mathrm{O}$. The residual solid was triturated with EtOAc $(3 \times$ $100 \mathrm{~mL}$ ) and filtered. The combined filtrates were concentrated under reduced pressure to furnish the product, which was purified as indicated.

General Procedure E. The appropriate 9-(tetrahydro-2H-pyran-2yl)-9H-purine ( $1.0 \mathrm{~mol}$ equiv) was dissolved in 2-propanol $(60 \mathrm{~mL} / \mathrm{g})$ and deionized water $(20 \mathrm{~mL} / \mathrm{g})$. Trifluoroacetic acid $(10.0 \mathrm{~mol}$ equiv) was added, and the reaction mixture was stirred at $100{ }^{\circ} \mathrm{C}$ for $2 \mathrm{~h}$ and cooled to room temperature, and the solution was adjusted to $\mathrm{pH} 8$ by addition of conc aqueous ammonia solution. The volume of solvent was reduced by $50 \%$ under reduced pressure; if a precipitate resulted, this was collected and washed with 2 -propanol $(2 \times 10 \mathrm{~mL})$. If precipitation was not observed following neutralization, the reaction mixture was partitioned between EtOAc $(20 \mathrm{~mL})$ and saturated aqueous $\mathrm{NaCl}$ solution $(20 \mathrm{~mL})$ The organic layer was dried $\left(\mathrm{Na}_{2} \mathrm{SO}_{4}\right)$ and evaporated under reduced pressure, and the product was purified by chromatography as specified.

General Procedure F. Tetrabutylammonium fluoride solution (1.0 $\mathrm{M}$ in THF, 1.5 mol equiv) was added dropwise to a stirred solution of the appropriate 6-triisopropylsilylethynylpurine (1.0 mol equiv) in anhydrous THF $(10-20 \mathrm{~mL})$, and the mixture was stirred for 5-15 min at ambient temperature under $\mathrm{N}_{2}$. Volatiles were evaporated under reduced pressure, and the residue was redissolved in EtOAc $(100 \mathrm{~mL} / \mathrm{g})$ and washed with saturated aqueous $\mathrm{NaCl}(100 \mathrm{~mL} / \mathrm{g})$, 
and the organic fraction was removed in vacuo. The product was purified as described.

General Procedure G. An oxygen-free solution of the required boronic acid or potassium trifluoroborate salt $(1.09 \mathrm{mmol})$, the appropriately 2 -substituted 6-chloro-9-(tetrahydro- $2 \mathrm{H}$-pyran-2-yl)-9Hpurine $(0.78 \mathrm{mmol})$, dicyclohexyl $\left(2^{\prime}, 6^{\prime}\right.$-dimethoxybiphenyl-2-yl $)$ phosphine (SPhos) $(2.5 \mathrm{~mol} \%)$, and palladium acetate $(1.0 \mathrm{~mol} \%)$ in toluene $(4 \mathrm{~mL})$ was degassed by bubbling nitrogen through the solution in a sealed vial for $5 \mathrm{~min}$. To the pale-yellow solution was added $\mathrm{K}_{3} \mathrm{PO}_{4}(1.56 \mathrm{mmol})$ and water (approximately $\left.50 \mu \mathrm{L}\right)$. The solution was again degassed for $15 \mathrm{~min}$ and subsequently heated to $100{ }^{\circ} \mathrm{C}$ for $18 \mathrm{~h}$. The black-brown suspension was filtered through Celite, eluting with $\mathrm{MeOH}(3 \times 10 \mathrm{~mL})$, and the product was isolated by chromatography as indicated.

2-Amino-6-ethoxypurine (18). ${ }^{50,51}$ Treatment of EtOH $(100 \mathrm{~mL})$ with $\mathrm{Na}(3.38 \mathrm{~g}, 147.5 \mathrm{mmol})$, followed by addition of 2-amino-6chloropurine 9 ( $5.0 \mathrm{~g}, 29.5 \mathrm{mmol}$ ) according to general procedure B, afforded the crude product. Recrystallization from $\mathrm{H}_{2} \mathrm{O}$ gave $\mathbf{1 8}$ as a white powder (4.75 g, 90\%); $R_{\mathrm{f}} 0.26(10 \% \mathrm{MeOH}: \mathrm{DCM}), \mathrm{mp} 247^{\circ} \mathrm{C}$ (dec) (lit. ${ }^{51} \mathrm{mp} 230{ }^{\circ} \mathrm{C}$ (dec), lit. ${ }^{50} \mathrm{mp} 293{ }^{\circ} \mathrm{C}(\mathrm{dec})$ ). IR $\left(\mathrm{cm}^{-1}\right)$ 3321, 2981, 2363, 2143, 1619, 1584, 1508, 1458, 1427, 1396, 1375, $1342,1285,1223,1161,1118 \mathrm{~cm}^{-1} .{ }^{1} \mathrm{H}$ NMR $\left(300 \mathrm{MHz}, \mathrm{DMSO}-d_{6}\right)$ $\delta 1.35\left(3 \mathrm{H}, \mathrm{t}, J=7.1 \mathrm{~Hz}, \mathrm{CH}_{3}\right), 4.44\left(2 \mathrm{H}, \mathrm{q}, J=7.1 \mathrm{~Hz}, \mathrm{CH}_{2}\right), 6.21$ $\left(2 \mathrm{H}, \mathrm{s}, \mathrm{NH}_{2}\right), 7.81(1 \mathrm{H}, \mathrm{s}, \mathrm{H}-8) .{ }^{13} \mathrm{C} \mathrm{NMR}\left(75 \mathrm{MHz}, \mathrm{DMSO}-d_{6}\right) \delta$ 14.9, 61.6, 160.1. LRMS $\left(\mathrm{ES}^{+}\right) \mathrm{m} / z 180.3[\mathrm{M}+\mathrm{H}]^{+}$. Anal. Found: C, 47.11; $\mathrm{H}, 4.97 ; \mathrm{N}, 38.97$. $\mathrm{C}_{7} \mathrm{H}_{9} \mathrm{~N}_{5} \mathrm{O}$ requires: $\mathrm{C}, 46.92 ; \mathrm{H}, 5.06 ; \mathrm{N}$, 39.09 .

2-Amino-6-n-propoxypurine (19). ${ }^{50,51}$ Treatment of 1-propanol $(20 \mathrm{~mL})$ with $\mathrm{Na}(0.68 \mathrm{~g}, 29.5 \mathrm{mmol})$, followed by addition of 2amino-6-chloropurine $9(1.0 \mathrm{~g}, 5.9 \mathrm{mmol})$ according to general procedure $\mathrm{B}$, gave the crude product. Recrystallization from $\mathrm{H}_{2} \mathrm{O}$ afforded $19(1.06 \mathrm{~g}, 93 \%)$ as a yellow solid, $R_{\mathrm{f}} 0.21(10 \%$ $\mathrm{MeOH}: \mathrm{DCM}$ ), mp $196-198{ }^{\circ} \mathrm{C}$ (lit. ${ }^{51} \mathrm{mp} 199-201{ }^{\circ} \mathrm{C}$, lit. ${ }^{50} \mathrm{mp}$ $\left.208^{\circ} \mathrm{C}\right)$. IR $\left(\mathrm{cm}^{-} 1\right) 3484,3389,3318,3203,2965,2935,2882,2522$, 2361, 2338, 1578, 1507, 1449, 1398, 1362, 1331, 1276, 1225, 1165, 1119. ${ }^{1} \mathrm{H}$ NMR (300 MHz, DMSO- $\left.d_{6}\right) \delta 0.97\left(3 \mathrm{H}, \mathrm{t}, J=7.4 \mathrm{~Hz}, \mathrm{CH}_{3}\right)$ $1.76\left(2 \mathrm{H}, \mathrm{tq}, J=7.2,7.2 \mathrm{~Hz}, \mathrm{CH}_{3} \mathrm{CH}_{2} \mathrm{CH}_{2} \mathrm{O}\right), 4.34(2 \mathrm{H}, \mathrm{t}, J=6.8 \mathrm{~Hz}$, $\left.\mathrm{OCH}_{2}\right), 6.20\left(2 \mathrm{H}, \mathrm{s}, \mathrm{NH}_{2}\right), 7.82(1 \mathrm{H}, \mathrm{s}, \mathrm{H}-8), 12.37(1 \mathrm{H}, \mathrm{br} \mathrm{s}, \mathrm{NH})$. ${ }^{13} \mathrm{C}$ NMR $\left(75 \mathrm{MHz}\right.$, DMSO- $\left.d_{6}\right) \delta 10.7,22.2,67.3,138.5,160.1,160.3$, 162.2. LRMS $\left(\mathrm{ES}^{+}\right) \mathrm{m} / z$ 194.17 $[\mathrm{M}+\mathrm{H}]^{+}$. Anal. Found: C, 46.81; $\mathrm{H}$, 5.95; $\mathrm{N}, 33.78 . \mathrm{C}_{8} \mathrm{H}_{11} \mathrm{~N}_{5} \mathrm{O} \cdot 0.7 \mathrm{H}_{2} \mathrm{O}$ requires: $\mathrm{C}, 46.69 ; \mathrm{H}, 6.07 ; \mathrm{N}$, 34.03.

2-Amino-6-isopropoxypurine (20). ${ }^{31}$ Treatment of 2-propanol $(0.85 \mathrm{~mL}, 14.2 \mathrm{mmol})$ with $\mathrm{NaH}(0.26 \mathrm{mg}, 10.7 \mathrm{mmol})$ in DMSO $(10 \mathrm{~mL})$, followed by addition of $\mathbf{1 7}(1.0 \mathrm{~g}, 3.6 \mathrm{mmol})$ was performed according to general procedure $\mathrm{C}$, to afford the crude product. Purification by chromatography (silica; 5-10\% MeOH:DCM) afforded 20 as a yellow oil. Trituration of the oil with diethyl ether afforded $20(0.38 \mathrm{~g}, 55 \%)$ as an off-white solid; $R_{\mathrm{f}} 0.28(10 \%$ MeOH:DCM), mp 205-207 ${ }^{\circ} \mathrm{C}$ (lit. $\left.{ }^{31} \mathrm{mp} \mathrm{209-210}{ }^{\circ} \mathrm{C}\right) . \mathrm{IR}\left(\mathrm{cm}^{-1}\right)$ 3491, 3331, 3195, 2976, 2773, 1618, 1580, 1504, 1445, 1391, 1372, $1321,1272,1224,1180,1144 .{ }^{1} \mathrm{H}$ NMR $\left(300 \mathrm{MHz}, \mathrm{DMSO}-d_{6}\right) \delta 1.33$ $\left(6 \mathrm{H}, \mathrm{d}, J=6.2 \mathrm{~Hz}, 2 \times \mathrm{CH}_{3}\right), 5.47(1 \mathrm{H}$, septet, $J=6.2 \mathrm{~Hz}, \mathrm{OCH}), 6.17$ $\left(2 \mathrm{H}, \mathrm{s}, \mathrm{NH}_{2}\right), 7.81(1 \mathrm{H}, \mathrm{s}, \mathrm{H}-8), 12.39(1 \mathrm{H}, \mathrm{br} \mathrm{s}, \mathrm{NH}) .{ }^{13} \mathrm{C}$ NMR $(75$ MHz, DMSO- $\left.d_{6}\right) \delta 22.3,68.2,160.1$. LRMS $\left(\mathrm{ES}^{+}\right) \mathrm{m} / z$ 194.15 $[\mathrm{M}+$ $\mathrm{H}]^{+}$. Anal. Found: C, 49.48; H, 5.64; N, 35.71. $\mathrm{C}_{8} \mathrm{H}_{11} \mathrm{~N}_{5} \mathrm{O} \cdot 0.1 \mathrm{H}_{2} \mathrm{O}$ requires: $\mathrm{C}, 49.27 ; \mathrm{H}, 5.79 ; \mathrm{N}, 35.91$

2-Amino-6-(2-methyl-1-propoxy)purine (21). ${ }^{31}$ 2-Methyl-1-propanol $(1.31 \mathrm{~mL}, 14.2 \mathrm{mmol})$ was added to $\mathrm{NaH}(0.25 \mathrm{mg}, 10.7 \mathrm{mmol})$ in DMSO $(10 \mathrm{~mL})$, followed by addition of $17(1.0 \mathrm{~g}, 3.6 \mathrm{mmol})$ according to general procedure $\mathrm{C}$, to afford the crude product. Trituration of the residual yellow oil with petrol furnished a cream solid. Purification by chromatography (silica; $5-10 \% \mathrm{MeOH}: \mathrm{DCM}$ ) gave $21(0.42 \mathrm{~g}, 57 \%)$ as pale-yellow crystals; $R_{\mathrm{f}} 0.41(10 \% \mathrm{MeOH} /$ DCM), mp $180-182^{\circ} \mathrm{C}$ (lit. $\left.{ }^{31} \mathrm{mp} \mathrm{89-92}{ }^{\circ} \mathrm{C}\right)$. IR $\left(\mathrm{cm}^{-1}\right) 3512,3473$, $3359,2958,2875,1620,1575,1507,1451,1386,1363,1332,1272$, $1223,1159,1121 .{ }^{1} \mathrm{H}$ NMR $\left(300 \mathrm{MHz}, \mathrm{DMSO}-d_{6}\right) \delta 0.98(6 \mathrm{H}, \mathrm{d}, J=$ $\left.6.7 \mathrm{~Hz}, 2 \times \mathrm{CH}_{3}\right), 2.07\left(1 \mathrm{H}\right.$, septet, $\left.J=6.7 \mathrm{~Hz}, \mathrm{OCH}_{2} \mathrm{CH}\right), 4.17(2 \mathrm{H}$, $\left.\mathrm{d}, J=6.8 \mathrm{~Hz}, \mathrm{OCH}_{2}\right), 6.21\left(2 \mathrm{H}, \mathrm{s}, \mathrm{NH}_{2}\right), 7.82(1 \mathrm{H}, \mathrm{s}, \mathrm{H}-8) .{ }^{13} \mathrm{C} \mathrm{NMR}$ $\left(75 \mathrm{MHz}, \mathrm{DMSO}-d_{6}\right) \delta 19.4,27.8,71.9,160.1,162.2 . \mathrm{LRMS}\left(\mathrm{ES}^{-}\right) \mathrm{m} /$ $z 206.81[\mathrm{M}-\mathrm{H}]^{-}$.

2-Amino-6-(1-methylpropoxy)purine (22). ${ }^{31}$ Treatment of 2butanol $(20 \mathrm{~mL})$ with $\mathrm{Na}(0.68 \mathrm{~g}, 29.5 \mathrm{mmol})$, followed by addition of 2-amino-6-chloropurine $9(1.0 \mathrm{~g}, 5.9 \mathrm{mmol})$ was performed according to general procedure $\mathrm{B}$, to give a pale-yellow solid. $\mathrm{H}_{2} \mathrm{O}$ was added, and the product was extracted into EtOAc $(3 \times 100 \mathrm{~mL})$, dried $\left(\mathrm{MgSO}_{4}\right)$, and the solvent removed in vacuo to yield $22(0.90 \mathrm{~g}, 74 \%)$ as off-white crystals, $R_{\mathrm{f}} 0.3(10 \% \mathrm{MeOH}: \mathrm{DCM}) ; \mathrm{mp} 77^{\circ} \mathrm{C}$ (dec) (lit. $\left.{ }^{31} \mathrm{mp} 88-90{ }^{\circ} \mathrm{C}\right)$. IR $\left(\mathrm{cm}^{-1}\right) 3322,3190,2971,2936,2876,2772$, $1616,1575,1504,1451,1393,1375,1326,1273,1222 .{ }^{1} \mathrm{H}$ NMR (300 $\left.\mathrm{MHz}, \mathrm{DMSO}-d_{6}\right) \delta 0.91\left(3 \mathrm{H}, \mathrm{t}, J=7.4 \mathrm{~Hz}, \mathrm{CH}_{3} \mathrm{CH}_{2} \mathrm{CHCH}_{3}\right), 1.29$ $\left(3 \mathrm{H}, \mathrm{d}, J=6.2 \mathrm{~Hz}, \mathrm{CH}_{3} \mathrm{CH}_{2} \mathrm{CHCH}_{3}\right), 1.57-1.79(2 \mathrm{H}, \mathrm{m}$, $\left.\mathrm{CH}_{3} \mathrm{CH}_{2} \mathrm{CHCH}_{3}\right), 5.27-5.38\left(1 \mathrm{H}, \mathrm{m}, \mathrm{CH}_{3} \mathrm{CH}_{2} \mathrm{CHCH}_{3}\right), 6.19(2 \mathrm{H}$, s, $\left.\mathrm{NH}_{2}\right), 7.79(1 \mathrm{H}, \mathrm{s}, \mathrm{H}-8) .{ }^{13} \mathrm{C}$ NMR $\left(75 \mathrm{MHz}, \mathrm{DMSO}-d_{6}\right) \delta 10.0$, 19.8, 28.9, 72.7, 137.7, 160.1. LRMS $\left(\mathrm{ES}^{-}\right) \mathrm{m} / z 206.08[\mathrm{M}-\mathrm{H}]^{-}$. Anal. Found: $\mathrm{C}, 51.52 ; \mathrm{H}, 6.43 ; \mathrm{N}, 32.64 . \mathrm{C}_{9} \mathrm{H}_{13} \mathrm{~N}_{5} \mathrm{O} \cdot 0.25 \mathrm{H}_{2} \mathrm{O}$ requires: $\mathrm{C}, 51.05 ; \mathrm{H}, 6.43 ; \mathrm{N}, 33.08$.

2-Amino-6-(prop-2-ynyloxy)purine (23). ${ }^{51}$ Treatment of propargyl alcohol $(0.83 \mathrm{~mL}, 14.2 \mathrm{mmol})$ with $\mathrm{NaH}(0.25 \mathrm{mg}, 10.7 \mathrm{mmol})$ in DMSO $(10 \mathrm{~mL})$, followed by addition of $17(1.0 \mathrm{~g}, 3.6 \mathrm{mmol})$ was performed according to general procedure $\mathrm{C}$, affording the product 23 $(0.54 \mathrm{~g}, 80 \%)$ as a beige solid, $R_{\mathrm{f}} 0.26(10 \% \mathrm{MeOH}: \mathrm{DCM}) ; \mathrm{mp} 181$ ${ }^{\circ} \mathrm{C}$ (dec) (lit. $\left.{ }^{51} \mathrm{mp} 230{ }^{\circ} \mathrm{C}(\mathrm{dec})\right)$. IR $\left(\mathrm{cm}^{-1}\right)$ 3319, 3190, 2777, 2126, $1651,1621,1589,1510,1460,1438,1400,1339,1277,1221,1184$, 1142. ${ }^{1} \mathrm{H}$ NMR $\left(300 \mathrm{MHz}, \mathrm{DMSO}-d_{6}\right) \delta 3.58(1 \mathrm{H}, \mathrm{t}, J=2.4 \mathrm{~Hz}$, $\left.\mathrm{OCH}_{2} \mathrm{CCH}\right), 5.10\left(2 \mathrm{H}, \mathrm{d}, J=2.4 \mathrm{~Hz}, \mathrm{OCH}_{2} \mathrm{CCH}\right), 6.35(2 \mathrm{H}, \mathrm{s}$, $\left.\mathrm{NH}_{2}\right), 7.85(1 \mathrm{H}, \mathrm{s}, \mathrm{H}-8) .{ }^{13} \mathrm{C}$ NMR $\left(75 \mathrm{MHz}, \mathrm{DMSO}-d_{6}\right) \delta 53.0,78.0$, 79.6, 113.7, 138.5, 155.7, 159.1, 159.9. LRMS $\left(\mathrm{ES}^{+}\right) \mathrm{m} / z 190.00[\mathrm{M}+$ $\mathrm{H}]^{+}$.

2-Fluoro-6-ethoxypurine (24). Prepared following general procedure D from $18(1.0 \mathrm{~g}, 5.6 \mathrm{mmol})$ to give 24 as a white solid $(0.22 \mathrm{~g}$, $22 \%) ; R_{\mathrm{f}} 0.39$ (10\% MeOH/DCM); mp $225-227{ }^{\circ} \mathrm{C}$. IR $\left(\mathrm{cm}^{-1}\right)$ $3117,2997,2740,2669,2506,2363,2339,1836,1760,1605,1487$, $1425,1373,1343,1281,1219,1151,1111,1038,1007 .{ }^{1} \mathrm{H}$ NMR (300 $\left.\mathrm{MHz}, \mathrm{DMSO}-d_{6}\right) \delta 1.41\left(3 \mathrm{H}, \mathrm{t}, J=7.1 \mathrm{~Hz}, \mathrm{CH}_{3}\right), 4.57(2 \mathrm{H}, \mathrm{q}, J=7.1$ $\left.\mathrm{Hz}, \mathrm{CH}_{2}\right), 8.39(1 \mathrm{H}, \mathrm{s}, \mathrm{H}-8), 13.55(1 \mathrm{H}, \mathrm{br} \mathrm{s}, \mathrm{NH}) .{ }^{13} \mathrm{C}$ NMR $(75$ $\left.\mathrm{MHz}, \mathrm{DMSO}-d_{6}\right) \delta 14.6\left(\mathrm{CH}_{3}\right), 64.0\left(\mathrm{CH}_{2}\right), 144.0\left(\mathrm{C}^{8}\right)$. LRMS $\left(\mathrm{ES}^{+}\right)$ $m / z 183.1[\mathrm{M}+\mathrm{H}]^{+}$. Anal. Found: $\mathrm{C}, 44.80 ; \mathrm{H}, 3.67 ; \mathrm{N}, 29.48$. $\mathrm{C}_{7} \mathrm{H}_{7} \mathrm{~N}_{4} \mathrm{OF} \cdot 0.3 \mathrm{H}_{2} \mathrm{O}$ requires: C, 44.83; $\mathrm{H}, 4.08 ; \mathrm{N}, 29.87$.

2-Fluoro-6-n-propoxypurine (25). Synthesized in accordance with general procedure D from $19(1.0 \mathrm{~g}, 5.2 \mathrm{mmol})$ to yield $25(0.58 \mathrm{~g}$, $57 \%$ ) as a white solid; $R_{\mathrm{f}} 0.18$ (70\% EtOAc:petrol); mp 197-199 ${ }^{\circ} \mathrm{C}$. IR $\left(\mathrm{cm}^{-1}\right) 3105,2973,2944,2886,2741,2680,2523,2362,2338$, 1772, 1607, 1422, 1368, 1347, 1269, 1213, 1149, 1038. ${ }^{1} \mathrm{H}$ NMR (300 $\left.\mathrm{MHz}, \mathrm{DMSO}-d_{6}\right) \delta 1.00\left(3 \mathrm{H}, \mathrm{t}, J=7.4 \mathrm{~Hz}, \mathrm{CH}_{3}\right) 1.82(2 \mathrm{H}, \mathrm{tq}, J=7.1$, $\left.7.1 \mathrm{~Hz}, \mathrm{CH}_{3} \mathrm{CH}_{2} \mathrm{CH}_{2} \mathrm{O}\right), 4.47\left(2 \mathrm{H}, \mathrm{t}, J=6.7 \mathrm{~Hz}, \mathrm{CH}_{3} \mathrm{CH}_{2} \mathrm{CH}_{2} \mathrm{O}\right)$, $8.39(1 \mathrm{H}, \mathrm{s}, \mathrm{H}-8), 13.55(1 \mathrm{H}, \mathrm{br} \mathrm{s}, \mathrm{NH}) .{ }^{13} \mathrm{C}$ NMR $(75 \mathrm{MHz}, \mathrm{DMSO}-$ $\left.d_{6}\right) \delta 10.5\left(\mathrm{CH}_{3}\right), 22.0\left(\mathrm{CH}_{3} \mathrm{CH}_{2} \mathrm{CH}_{2} \mathrm{O}\right), 69.4\left(\mathrm{O}^{6} \mathrm{CH}_{2}\right), 144.0(\mathrm{Ar}-$ C), $156.0\left(C^{8}\right), 158.8(\mathrm{Ar}-\mathrm{C})$. LRMS $\left(\mathrm{ES}^{+}\right) \mathrm{m} / z 154.0\left[\mathrm{M}-\mathrm{C}_{3} \mathrm{H}_{7}\right]^{+}$. HRMS calcd for $\mathrm{C}_{8} \mathrm{H}_{9} \mathrm{FN}_{4} \mathrm{O}\left[\mathrm{M}^{+}\right] 196.0760$, found 196.0757.

2-Fluoro-6-isopropoxypurine (26). Synthesized by general prodecure D from $20(0.30 \mathrm{~g}, 1.6 \mathrm{mmol})$ to yield 26 as a white solid $(0.16 \mathrm{~g}$, 53\%); $R_{\mathrm{f}} 0.22$ (70\% EtOAc:petrol); mp $184{ }^{\circ} \mathrm{C}$ (dec). IR $\left(\mathrm{cm}^{-1}\right)$ 2990, 2536, 1605, 1472, 1424, 1371, 1275, 1034, 1017. ${ }^{1} \mathrm{H}$ NMR (300 MHz, DMSO- $\left.d_{6}\right) \delta 1.40\left(6 \mathrm{H}, \mathrm{d}, J=6.2 \mathrm{~Hz}, 2 \times \mathrm{CH}_{3}\right), 5.49(1 \mathrm{H}$, septet, $J=6.2 \mathrm{~Hz}, \mathrm{OCH}), 8.37(1 \mathrm{H}, \mathrm{s}, \mathrm{H}-8), 13.52(1 \mathrm{H}, \mathrm{br} \mathrm{s}, \mathrm{NH}) .{ }^{13} \mathrm{C}$ NMR $\left(75 \mathrm{MHz}, \mathrm{DMSO}-d_{6}\right) \delta 22.0\left(\mathrm{CH}_{3}\right), 71.5\left(\mathrm{O}^{6} \mathrm{CH}\right), 162.2\left(\mathrm{C}^{8}\right)$. LRMS $\left(\mathrm{ES}^{+}\right) \mathrm{m} / z 154.0\left[\mathrm{M}-\mathrm{C}_{3} \mathrm{H}_{7}\right]^{+}$. HRMS calcd for $\mathrm{C}_{8} \mathrm{H}_{9} \mathrm{FN}_{4} \mathrm{O}$ $\left[\mathrm{M}^{+}\right]$196.0760, found 196.0760 .

2-Fluoro-6-(2-methyl-1-propoxy)purine (27). From 21 (0.40 g, 1.9 $\mathrm{mmol})$ in accordance with general procedure D to yield $27(0.25 \mathrm{~g}$, $62 \%$ ) as a white solid; $R_{\mathrm{f}} 0.25$ (70\% EtOAc:petrol); mp 202-204 ${ }^{\circ} \mathrm{C}$. IR $\left(\mathrm{cm}^{-1}\right) 3130,3059,2968,2937,2884,2804,2693,2528,1760$, $1700,1603,1476,1451,1418,1364,1341,1267,1211,1140,1113$, 1040. ${ }^{1} \mathrm{H}$ NMR (300 MHz, DMSO- $\left.d_{6}\right) \delta 1.01(6 \mathrm{H}, \mathrm{d}, J=6.7 \mathrm{~Hz}, 2 \times$ $\left.\mathrm{CH}_{3}\right), 2.13\left(1 \mathrm{H}\right.$, septet, $\left.J=6.7 \mathrm{~Hz}, \mathrm{OCH}_{2} \mathrm{CH}\right), 4.30(2 \mathrm{H}, \mathrm{d}, J=6.7$ $\left.\mathrm{Hz}, \mathrm{OCH}_{2}\right), 8.40(1 \mathrm{H}, \mathrm{s}, \mathrm{H}-8), 13.55(1 \mathrm{H}, \mathrm{br} \mathrm{s}, \mathrm{NH}) .{ }^{13} \mathrm{C}$ NMR $(75$ $\left.\mathrm{MHz}, \mathrm{DMSO}-d_{6}\right) \delta 19.2\left(\mathrm{CH}_{3}\right), 27.7\left(\mathrm{O}^{6} \mathrm{CH}_{2} \mathrm{CH}\right), 73.7\left(\mathrm{O}^{6} \mathrm{CH}_{2}\right)$. 
LRMS $\left(\mathrm{ES}^{+}\right) m / z 154.0\left[\mathrm{M}-\mathrm{C}_{3} \mathrm{H}_{7}\right]^{+}$. HRMS calcd for $\mathrm{C}_{9} \mathrm{H}_{11} \mathrm{FN}_{4} \mathrm{O}$ $\left[\mathrm{M}^{+}\right]$210.0917, found 210.0922 .

2-Fluoro-6-(1-methylpropoxy)purine (28). Prepared according to general procedure D from $22(0.66 \mathrm{~g}, 3.2 \mathrm{mmol})$ as a white solid $(0.29$ g, 43\%); $R_{\mathrm{f}} 0.21$ (70\% EtOAc:petrol); mp $187-189{ }^{\circ} \mathrm{C}$. IR $\left(\mathrm{cm}^{-1}\right)$ $2975,2940,1604,1468,1421,1372,1344,1277,1211,1148,1038 .{ }^{1} \mathrm{H}$ NMR $\left(300 \mathrm{MHz}\right.$, DMSO- $\left.d_{6}\right) \delta 0.93(3 \mathrm{H}, \mathrm{t}, J=7.4 \mathrm{~Hz}$, $\left.\mathrm{CH}_{3} \mathrm{CH}_{2} \mathrm{CHCH}_{3}\right), 1.36\left(3 \mathrm{H}, \mathrm{d}, J=6.2 \mathrm{~Hz}, \mathrm{CH}_{3} \mathrm{CH}_{2} \mathrm{CHCH}_{3}\right)$, 1.66-1.83 (2H, m, $\left.\mathrm{CH}_{3} \mathrm{CH}_{2} \mathrm{CHCH}_{3}\right), 5.29-5.37(1 \mathrm{H}, \mathrm{m}$, $\left.\mathrm{CH}_{3} \mathrm{CH}_{2} \mathrm{CHCH}_{3}\right), 8.37$ (1H, s, H-8), $13.55(1 \mathrm{H}, \mathrm{s}, \mathrm{NH}) .{ }^{13} \mathrm{C}$ NMR

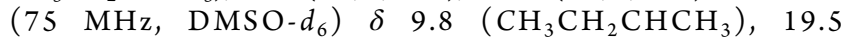
$\left(\mathrm{CH}_{3} \mathrm{CH}_{2} \mathrm{CHCH}_{3}\right), 28.6\left(\mathrm{CH}_{3} \mathrm{CH}_{2} \mathrm{CHCH}_{3}\right), 75.9\left(\mathrm{CH}_{3} \mathrm{CH}_{2} \mathrm{CHCH}_{3}\right)$, $158.8\left(\mathrm{C}^{8}\right)$. LRMS $\left(\mathrm{ES}^{-}\right) \mathrm{m} / z$ 209.1 $[\mathrm{M}-\mathrm{H}]^{-}$. Anal. Found: $\mathrm{C}$, 51.71; H, 5.27; N, 26.33. $\mathrm{C}_{9} \mathrm{H}_{11} \mathrm{~N}_{4} \mathrm{OF}$ requires: $\mathrm{C}, 51.42 ; \mathrm{H}, 5.27 ; \mathrm{N}$, 26.65 .

2-Fluoro-6-(prop-2-ynyloxy)purine (29). Synthesized from 23 $(0.28 \mathrm{~g}, 1.5 \mathrm{mmol})$ in accordance with general procedure $\mathrm{D}$ as a pale-yellow solid (77 mg, 27\%); $R_{\mathrm{f}} 0.18$ (70\% EtOAc:petrol); mp 201 ${ }^{\circ} \mathrm{C}$ (dec). IR $\left(\mathrm{cm}^{-1}\right) 3261,3123,2970,2864,2803,2363,2336,2140$, $1651,1607,1460,1379,1341,1275,1217,1142,1107,1048 .{ }^{1} \mathrm{H}$ NMR $\left(300 \mathrm{MHz}, \mathrm{DMSO}-d_{6}\right) \delta 3.70\left(1 \mathrm{H}, \mathrm{t}, J=2.4 \mathrm{~Hz}, \mathrm{OCH}_{2} \mathrm{CCH}\right), 5.23$ $\left(2 \mathrm{H}, \mathrm{d}, J=2.2 \mathrm{~Hz}, \mathrm{OCH}_{2} \mathrm{CCH}\right), 8.45(1 \mathrm{H}, \mathrm{s}, \mathrm{H}-8) .{ }^{13} \mathrm{C} \mathrm{NMR}(75$ $\left.\mathrm{MHz}, \mathrm{DMSO}-d_{6}\right) \delta 55.4\left(\mathrm{O}^{6} \mathrm{CH}_{2} \mathrm{CCH}\right), 78.5\left(\mathrm{O}^{6} \mathrm{CH}_{2} \mathrm{CCH}\right), 79.1$

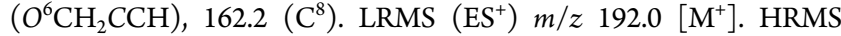
calcd for $\mathrm{C}_{8} \mathrm{H}_{5} \mathrm{FN}_{4} \mathrm{O}\left[\mathrm{M}^{+}\right]$192.0447, found 192.0448.

2-Sulfanilyl-6-ethoxypurine (30). Prepared from 24 (0.20 g, 1.1 $\mathrm{mmol}$ ) following general procedure A. Purification by chromatography (silica; 5-20\% MeOH:DCM) afforded $30(39 \mathrm{mg}, 11 \%)$ as an offwhite solid; $R_{\mathrm{f}} 0.20$ (10\% MeOH:DCM); mp $265^{\circ} \mathrm{C}$ (dec); UV $\lambda_{\max }$ (EtOH) 257, $241 \mathrm{~nm} . \mathrm{IR}\left(\mathrm{cm}^{-1}\right)$ 3473, 3317, 3223, 2984, 2787, 2363, $2338,1628,1578,1540,1499,1442,1376,1312,1254,1142,1013 .{ }^{1} \mathrm{H}$ NMR $\left(300 \mathrm{MHz}\right.$, DMSO- $\left.d_{6}\right) \delta 1.43\left(3 \mathrm{H}, \mathrm{t}, J=7.1 \mathrm{~Hz}, \mathrm{CH}_{3}\right), 4.59$ $\left(2 \mathrm{H}, \mathrm{q}, J=7.1 \mathrm{~Hz}, \mathrm{CH}_{2}\right), 7.16\left(2 \mathrm{H}, \mathrm{s}, \mathrm{NH}_{2}\right), 7.71(2 \mathrm{H}, \mathrm{d}, J=8.9 \mathrm{~Hz}$, $\mathrm{H}-3^{\prime}$ and $\left.\mathrm{H}-5^{\prime}\right), 7.97\left(2 \mathrm{H}, \mathrm{d}, J=8.9 \mathrm{~Hz}, \mathrm{H}-2^{\prime}\right.$ and $\left.\mathrm{H}-6^{\prime}\right), 8.08(1 \mathrm{H}, \mathrm{s}$, $\mathrm{H}-8), 9.76(1 \mathrm{H}, \mathrm{s}, \mathrm{NH}), 12.69(1 \mathrm{H}, \mathrm{s}, \mathrm{NH}) .{ }^{13} \mathrm{C} \mathrm{NMR}(75 \mathrm{MHz}$, DMSO- $\left.d_{6}\right) \delta 14.9\left(\mathrm{CH}_{3}\right), 62.6\left(\mathrm{CH}_{2}\right), 117.6(\mathrm{Ar}-\mathrm{C}), 126.8(\mathrm{Ar}-\mathrm{C})$, $155.1\left(\mathrm{C}^{8}\right)$. LRMS $\left(\mathrm{ES}^{+}\right) \mathrm{m} / z$ 335.0 $[\mathrm{M}+\mathrm{H}]^{+}$. HRMS calcd for $\mathrm{C}_{13} \mathrm{H}_{14} \mathrm{~N}_{6} \mathrm{O}_{3} \mathrm{~S}\left[\mathrm{M}^{+}\right]$334.0848, found 334.0835.

2-Sulfanilyl-6-propoxypurine (31). Prepared from 25 (0.25 g, 1.3 $\mathrm{mmol}$ ) according to general procedure A. Purification by chromatography (silica; $50-100 \%$ EtOAc:petrol followed by $0-10 \%$ $\mathrm{MeOH}: \mathrm{DCM})$, afforded $31(0.25 \mathrm{~g}, 56 \%)$ as a beige solid; $R_{\mathrm{f}} 0.10$ (70\% EtOAc:petrol); mp $207^{\circ} \mathrm{C}$ (dec); UV $\lambda_{\max }(\mathrm{EtOH}) 310 \mathrm{~nm}$. IR $\left(\mathrm{cm}^{-1}\right) 3251,3115,2971,2934,2878,2361,2340,1700,1596,1580$, 1528, 1498, 1453, 1389, 1321, 1306, 1243, 1146, 1094. ${ }^{1} \mathrm{H}$ NMR (300 $\left.\mathrm{MHz}, \mathrm{DMSO}-d_{6}\right) \delta 1.03\left(3 \mathrm{H}, \mathrm{t}, J=7.4 \mathrm{~Hz}, \mathrm{CH}_{3}\right) 1.85(2 \mathrm{H}, \mathrm{tq}, J=6.9$, $\left.6.9 \mathrm{~Hz}, \mathrm{CH}_{3} \mathrm{CH}_{2} \mathrm{CH}_{2} \mathrm{O}\right), 4.50\left(2 \mathrm{H}, \mathrm{t}, J=6.8 \mathrm{~Hz}, \mathrm{CH}_{3} \mathrm{CH}_{2} \mathrm{CH}_{2} \mathrm{O}\right)$, $7.17\left(2 \mathrm{H}, \mathrm{s}, \mathrm{NH}_{2}\right), 7.72\left(2 \mathrm{H}, \mathrm{d}, J=8.8 \mathrm{~Hz}, \mathrm{H}-2^{\prime}\right.$ and $\left.\mathrm{H}-6^{\prime}\right), 7.97(2 \mathrm{H}$, d, $J=8.8 \mathrm{~Hz}, \mathrm{H}-3^{\prime}$ and $\left.\mathrm{H}-5^{\prime}\right), 8.09(1 \mathrm{H}, \mathrm{s}, \mathrm{H}-8), 9.77(1 \mathrm{H}, \mathrm{s}, \mathrm{NH})$, $12.96(1 \mathrm{H}, \mathrm{br} \mathrm{s}, \mathrm{NH}) .{ }^{13} \mathrm{C}$ NMR $\left(75 \mathrm{MHz}, \mathrm{DMSO}-d_{6}\right) \delta 10.7\left(\mathrm{CH}_{3}\right)$, $22.2\left(\mathrm{CH}_{3} \mathrm{CH}_{2} \mathrm{CH}_{2} \mathrm{O}\right), 68.2\left(\mathrm{O}^{6} \mathrm{CH}_{2}\right), 117.6(\mathrm{CHCNH}), 126.8$ $\left(\mathrm{CHCSO}_{2} \mathrm{NH}_{2}\right), 135.7(\mathrm{Ar}-\mathrm{CNH}), 144.6\left(\mathrm{CSO}_{2} \mathrm{NH}_{2}\right), 155.1\left(\mathrm{C}^{8}\right)$. LRMS $\left(\mathrm{ES}^{+}\right) \mathrm{m} / z$ 349.8 $[\mathrm{M}+\mathrm{H}]^{+}$. HRMS calcd for $\mathrm{C}_{14} \mathrm{H}_{16} \mathrm{~N}_{6} \mathrm{O}_{3} \mathrm{~S}$ $\left[\mathrm{M}^{+}\right]$348.1005, found 348.0994; Anal. Found: C 45.41; H 4.75; N 22.40. $\mathrm{C}_{14} \mathrm{H}_{16} \mathrm{~N}_{6} \mathrm{O}_{3} \mathrm{~S} \cdot 0.3 \mathrm{CF}_{3} \mathrm{CO}_{2} \mathrm{H}$ requires: $\mathrm{C}, 45.83 ; \mathrm{H}, 4.29 ; \mathrm{N}$, 21.97.

2-Sulfanilyl-6-isopropoxypurine (32). Synthesized in accordance with general procedure A from $26(0.07 \mathrm{~g}, 0.4 \mathrm{mmol})$. Purification by chromatography (silica; 50-100\% EtOAc:petrol followed by $0-10 \%$ MeOH:DCM), afforded $32(38 \mathrm{mg}, 31 \%)$ as a beige solid; $R_{\mathrm{f}} 0.03$ (70\% EtOAc:petrol); mp $177^{\circ} \mathrm{C}$ (dec); UV $\lambda_{\max }(\mathrm{EtOH}) 307 \mathrm{~nm}$. IR $\left(\mathrm{cm}^{-1}\right) 3472,3312,3210,3117,2982,2793,2691,2643,2362,2342$, $1715,1685,1617,1597,1578,1538,1500,1440,1381,1306,1255$, $1140,1117,1096 .{ }^{1} \mathrm{H}$ NMR $\left(300 \mathrm{MHz}\right.$, DMSO- $\left.d_{6}\right) \delta 1.42(6 \mathrm{H}, \mathrm{d}, J=$ $\left.6.2 \mathrm{~Hz}, 2 \times \mathrm{CH}_{3}\right), 5.59(1 \mathrm{H}$, septet, $J=6.2 \mathrm{~Hz}, \mathrm{OCH}), 7.16(2 \mathrm{H}, \mathrm{s}$, $\left.\mathrm{NH}_{2}\right), 7.72\left(2 \mathrm{H}, \mathrm{d}, J=8.8 \mathrm{~Hz}, \mathrm{H}-2^{\prime}\right.$ and $\left.\mathrm{H}-6^{\prime}\right), 7.96(2 \mathrm{H}, \mathrm{d}, J=8.8 \mathrm{~Hz}$, $2 \times \mathrm{H}-3^{\prime}$ and $\left.\mathrm{H}-5^{\prime}\right), 8.07(1 \mathrm{H}, \mathrm{s}, \mathrm{H}-8), 9.74(1 \mathrm{H}, \mathrm{s}, \mathrm{NH}), 12.91(1 \mathrm{H}$, br s, NH). ${ }^{13} \mathrm{C}$ NMR $\left(75 \mathrm{MHz}, \mathrm{DMSO}-d_{6}\right) \delta 21.9\left(\mathrm{CH}_{3}\right), 69.0$ $\left(\mathrm{O}^{6} \mathrm{CH}\right), 117.1(\mathrm{CHCNH}), 126.5\left(\mathrm{CHCSO}_{2} \mathrm{NH}_{2}\right), 135.2(\mathrm{Ar}-\mathrm{CNH})$,
$144.3\left(\mathrm{CSO}_{2} \mathrm{NH}_{2}\right), 154.7\left(\mathrm{C}^{8}\right)$. LRMS $\left(\mathrm{ES}^{+}\right) \mathrm{m} / z 349.8[\mathrm{M}+\mathrm{H}]^{+}$. HRMS calcd for $\mathrm{C}_{14} \mathrm{H}_{16} \mathrm{~N}_{6} \mathrm{O}_{3} \mathrm{~S}\left[\mathrm{M}^{+}\right] 348.1005$, found 348.1007.

2-Sulfanilyl-6-(2-methyl-1-propoxy)purine (33). Prepared following general procedure A from $27(0.12 \mathrm{~g}, 0.6 \mathrm{mmol})$. Purification by chromatography (silica, $50-100 \%$ EtOAc:petrol followed by $0-10 \%$ $\mathrm{MeOH}: \mathrm{DCM})$, afforded $33(0.11 \mathrm{~g}, 53 \%)$ as a light-brown solid; $R_{\mathrm{f}}$ 0.21 (70\% EtOAc:petrol); mp $133{ }^{\circ} \mathrm{C}$ (dec); UV $\lambda_{\max }(\mathrm{EtOH}) 308$ $\mathrm{nm}$. IR $\left(\mathrm{cm}^{-1}\right) 3285,3108,2962,2360,2337,1595,1576,1543,1499$, 1438, 1396, 1321, 1249, 1221, 1144, 1096, 1016. ${ }^{1} \mathrm{H}$ NMR (300 MHz, DMSO- $\left.d_{6}\right) \delta 1.03\left(6 \mathrm{H}, \mathrm{d}, J=6.7 \mathrm{~Hz}, 2 \times \mathrm{CH}_{3}\right), 2.16(1 \mathrm{H}$, septet, $J=$ $\left.6.7 \mathrm{~Hz}, \mathrm{OCH}_{2} \mathrm{CH}\right), 4.33\left(2 \mathrm{H}, \mathrm{d}, J=6.8 \mathrm{~Hz}, \mathrm{OCH}_{2} \mathrm{CH}\right), 7.17(2 \mathrm{H}, \mathrm{s}$, $\left.\mathrm{NH}_{2}\right), 7.71\left(2 \mathrm{H}, \mathrm{d}, J=8.9 \mathrm{~Hz}, \mathrm{H}-2^{\prime}\right.$ and $\left.\mathrm{H}-6^{\prime}\right), 7.97(2 \mathrm{H}, \mathrm{d}, J=8.9 \mathrm{~Hz}$, $\mathrm{H}-3^{\prime}$ and $\left.\mathrm{H}-5^{\prime}\right), 8.09(1 \mathrm{H}, \mathrm{s}, \mathrm{H}-8), 9.77(1 \mathrm{H}, \mathrm{s}, \mathrm{NH}) .{ }^{13} \mathrm{C}$ NMR $(75$ MHz, DMSO-d $\left.d_{6}\right) \delta 19.4\left(\mathrm{CH}_{3}\right), 27.9\left(\mathrm{O}^{6} \mathrm{CH}_{2} \mathrm{CH}\right), 72.7\left(\mathrm{O}^{6} \mathrm{CH}_{2}\right)$, $117.6(\mathrm{CHCNH}), 126.8\left(\mathrm{CHCSO}_{2} \mathrm{NH}_{2}\right), 135.7(\mathrm{Ar}-\mathrm{CNH}), 144.6$ $\left(\mathrm{CSO}_{2} \mathrm{NH}_{2}\right), 155.1\left(\mathrm{C}^{8}\right)$. LRMS $\left(\mathrm{ES}^{+}\right) \mathrm{m} / z 363.8[\mathrm{M}+\mathrm{H}]^{+}$. HRMS calcd for $\mathrm{C}_{15} \mathrm{H}_{18} \mathrm{~N}_{6} \mathrm{O}_{3} \mathrm{~S}\left[\mathrm{M}^{+}\right]$362.1161, found 362.1145 .

(R/S)-6-(1-Methylpropoxy)-2-sulfanilylpurine (34). Synthesized from $28(0.1 \mathrm{~g}, 0.5 \mathrm{mmol})$ following general procedure A. Purification by chromatography (silica; $50-100 \%$ EtOAc:petrol followed by $0-$ 10\% MeOH:DCM), afforded 34 (41 mg, 24\%) as a light-brown solid; $R_{\mathrm{f}} 0.10$ (70\% EtOAc:petrol); mp $162{ }^{\circ} \mathrm{C}$ (dec); UV (EtOH) $\lambda_{\max } 308$ nm. IR $\left(\mathrm{cm}^{-1}\right) 3649,3317,3219,3106,2972,2938,2874,2363,2340$, $1701,1596,1579,1528,1498,1453,1389,1306,1243,1145,1094 .{ }^{1} \mathrm{H}$ NMR $\left(300 \mathrm{MHz}\right.$, DMSO- $\left.d_{6}\right) \delta 0.97(3 \mathrm{H}, \mathrm{t}, J=7.4 \mathrm{~Hz}$, $\left.\mathrm{CH}_{3} \mathrm{CH}_{2} \mathrm{CHCH}_{3}\right), 1.39\left(3 \mathrm{H}, \mathrm{d}, J=6.2 \mathrm{~Hz}, \mathrm{CH}_{3} \mathrm{CH}_{2} \mathrm{CHCH}_{3}\right)$, $1.68-1.87\left(2 \mathrm{H}, \mathrm{m}, \mathrm{CH}_{3} \mathrm{CH}_{2} \mathrm{CHCH}_{3}\right), 5.40-5.46(1 \mathrm{H}, \mathrm{m}$, $\left.\mathrm{CH}_{3} \mathrm{CH}_{2} \mathrm{CHCH}_{3}\right), 7.17\left(2 \mathrm{H}, \mathrm{s}, \mathrm{NH}_{2}\right), 7.72\left(2 \mathrm{H}, \mathrm{d}, J=8.8 \mathrm{~Hz}, \mathrm{H}-2^{\prime}\right.$ and $\left.\mathrm{H}-6^{\prime}\right), 7.96\left(2 \mathrm{H}, \mathrm{d}, J=8.9 \mathrm{~Hz}, \mathrm{H}-3^{\prime}\right.$ and $\left.\mathrm{H}-5^{\prime}\right), 8.13(1 \mathrm{H}, \mathrm{s}, \mathrm{H}-8)$, $9.76(1 \mathrm{H}, \quad \mathrm{s}, \mathrm{NH}) .{ }^{13} \mathrm{C}$ NMR $\left(75 \mathrm{MHz}, \mathrm{DMSO}-d_{6}\right) \delta 10.0$ $\left(\mathrm{CH}_{3} \mathrm{CH}_{2} \mathrm{CHCH}_{3}\right), 19.7\left(\mathrm{CH}_{3} \mathrm{CH}_{2} \mathrm{CHCH}_{3}\right), 28.8\left(\mathrm{CH}_{3} \mathrm{CH}_{2} \mathrm{CHCH}_{3}\right)$, $74.2\left(\mathrm{CH}_{3} \mathrm{CH}_{2} \mathrm{CHCH}_{3}\right), 117.6(\mathrm{CHCNH}), 126.8\left(\mathrm{CHCSO}_{2} \mathrm{NH}_{2}\right)$, $135.8(\mathrm{Ar}-\mathrm{CNH}), 144.6\left(\mathrm{CSO}_{2} \mathrm{NH}_{2}\right), 155.2\left(\mathrm{C}^{8}\right)$. LRMS $\left(\mathrm{ES}^{+}\right) \mathrm{m} / z$ $363.8[\mathrm{M}+\mathrm{H}]^{+}$. HRMS calcd for $\mathrm{C}_{15} \mathrm{H}_{18} \mathrm{~N}_{6} \mathrm{O}_{3} \mathrm{~S}\left[\mathrm{M}^{+}\right] 362.1161$, found 362.1166. Anal. Found: C, 48.32; H, 5.15; N, 22.08. $\mathrm{C}_{15} \mathrm{H}_{18} \mathrm{~N}_{6} \mathrm{O}_{3} S$. $0.2 \mathrm{CF}_{3} \mathrm{CO}_{2} \mathrm{H}$ requires: $\mathrm{C}, 48.02 ; \mathrm{H}, 4.76 ; \mathrm{N}, 21.82$.

2-Sulfanilyl-6-(prop-2-ynyloxy)purine (35). Synthesized in accordance with general procedure A from $29(0.045 \mathrm{~g}, 0.2 \mathrm{mmol})$. Purification by chromatography (silica; $50-100 \%$ EtOAc:petrol followed by $0-10 \% \mathrm{MeOH}: \mathrm{DCM})$, yielded $35(27 \mathrm{mg}, 34 \%)$ as a beige solid; $R_{\mathrm{f}} 0.1$ (70\% EtOAc:petrol); $\mathrm{mp} 161{ }^{\circ} \mathrm{C}$ (dec); UV (EtOH) $\lambda_{\max } 307 \mathrm{~nm}$. IR $\left(\mathrm{cm}^{-1}\right) 3370,3245,2971,2795,2361,2340$, $1715,1699,1619,1585,1575,1540,1499,1472,1454,1443,1396$, $1321,1307,1271,1143,1095 .{ }^{1} \mathrm{H}$ NMR $\left(300 \mathrm{MHz}, \mathrm{DMSO}-d_{6}\right) \delta 3.68$ $\left(1 \mathrm{H}, \mathrm{t}, J=2.3 \mathrm{~Hz}, \mathrm{OCH}_{2} \mathrm{CCH}\right), 5.24\left(2 \mathrm{H}, \mathrm{d}, J=2.3 \mathrm{~Hz}, \mathrm{OCH}_{2} \mathrm{CCH}\right)$, $7.18\left(2 \mathrm{H}, \mathrm{s}, \mathrm{NH}_{2}\right), 7.72\left(2 \mathrm{H}, \mathrm{d}, J=8.8 \mathrm{~Hz}, \mathrm{H}-2^{\prime}\right.$ and $\left.\mathrm{H}-6^{\prime}\right), 7.98(2 \mathrm{H}$, $\mathrm{d}, J=8.8 \mathrm{~Hz}, \mathrm{H}-3^{\prime}$ and $\left.\mathrm{H}-5^{\prime}\right), 8.13(1 \mathrm{H}, \mathrm{s}, \mathrm{H}-8), 9.87(1 \mathrm{H}, \mathrm{s}, \mathrm{NH}) .{ }^{13} \mathrm{C}$ NMR $\left.(75 \mathrm{MHz}, \text { DMSO-d })_{6}\right) \quad \delta 3.6\left(\mathrm{O}^{6} \mathrm{CH}_{2} \mathrm{CCH}\right), \quad 78.0$ $\left(\mathrm{O}^{6} \mathrm{CH}_{2} \mathrm{CCH}\right), 79.1\left(\mathrm{O}^{6} \mathrm{CH}_{2} \mathrm{CCH}\right), 115.1$ (Ar-C), $117.5(\mathrm{CHCNH})$, $126.5\left(\mathrm{CHCSO}_{2} \mathrm{NH}_{2}\right), 135.5(\mathrm{Ar}-\mathrm{CNH}), 139.9\left(\mathrm{C}^{2} \mathrm{NH}\right), 143.9$ $\left(\mathrm{CSO}_{2} \mathrm{NH}_{2}\right), 154.3\left(\mathrm{C}^{8}\right), 158.6(\mathrm{Ar}-\mathrm{C})$. LRMS $\left(\mathrm{ES}^{+}\right) \mathrm{m} / z$ 345.8 [M $+\mathrm{H}]^{+}$. HRMS calcd for $\mathrm{C}_{14} \mathrm{H}_{12} \mathrm{~N}_{6} \mathrm{O}_{3} \mathrm{~S}\left[\mathrm{M}^{+}\right]$344.0692, found 344.0700 .

6-Chloro-2-fluoro-9-(tetrahydro-2H-pyran-2-yl)-9H-purine (36). 3,4-Dihydropyran $(60 \mu \mathrm{L}, 0.58 \mathrm{mmol})$ was added dropwise over 10 min to a vigorously stirred solution of $10(0.10 \mathrm{~g}, 0.58 \mathrm{mmol})$ and (rac)-camphorsulfonic acid $(5 \mathrm{mg}, 0.02 \mathrm{mmol})$ in EtOAc $(50 \mathrm{~mL})$ at $65{ }^{\circ} \mathrm{C}$. The temperature was maintained at $65{ }^{\circ} \mathrm{C}$ for $18 \mathrm{~h}$. The resulting bright-yellow solution was neutralized to $\mathrm{pH} 7$ by careful addition of aqueous $\mathrm{NH}_{3}$ solution until a cloudy suspension persisted. The crude mixture was washed with brine $(2 \times 30 \mathrm{~mL})$ and the aqueous phase was re-extracted with EtOAc $(2 \times 30 \mathrm{~mL})$. The combined organic extracts were dried $\left(\mathrm{Na}_{2} \mathrm{SO}_{4}\right)$ and purified by chromatography (silica; 30\% EtOAc:petrol) to afford $\mathbf{3 6}$ as a paleyellow oil which became a white waxy solid on refrigeration $(110 \mathrm{mg}$, 75\%); mp 92-94 ${ }^{\circ} \mathrm{C}$; UV $\lambda_{\max }(\mathrm{EtOH}) 269 \mathrm{~nm}$. IR $\left(\mathrm{cm}^{-1}\right) 3132$, $2955,2876,1574 .{ }^{1} \mathrm{H}$ NMR $\left(500 \mathrm{MHz}\right.$, DMSO-d $\left.d_{6}\right) \delta 1.57-1.64(2 \mathrm{H}$, $\left.\mathrm{m}, \mathrm{CH}_{2}\right), 1.70-1.80(1 \mathrm{H}, \mathrm{m}, \mathrm{CH}), 1.94-2.02\left(2 \mathrm{H}, \mathrm{m}, \mathrm{CH}_{2}\right), 2.23-$ $2.32(1 \mathrm{H}, \mathrm{m}, \mathrm{CH}), 3.70-3.77(1 \mathrm{H}, \mathrm{m}, \mathrm{CH}), 4.00-4.06(1 \mathrm{H}, \mathrm{m}, \mathrm{CH})$, $5.71(1 \mathrm{H}, \mathrm{dd}, J=2.3,10.9 \mathrm{~Hz}, \mathrm{NCH}), 8.92(1 \mathrm{H}, \mathrm{s}, \mathrm{H}-8) .{ }^{13} \mathrm{C}$ NMR 
$\left(125 \mathrm{MHz}, \mathrm{DMSO}-d_{6}\right) \delta 22.0\left(N^{9}-\mathrm{CHCH}_{2} \mathrm{CH}_{2}\right), 24.3 \quad\left(N^{9}-\right.$ $\left.\mathrm{CHOCH}_{2} \mathrm{CH}_{2}\right), 29.6\left(\mathrm{~N}^{9}-\mathrm{CHCH}_{2} \mathrm{CH}_{2}\right), 67.7\left(\mathrm{~N}^{9}-\mathrm{CHOCH}_{2} \mathrm{CH}_{2}\right)$, $81.7\left(N^{9}-\mathrm{CH}\right), 130.1\left(\mathrm{~d}, J_{\mathrm{CF}}=4.8 \mathrm{~Hz}, \mathrm{Ar}-\mathrm{C}\right), 146.4\left(\mathrm{~d}, J_{\mathrm{CF}}=2.8 \mathrm{~Hz}\right.$, $\operatorname{Ar}-\mathrm{C}), 150.6\left(\mathrm{~d}, J_{\mathrm{CF}}=18.5 \mathrm{~Hz}, \mathrm{Ar}-\mathrm{C}\right), 153.2\left(\mathrm{~d}, J_{\mathrm{CF}}=17.6 \mathrm{~Hz}, \mathrm{Ar}-\mathrm{C}\right)$, $156.2\left(\mathrm{~d}, J_{\mathrm{CF}}=213.7 \mathrm{~Hz}, \mathrm{Ar}-\mathrm{C}^{2}\right)$. LRMS $\left(\mathrm{ES}^{+}\right) \mathrm{m} / z 257.2[\mathrm{M}+\mathrm{H}]^{+}$. 2-Fluoro-9-(tetrahydro-2H-pyran-2-yl)-6-((triisopropylsilyl)ethynyl)-9H-purine (37). An oxygen-free solution of $36(0.050 \mathrm{~g}, 1.95$ mmol), bis(triphenylphosphine)palladium(II) chloride (41 mg, 3.0 mol \%) and copper iodide (7 mg, $2.0 \mathrm{~mol} \%)$ in THF $(10 \mathrm{~mL})$ was degassed by bubbling nitrogen through the solution in a sealed vial for $5 \mathrm{~min}$. Triisopropylsilylacetylene $(0.50 \mathrm{~mL}, 2.2 \mathrm{mmol})$ and triethylamine $(0.70 \mathrm{~mL}, 4.9 \mathrm{mmol})$ were added to the mixture, which was again degassed for $15 \mathrm{~min}$. After stirring at room temperature for $18 \mathrm{~h}$, the black-brown suspension was filtered through Celite, eluting with $\mathrm{MeOH}(3 \times 20 \mathrm{~mL})$. Purification by chromatography (silica; $10 \%$ EtOAc:petrol) furnished 37 as a viscous yellow oil (78 mg, 99\%); $R_{\mathrm{f}}$ 0.30 (10\% EtOAc:petrol); UV $\lambda_{\max }(\mathrm{EtOH}) 303 \mathrm{~nm}$. IR $\left(\mathrm{cm}^{-1}\right) 3433$, 2946, 2866, 2706, 2361, 1703. ${ }^{1} \mathrm{H}$ NMR $\left(500 \mathrm{MHz}, \mathrm{DMSO}-d_{6}\right) \delta$ $1.12-1.25\left(21 \mathrm{H}, \mathrm{m}, \mathrm{Si}\left(\mathrm{CH}\left(\mathrm{CH}_{3}\right)_{2}\right)_{3}\right), 1.56-1.63\left(2 \mathrm{H}, \mathrm{m}, \mathrm{CH}_{2}\right)$, $1.71-1.80(1 \mathrm{H}, \mathrm{m}, \mathrm{CH}), 1.94-2.03\left(2 \mathrm{H}, \mathrm{m}, \mathrm{CH}_{2}\right), 2.20-2.30(1 \mathrm{H}, \mathrm{m}$, $\mathrm{CH}), 3.70-3.78(1 \mathrm{H}, \mathrm{m}, \mathrm{CH}), 4.01-4.07(1 \mathrm{H}, \mathrm{m}, \mathrm{CH}), 5.70(1 \mathrm{H}, \mathrm{dd}$, $J=2.2,10.8 \mathrm{~Hz}, \mathrm{NCH}), 8.89(1 \mathrm{H}, \mathrm{s}, \mathrm{H}-8) .{ }^{13} \mathrm{C} \mathrm{NMR}(125 \mathrm{MHz}$, DMSO-d $\left.d_{6}\right) \delta 10.5\left(\mathrm{Si}\left(\mathrm{CH}\left(\mathrm{CH}_{3}\right)_{2}\right)_{3}\right), 18.4\left(\mathrm{Si}\left(\mathrm{CH}\left(\mathrm{CH}_{3}\right)_{2}\right)_{3}\right), 22.0$ $\left(N^{9}-\mathrm{CHCH}_{2} \mathrm{CH}_{2}\right), 24.4\left(N^{9}-\mathrm{CHOCH}_{2} \mathrm{CH}_{2}\right), 29.7\left(N^{9}-\mathrm{CHCH}_{2} \mathrm{CH}_{2}\right)$, $67.7\left(N^{9}-\mathrm{CHOCH}_{2} \mathrm{CH}_{2}\right), 81.4\left(N^{9}-\mathrm{CH}\right), 100.2(\mathrm{C} \equiv \mathrm{C}), 102.8(\mathrm{C} \equiv$ C), $133.9\left(\mathrm{~d}, J_{\mathrm{CF}}=4.6 \mathrm{~Hz}, \mathrm{Ar}-\mathrm{C}\right), 140.8\left(\mathrm{~d}, J_{\mathrm{CF}}=17.9 \mathrm{~Hz}, \mathrm{Ar}-\mathrm{C}\right)$, $146.6\left(\mathrm{~d}, J_{\mathrm{CF}}=2.8 \mathrm{~Hz}, \mathrm{Ar}-\mathrm{C}\right), 153.8\left(\mathrm{~d}, J_{\mathrm{CF}}=17.8 \mathrm{~Hz}, \mathrm{Ar}-\mathrm{C}\right), 157.4$ (d, $\left.J_{\mathrm{CF}}=210.6 \mathrm{~Hz}, \mathrm{Ar}-\mathrm{C}^{2}\right)$. HRMS calcd for $\mathrm{C}_{21} \mathrm{H}_{32} \mathrm{FN}_{4} \mathrm{OSi}[\mathrm{M}+\mathrm{H}]^{+}$ 403.2324, found 403.2324.

2-Fluoro-6-((triisopropylsilyl)ethynyl)-9H-purine (38). The title compound was prepared according to general procedure $\mathrm{E}$ from 37 $(0.29 \mathrm{~g}, 0.72 \mathrm{mmol})$. Purification by chromatography (silica; $30 \%$ EtOAc:petrol) gave 38 as a pale-yellow oil (22 mg, $96 \%) ; R_{\mathrm{f}} 0.25(30 \%$ EtOAc:petrol); UV $\lambda_{\max }(\mathrm{EtOH}) 302 \mathrm{~nm}$. IR $\left(\mathrm{cm}^{-1}\right) 2945,2866$, $2361,2000,1584 .{ }^{1} \mathrm{H}$ NMR $\left(500 \mathrm{MHz}\right.$, DMSO- $\left.d_{6}\right) \delta 1.12-1.21(21 \mathrm{H}$, $\left.\mathrm{m}, \mathrm{Si}\left(\mathrm{CH}\left(\mathrm{CH}_{3}\right)_{2}\right)_{3}\right), 8.68(1 \mathrm{H}, \mathrm{s}, \mathrm{H}-8), 13.89(1 \mathrm{H}, \mathrm{br}, \mathrm{NH}) .{ }^{13} \mathrm{C}$ NMR $\left(125 \mathrm{MHz}, \mathrm{CDCl}_{3}\right) \delta 11.1\left(\mathrm{Si}\left(\mathrm{CH}\left(\mathrm{CH}_{3}\right)_{2}\right)_{3}\right), 18.5\left(\mathrm{Si}\left(\mathrm{CH}\left(\mathrm{CH}_{3}\right)_{2}\right)_{3}\right)$, 99.5 (C三C), 106.1 ( $\mathrm{C} \equiv \mathrm{C}), 131.2$ (m, Ar-C), 141.6 (m, Ar-C), 145.3 (m, Ar-C), 156.6 (m, Ar-C), 158.6 (d, $\left.J_{\mathrm{CF}}=217.4 \mathrm{~Hz}, \mathrm{Ar}-\mathrm{C}^{2}\right)$. HRMS calcd for $\mathrm{C}_{16} \mathrm{H}_{24} \mathrm{FN}_{4} \mathrm{Si}[\mathrm{M}+\mathrm{H}]^{+}$319.1749, found 319.1752.

6-(2-(Triisopropylsilyl)ethynyl)- $N$-phenyl-9H-purin-2-amine (39). Prepared in accordance with general procedure A from 38 (0.455 g, $1.43 \mathrm{mmol}$ ). Purified by chromatography (silica; $5 \% \mathrm{MeOH}: \mathrm{DCM}$ ) to give the title compound as a yellow gum $(0.39 \mathrm{~g}, 1.00 \mathrm{mmol}, 70 \%) ; R_{\mathrm{f}}$ 0.39 (5\% MeOH:DCM); UV $\lambda_{\max }(\mathrm{EtOH}) 276 \mathrm{~nm}$. IR $\left(\mathrm{cm}^{-1}\right) 3389$, 2361, 2021. ${ }^{1} \mathrm{H}$ NMR (500 MHz, $\left.\mathrm{CDCl}_{3}\right) \delta 1.09-1.22(21 \mathrm{H}, \mathrm{m}$, $\left.\mathrm{Si}\left(\mathrm{CH}\left(\mathrm{CH}_{3}\right)_{2}\right)_{3}\right), 7.00-7.04\left(1 \mathrm{H}, \mathrm{m}, \mathrm{H}-4^{\prime}\right), 7.22(1 \mathrm{H}, \mathrm{s}, \mathrm{NH}), 7.29$ $\left(2 \mathrm{H}, \mathrm{dd}, J=7.7,8.1 \mathrm{~Hz}, \mathrm{H}-3^{\prime}\right.$ and $\left.\mathrm{H}-5^{\prime}\right), 7.46(1 \mathrm{H}, \mathrm{s}, \mathrm{H}-8), 7.52(2 \mathrm{H}$, dd, $J=1.7,7.7 \mathrm{~Hz}, \mathrm{H}-2^{\prime}$ and $\left.\mathrm{H}-6^{\prime}\right), 10.42(1 \mathrm{H}, \mathrm{s}, \mathrm{NH}) .{ }^{13} \mathrm{C}$ NMR $(125$ $\left.\mathrm{MHz}, \mathrm{CDCl}_{3}\right) \delta 11.3\left(\mathrm{Si}\left(\mathrm{CH}\left(\mathrm{CH}_{3}\right)_{2}\right)_{3}\right), 18.7\left(\mathrm{Si}\left(\mathrm{CH}\left(\mathrm{CH}_{3}\right)_{2}\right)_{3}\right), 100.8$ $(\mathrm{C} \equiv \mathrm{C}), 101.8(\mathrm{C} \equiv \mathrm{C}), 120.7$ (Ar-C), 123.7 (Ar-C), 129.4 (Ar-C), 139.2 (Ar-C), 141.0 (Ar-C), 142.6 (Ar-C), 153.4 (Ar-C), 156.5 (ArC). LRMS $\left(\mathrm{ES}^{+}\right) \mathrm{m} / z 392.0[\mathrm{M}+\mathrm{H}]^{+}$.

4-(6-((Triisopropylsilyl)ethynyl)-9H-purin-2-ylamino)benzenesulfonamide (40). Following general procedure A employing $38(0.156 \mathrm{~g}, 0.49 \mathrm{mmol})$. Purification by reversed phase column chromatography (C18 silica; $25-95 \% \mathrm{MeCN} /$ water $+0.1 \%$ $\mathrm{HCOOH})$, afforded 40 as a yellow solid (70 mg, 30\%); mp 163$165^{\circ} \mathrm{C}$; UV $\lambda_{\max }(\mathrm{EtOH}) 361 \mathrm{~nm}$. IR $\left(\mathrm{cm}^{-1}\right) 3327,2944,2867,1569$, $1531,1368,1149 .{ }^{1} \mathrm{H}$ NMR (500 MHz, DMSO- $\left.d_{6}\right) \delta 1.15(21 \mathrm{H}, \mathrm{m}$, $\left.\mathrm{Si}\left(\mathrm{CH}\left(\mathrm{CH}_{3}\right)_{2}\right)_{3}\right), 7.17\left(2 \mathrm{H}, \mathrm{s}, \mathrm{SO}_{2} \mathrm{NH}_{2}\right), 7.70\left(2 \mathrm{H}, \mathrm{d}, J=9.0 \mathrm{~Hz}, \mathrm{H}-2^{\prime}\right.$ and $\left.\mathrm{H}-6^{\prime}\right), 7.95\left(2 \mathrm{H}, \mathrm{d}, J=9.0 \mathrm{~Hz}, \mathrm{H}-3^{\prime}\right.$ and $\left.\mathrm{H}-5^{\prime}\right), 8.33(1 \mathrm{H}, \mathrm{s}, \mathrm{H}-8)$. ${ }^{13} \mathrm{C}$ NMR $\left(125 \mathrm{MHz}\right.$, DMSO- $\left.d_{6}\right) \delta 10.6,18.4,90.6,98.4,117.1,123.4$, $126.5,135.6,144.0,155.5,165.0,166.3,166.5,291.0,286.5,215.5$. LRMS $\left(\mathrm{ES}^{+}\right) \mathrm{m} / z$ 471.4 $[\mathrm{M}+\mathrm{H}]^{+}$. HRMS calcd for $\mathrm{C}_{22} \mathrm{H}_{30} \mathrm{~N}_{6} \mathrm{O}_{2} \mathrm{SSi}$ $[\mathrm{M}+\mathrm{H}]^{+}$471.1987, found 471.1942.

6-Ethynyl-N-phenyl-9H-purin-2-amine (41). Treatment of 39 $(0.330 \mathrm{~g}, 0.84 \mathrm{mmol})$ according to general procedure $\mathrm{F}$, followed by purification by chromatography (silica; EtOAc), yielded 41 as a yellow solid (201 mg, 100\%); $R_{\mathrm{f}} 0.27$ (100\% EtOAc); mp 140-160 ${ }^{\circ} \mathrm{C}$ (dec);
UV $\lambda_{\max }$ (EtOH) $243 \mathrm{~nm} . \mathrm{IR}\left(\mathrm{cm}^{-1}\right) 3414,3111,3072,2920,2110$, 1704. ${ }^{1} \mathrm{H}$ NMR (500 MHz, DMSO- $\left.d_{6}\right) \delta 4.86(1 \mathrm{H}, \mathrm{s}, \mathrm{C} \equiv \mathrm{CH}), 6.91-$ $6.96\left(1 \mathrm{H}, \mathrm{m}, \mathrm{H}-4^{\prime}\right), 7.29\left(2 \mathrm{H}, \mathrm{dd}, J=7.6,8.0 \mathrm{~Hz}, \mathrm{H}-3^{\prime}\right.$ and $\left.\mathrm{H}-5^{\prime}\right), 7.80$ $\left(2 \mathrm{H}, \mathrm{dd}, J=2.0,8.0 \mathrm{~Hz}, \mathrm{H}-2^{\prime}\right.$ and $\left.\mathrm{H}-6^{\prime}\right), 8.30(1 \mathrm{H}, \mathrm{s}, \mathrm{H}-8), 9.70(1 \mathrm{H}$, s, NH), $13.17(1 \mathrm{H}, \mathrm{br}, \mathrm{NH}) .{ }^{13} \mathrm{C}$ NMR $\left(125 \mathrm{MHz}, \mathrm{DMSO}-d_{6}\right) \delta 87.0$ $(\mathrm{C} \equiv \mathrm{CH}), 118.3$ (Ar-C), 120.0 (Ar-C), 128.4 (Ar-C), 140.8 (Ar-C), 156.2 (Ar-C). HRMS calcd for $\mathrm{C}_{13} \mathrm{H}_{10} \mathrm{~N}_{5}[\mathrm{M}+\mathrm{H}]^{+}$236.0931, found 236.0934 .

4-(6-Ethynyl-9H-purin-2-ylamino)benzenesulfonamide (42). Deprotection of $40(0.050 \mathrm{~g}, 0.11 \mathrm{mmol})$ in accordance with general procedure $\mathrm{F}$, followed by purification by chromatography (silica; $10 \%$ $\mathrm{MeOH}: \mathrm{DCM})$, afforded $\mathbf{4 2}$ as a beige solid (18 mg, 50\%); mp 156$158{ }^{\circ} \mathrm{C}$. IR $\left(\mathrm{cm}^{-1}\right) 3347,3255,2920,2848,2118,1568,1529,1477$, 1128. UV $\lambda_{\max }(\mathrm{EtOH}) 356,292,215 \mathrm{~nm} .{ }^{1} \mathrm{H}$ NMR $(500 \mathrm{MHz}$, DMSO- $\left.d_{6}\right) \delta 4.90(1 \mathrm{H}, \mathrm{s}, \mathrm{C} \equiv \mathrm{CH}), 7.16\left(2 \mathrm{H}, \mathrm{s}, \mathrm{SO}_{2} \mathrm{NH}_{2}\right), 7.17(2 \mathrm{H}$, d, $J=9.0 \mathrm{~Hz}, \mathrm{H}-2^{\prime}$ and $\left.\mathrm{H}-6^{\prime}\right), 7.93\left(2 \mathrm{H}, \mathrm{d}, J=9.0 \mathrm{~Hz}, \mathrm{H}-3^{\prime}\right.$ and $\left.\mathrm{H}-5^{\prime}\right)$, $8.36(1 \mathrm{H}, \mathrm{s}, \mathrm{H}-8)$. LRMS $\left(\mathrm{ES}^{+}\right) \mathrm{m} / z 315.1[\mathrm{M}+\mathrm{H}]^{+}$. HRMS calcd for $\mathrm{C}_{13} \mathrm{H}_{10} \mathrm{~N}_{6} \mathrm{O}_{2} \mathrm{~S}[\mathrm{M}+\mathrm{H}]^{+}$315.0664, found 315.0687. Note: Insufficient material for ${ }^{13} \mathrm{C}$ NMR analysis.

6-Ethynyl-2-fluoro-9-(tetrahydro-2H-pyran-2-yl)-9H-purine (43). Deprotection of $37(68 \mathrm{mg}, 0.169 \mathrm{mmol})$ in accordance with general procedure $\mathrm{F}$, but using $1.2 \mathrm{~mol}$ equiv of $\mathrm{TBAF}$, followed by purification by chromatography (silica; 15-100\% EtOAc:petrol), afforded 43 as an off-white solid (41 mg, 100\%); $R_{\mathrm{f}} 0.32(50 \%$ EtOAc:petrol). ${ }^{1} \mathrm{H}$ NMR $\left(500 \mathrm{MHz}, \mathrm{CDCl}_{3}\right) \delta 1.58-1.63(1 \mathrm{H}, \mathrm{m}$, $\mathrm{CH}), 1.63-1.78\left(2 \mathrm{H}, \mathrm{m}, \mathrm{CH}_{2}\right), 1.90-1.99(1 \mathrm{H}, \mathrm{m}, \mathrm{CH}), 1.99-2.06$ $(1 \mathrm{H}, \mathrm{m}, \mathrm{CH}), 2.07-2.12(1 \mathrm{H}, \mathrm{m}, \mathrm{CH}), 3.68-3.74(1 \mathrm{H}, \mathrm{m}, \mathrm{CH}), 3.71$ $(1 \mathrm{H}, \mathrm{s}, \mathrm{C} \equiv \mathrm{CH}), 4.09-4.14(1 \mathrm{H}, \mathrm{m}, \mathrm{CH}), 5.65(1 \mathrm{H}, \mathrm{dd}, J=2.6,10.8$ $\mathrm{Hz}, \mathrm{NCH}), 8.26(1 \mathrm{H}, \mathrm{s}, \mathrm{H}-8)$. LRMS $\left(\mathrm{ES}^{+}\right) \mathrm{m} / z 247.0[\mathrm{M}+\mathrm{H}]^{+}$. Note: Insufficient material for ${ }^{13} \mathrm{C}$ NMR analysis.

6-Chloro-N-phenyl-9H-purin-2-amine (44). A mixture of $\mathrm{N}^{2}$ phenylguanine trifluoroacetate $(15)(2.00 \mathrm{~g}, 5.87 \mathrm{mmol})$ and $\mathrm{N}, \mathrm{N}$ diethylaniline $(1.9 \mathrm{~mL}, 11.73 \mathrm{mmol})$ in $\mathrm{POCl}_{3}(30 \mathrm{~mL})$ was heated at $115{ }^{\circ} \mathrm{C}$ for $60 \mathrm{~min}$. The resultant yellow solution was carefully added dropwise to crushed ice-water with stirring, and the aqueous solution was neutralized by cautious addition of aqueous $\mathrm{NaOH}$ solution (1.0 $\mathrm{M})$. The aqueous mixture was extracted with EtOAc $(2 \times 20 \mathrm{~mL})$, and the combined organic extracts were dried $\left(\mathrm{Na}_{2} \mathrm{SO}_{4}\right)$ and evaporated under reduced pressure. Purification by chromatography (silica; 50\% EtOAc:petrol) gave 44 as a white powder $(670 \mathrm{mg}, 46 \%)$; mp $172-$ $174{ }^{\circ} \mathrm{C}$; UV $\lambda_{\max }(\mathrm{EtOH}) 329,272 \mathrm{~nm} . \mathrm{IR}\left(\mathrm{cm}^{-1}\right) 3399,3289,1627$, $1601,1571,1540 .{ }^{1} \mathrm{H}$ NMR $\left(500 \mathrm{MHz}, \mathrm{DMSO}-d_{6}\right) \delta 6.89-6.92(1 \mathrm{H}$, $\left.\mathrm{t}, J=7.5 \mathrm{~Hz}, \mathrm{H}-4^{\prime}\right), 7.23-7.26\left(2 \mathrm{H}, \mathrm{dd}, J=7.4,7.5 \mathrm{~Hz}, \mathrm{H}-3^{\prime}\right.$ and $\mathrm{H}-$ $\left.5^{\prime}\right), 7.71-7.73\left(2 \mathrm{H}, \mathrm{d}, J=7.4 \mathrm{~Hz}, \mathrm{H}-2^{\prime}\right.$ and $\left.\mathrm{H}-6^{\prime}\right), 8.20(1 \mathrm{H}, \mathrm{s}, \mathrm{H}-8)$, $9.81\left(1 \mathrm{H}\right.$, br s, NH), $13.20\left(1 \mathrm{H}\right.$, br s, NH). ${ }^{13} \mathrm{C}$ NMR $(125 \mathrm{MHz}$, DMSO- $\left.d_{6}\right) \delta 118.6,121.5,128.5,140.3,142.4,155.5$. LRMS $\left(\mathrm{ES}^{+}\right) \mathrm{m} /$ $z 246.1[\mathrm{M}+\mathrm{H}]^{+}$.

6-Chloro-N-phenyl-9-(tetrahydro-2H-pyran-2-yl)-9H-purin-2amine (45). To a solution of $44(0.2 \mathrm{~g}, 0.82 \mathrm{mmol})$ and CSA (0.01 g, $0.041 \mathrm{mmol})$ in EtOAc $(25 \mathrm{~mL})$ was added DHP $(83 \mu \mathrm{L}, 0.90 \mathrm{mmol})$, and the mixture was heated at $75{ }^{\circ} \mathrm{C}$ for $17 \mathrm{~h}$. After cooling, the $\mathrm{pH}$ was adjusted to 8-9 with concentrated aqueous ammonia, and the volatiles were removed in vacuo. Purification by chromatography (silica; $1 / 2$ to $2 / 1$ EtOAc:petrol) furnished 45 as a waxy solid (194 mg, 72\%); mp 194-196 ${ }^{\circ} \mathrm{C}$; UV $\lambda_{\max }$ (EtOH) $330,275 \mathrm{~nm}$. IR $\left(\mathrm{cm}^{-1}\right)$ 3274, 3106, 2934, 2849, 1579, 1573, 1535. ${ }^{1} \mathrm{H}$ NMR (500 MHz, DMSO- $\left.d_{6}\right) \delta 1.38-1.39\left(2 \mathrm{H}, \mathrm{m}, \mathrm{CH}_{2}\right), 1.49-1.54(1 \mathrm{H}, \mathrm{m}, \mathrm{CH})$, $1.76-1.80\left(2 \mathrm{H}, \mathrm{m}, \mathrm{CH}_{2}\right), 2.09-2.17(1 \mathrm{H}, \mathrm{m}, \mathrm{CH}), 3.43-3.48(1 \mathrm{H}, \mathrm{m}$, $\mathrm{CH}), 3.81-3.83(1 \mathrm{H}, \mathrm{m}, \mathrm{CH}), 5.38-5.40(1 \mathrm{H}, \mathrm{dd}, J=2.0,11.0 \mathrm{~Hz}$, $\mathrm{NCH}), 6.74-6.77\left(1 \mathrm{H}, \mathrm{t}, J=8.5 \mathrm{~Hz}, \mathrm{H}-4^{\prime}\right), 7.07-7.11(2 \mathrm{H}, \mathrm{dd}, J=$ $7.5,8.5 \mathrm{~Hz}, \mathrm{H}-3^{\prime}$ and $\left.\mathrm{H}-5^{\prime}\right), 7.56-7.58\left(2 \mathrm{H}, \mathrm{d}, J=7.5 \mathrm{~Hz}, \mathrm{H}-2^{\prime}\right.$ and $\mathrm{H}-$ $\left.6^{\prime}\right), 8.26(1 \mathrm{H}, \mathrm{s}, \mathrm{H}-8), 9.79(1 \mathrm{H}, \mathrm{br} \mathrm{s}, \mathrm{NH}), 13.21(1 \mathrm{H}, \mathrm{br} \mathrm{s}, \mathrm{NH}) .{ }^{13} \mathrm{C}$ NMR $\left(125 \mathrm{MHz}, \mathrm{DMSO}-d_{6}\right) \delta 24.5,29.4,54.9,67.7,81.6,118.7$, 121.7, 124.7, 128.6, 140.1, 142.4, 149.3, 152.7, 155.3. LRMS $\left(\mathrm{ES}^{+}\right) \mathrm{m} /$ $z 329.7[\mathrm{M}+\mathrm{H}]^{+}$.

N-Phenyl-6-(prop-1-yn-1-yl)-9-(tetrahydro-2H-pyran-2-yl)-9Hpurin-2-amine (46). A suspension of $45(0.1 \mathrm{~g}, 0.30 \mathrm{mmol})$, $\mathrm{PdCl}_{2}\left(\mathrm{CH}_{3} \mathrm{CN}\right)_{2} \quad(0.8 \mathrm{mg}, 0.003 \mathrm{mmol})$, dicyclohexylphosphino$2^{\prime}, 4^{\prime}, 6^{\prime}$-triisopropylbiphenyl $(4.3 \mathrm{mg}, 0.009 \mathrm{mmol})$, and $\mathrm{Cs}_{2} \mathrm{CO}_{3}$ $(0.26 \mathrm{~g}, 0.79 \mathrm{mmol})$ in acetonitrile $(1 \mathrm{~mL})$ was degassed for $10 \mathrm{~min}$ 
under a nitrogen atmosphere at room temperature. While degassing, an excess of methylacetylene was condensed and added to the reaction mixture via a cannula, and the reaction mixture was heated and stirred at $40{ }^{\circ} \mathrm{C}$ for $2.5 \mathrm{~h}$. After cooling to room temperature, water $(5 \mathrm{~mL})$ was added and the mixture was extracted with EtOAc $(3 \times 15 \mathrm{~mL})$. The combined organic extracts were washed with brine $(10 \mathrm{~mL})$, dried $\left(\mathrm{MgSO}_{4}\right)$, and the solvent was evaporated under reduced pressure. Purification by chromatography (silica; $30 \%-80 \%$ EtOAc:petrol) gave 46 as a colorless glassy compound $(78 \mathrm{mg}, 77 \%) ; R_{\mathrm{f}} 0.27(30 \%$ EtOAc:petrol). ${ }^{1} \mathrm{H}$ NMR $\left(500 \mathrm{MHz}, \mathrm{CDCl}_{3}\right) \delta 1.62-1.85(3 \mathrm{H}, \mathrm{m}$, $\mathrm{CH}_{2}$ and $\left.\mathrm{CH}\right), 2.06-2.15\left(3 \mathrm{H}, \mathrm{m}, \mathrm{CH}_{2}\right.$ and $\left.\mathrm{CH}\right), 2.23(3 \mathrm{H}, \mathrm{s}, \mathrm{C} \equiv$ $\left.\mathrm{CCH}_{3}\right), 3.74-3.81(1 \mathrm{H}, \mathrm{m}, \mathrm{CH}), 4.15-4.21(1 \mathrm{H}, \mathrm{m}, \mathrm{CH}), 5.60-5.67$ $(1 \mathrm{H}, \mathrm{m}, \mathrm{CH}), 7.04\left(1 \mathrm{H}, \mathrm{dd}, J=7.4,7.3 \mathrm{~Hz}, \mathrm{H}-4^{\prime}\right), 7.34(2 \mathrm{H}, \mathrm{dd}, J=$ 8.5, 7.4 Hz, H-2' and $\left.\mathrm{H}-6^{\prime}\right), 7.47(1 \mathrm{H}, \mathrm{s}, \mathrm{NH}), 7.34(2 \mathrm{H}, \mathrm{dd}, J=8.6$, $0.9 \mathrm{~Hz}, \mathrm{H}-3^{\prime}$ and $\left.\mathrm{H}-5^{\prime}\right), 8.03$ (1H, s, H-8). LRMS $\left(\mathrm{ES}^{+}\right) m / z 334.0[\mathrm{M}$ $+\mathrm{H}]^{+}$. Note: Insufficient material for ${ }^{13} \mathrm{C}$ NMR analysis.

$\mathrm{N}$-Phenyl-6-(phenylethynyl)-9-(tetrahydro-2H-pyran-2-yl)-9Hpurin-2-amine (47). To a degassed suspension of $45(0.1 \mathrm{~g}, 0.30$ $\mathrm{mmol}), \mathrm{PdCl}_{2}\left(\mathrm{CH}_{3} \mathrm{CN}\right)_{2}(0.8 \mathrm{mg}, 0.003 \mathrm{mmol})$, dicyclohexylphosphino-2', $4^{\prime}, 6^{\prime}$-triisopropylbiphenyl $(4.3 \mathrm{mg}, 0.009 \mathrm{mmol})$, and $\mathrm{Cs}_{2} \mathrm{CO}_{3}$ $(0.26 \mathrm{~g}, 0.79 \mathrm{mmol})$ in anhydrous acetonitrile $(1 \mathrm{~mL})$ was added an excess of phenylacetylene, and the mixture was heated at $80^{\circ} \mathrm{C}$ for $2 \mathrm{~h}$. After cooling, water $(5 \mathrm{~mL})$ was added and the suspension was extracted with EtOAc $(3 \times 15 \mathrm{~mL})$. The combined organic extract was washed with brine $(10 \mathrm{~mL})$ and dried $\left(\mathrm{MgSO}_{4}\right)$, and the solvent was removed in vacuo. Purification by chromatography (silica; $0-80 \%$ EtOAc:petrol) gave 47 as a colorless glassy compound (94 mg, 78\%); $R_{\mathrm{f}} 0.53$ (65\% EtOAc:petrol). ${ }^{1} \mathrm{H}$ NMR $\left(500 \mathrm{MHz}, \mathrm{CDCl}_{3}\right) \delta 1.64-$ $1.88\left(3 \mathrm{H}, \mathrm{m}, \mathrm{CH}_{2}\right.$ and $\left.\mathrm{CH}\right), 2.07-2.19\left(3 \mathrm{H}, \mathrm{m}, \mathrm{CH}_{2}\right.$ and $\left.\mathrm{CH}\right), 3.74-$ $3.84(1 \mathrm{H}, \mathrm{m}, \mathrm{CH}), 4.16-4.24(1 \mathrm{H}, \mathrm{m}, \mathrm{CH}), 5.63-5.71(1 \mathrm{H}, \mathrm{m}, \mathrm{CH})$, 7.03-7.08 (1H, m, H-4'), 7.32-7.46 (5H, m, phenyl), $7.50(1 \mathrm{H}, \mathrm{s}$, $\mathrm{NH}), 7.70\left(2 \mathrm{H}, \mathrm{d}, J=8.3 \mathrm{~Hz}, \mathrm{H}-2^{\prime}\right.$ and $\left.\mathrm{H}-6^{\prime}\right), 7.72-7.75\left(2 \mathrm{H}, \mathrm{m}, \mathrm{H}-3^{\prime}\right.$ and $\left.\mathrm{H}-5^{\prime}\right), 8.08(1 \mathrm{H}, \mathrm{s}, \mathrm{H}-8) .{ }^{13} \mathrm{C} \mathrm{NMR}\left(125 \mathrm{MHz}, \mathrm{CDCl}_{3}\right) \delta 22.9$, 25.0, 31.5, 68.8, 82.2, 84.1, 97.6, 118.9, 121.5, 122.5, 128.5, 129.0, 129.4, 130.0, 132.9, 139.8, 141.0, 142.3, 152.5, 156.2. LRMS $\left(\mathrm{ES}^{+}\right) \mathrm{m} /$ $z 396.4[\mathrm{M}+\mathrm{H}]^{+}$.

N-Phenyl-6-(prop-1-yn-1-yl)-9H-purin-2-amine (48). Prepared in accordance with general procedure $\mathrm{E}$ from $46(0.061 \mathrm{~g}, 0.18 \mathrm{mmol})$. Recrystallization from EtOAc-petrol gave $\mathbf{4 8}$ as a pale-yellow solid (18 mg, 40\%); mp $155-158{ }^{\circ} \mathrm{C}$ (dec); UV $\lambda_{\max }(\mathrm{EtOH}) 272 \mathrm{~nm}$. IR $\left(\mathrm{cm}^{-1}\right) 1529,1577,2236,2919 .{ }^{1} \mathrm{H}$ NMR $\left(500 \mathrm{MHz}, \mathrm{MeOD}-d_{4}\right) \delta$ $2.12\left(3 \mathrm{H}, \mathrm{s}, \mathrm{C} \equiv \mathrm{CCH}_{3}\right), 6.87\left(1 \mathrm{H}, \mathrm{t}, J=7.3 \mathrm{~Hz}, \mathrm{H}-4^{\prime}\right), 7.18(2 \mathrm{H}, \mathrm{t}, J=$ $7.8 \mathrm{~Hz}, \mathrm{H}-2^{\prime}$ and $\left.\mathrm{H}-6^{\prime}\right), 7.63\left(2 \mathrm{H}, \mathrm{d}, J=7.9 \mathrm{~Hz}, \mathrm{H}-3^{\prime}\right.$ and $\left.\mathrm{H}-5^{\prime}\right), 8.04$ $(1 \mathrm{H}, \mathrm{s}, \mathrm{H}-8) .{ }^{13} \mathrm{C}$ NMR $\left(125 \mathrm{MHz}, \mathrm{MeOD}-d_{4}\right) \delta 4.3,116.9,119.2$, 120.1, 122.9, 129.6, 163.2, 163.5. LRMS $\left(\mathrm{ES}^{+}\right) \mathrm{m} / z 250.1[\mathrm{M}+\mathrm{H}]^{+}$. HRMS calcd for $\mathrm{C}_{14} \mathrm{H}_{12} \mathrm{~N}_{5}[\mathrm{M}+\mathrm{H}]^{+} 250.1087$, found 250.1085 .

$\mathrm{N}$-Phenyl-6-(phenylethynyl)-9H-purin-2-amine (49). Prepared in accordance with general procedure E from $47(0.055 \mathrm{~g}, 0.14 \mathrm{mmol})$. Recrystallization from EtOAc-petrol gave 49 as a yellow solid $(22 \mathrm{mg}$, $51 \%)$; mp $206-208{ }^{\circ} \mathrm{C}$ (dec); UV $\lambda_{\max }(\mathrm{EtOH}) 281 \mathrm{~nm}$. IR $\left(\mathrm{cm}^{-1}\right)$ $1532,1574,2208,3226 .{ }^{1} \mathrm{H}$ NMR $\left(500 \mathrm{MHz}, \mathrm{MeOD}-d_{4}\right) \delta 6.79(1 \mathrm{H}$, $\left.\mathrm{t}, J=7.3 \mathrm{~Hz}, \mathrm{H}-4^{\prime}\right), 7.11\left(2 \mathrm{H}, \mathrm{dd}, J=7.8,8.1 \mathrm{~Hz}, \mathrm{H}-2^{\prime}\right.$ and $\left.\mathrm{H}-6^{\prime}\right)$, 7.23-7.32 (3H, m, H-3', H-5' and Ar-H), 7.54-7.58 (4H, m, $4 \times$ $\mathrm{ArH}), 8.02(1 \mathrm{H}, \mathrm{s}, \mathrm{H}-8) .{ }^{13} \mathrm{C}$ NMR $\left(125 \mathrm{MHz}, \mathrm{MeOD}-d_{4}\right) \delta 97.3$, 120.2, 122.8, 122.9, 129.6, 129.8, 131.2, 133.5, 141.8, 158.6. LRMS $\left(\mathrm{ES}^{+}\right) \mathrm{m} / z$ 312.2 $[\mathrm{M}+\mathrm{H}]^{+}$. HRMS calculated for $\mathrm{C}_{19} \mathrm{H}_{14} \mathrm{~N}_{5}[\mathrm{M}+\mathrm{H}]^{+}$ 312.1244, found 312.1242.

6-Ethyl-2-fluoro-9-(tetrahydro-2H-pyran-2-yl)-9H-purine (50). To a solution of $43(0.05 \mathrm{~g}, 0.20 \mathrm{mmol})$ and quinoline $(20 \mu \mathrm{L})$ in EtOAc $(5 \mathrm{~mL})$ was added Lindlar's catalyst $(10 \mathrm{mg}, 20 \% \mathrm{w} / \mathrm{w})$, and the mixture was stirred for $2 \mathrm{~h}$ under an atmosphere of $\mathrm{H}_{2}$. The suspension was filtered through Celite, eluting with methanol (30 $\mathrm{mL}$ ), and volatiles were removed in vacuo. Purification by chromatography (silica; 50\% EtOAc:petrol) yielded $\mathbf{5 0}$ as a colorless oil (50 mg, 99\%); UV $\lambda_{\max }(\mathrm{EtOH}) 264 \mathrm{~nm}$. IR $\left(\mathrm{cm}^{-1}\right) 2946,2860$, 2364, 2338, 1604. ${ }^{1} \mathrm{H}$ NMR (500 MHz, DMSO- $\left.d_{6}\right) \delta 1.35(3 \mathrm{H}, \mathrm{t}, J=$ $\left.7.5 \mathrm{~Hz}, \mathrm{CH}_{3} \mathrm{CH}_{2}\right), 1.59\left(2 \mathrm{H}, \mathrm{m}, \mathrm{CH}_{2}\right), 1.97(1 \mathrm{H}, \mathrm{m}, \mathrm{CH}), 1.99(2 \mathrm{H}, \mathrm{d}$, $\left.J=10.5 \mathrm{~Hz}, \mathrm{CH}_{2}\right), 2.50(1 \mathrm{H}, \mathrm{m}, \mathrm{CH}), 3.01(2 \mathrm{H}, \mathrm{q}, J=7.5 \mathrm{~Hz}$, $\left.\mathrm{CH}_{3} \mathrm{CH}_{2}\right), 3.73(1 \mathrm{H}, \mathrm{m}, \mathrm{CH}), 4.04(1 \mathrm{H}, \mathrm{d}, J=10.5 \mathrm{~Hz}, \mathrm{CH}), 5.69$ $(1 \mathrm{H}, \mathrm{d}, J=10.5 \mathrm{~Hz}, \mathrm{CH}), 8.74(1 \mathrm{H}, \mathrm{s}, \mathrm{H}-8) .{ }^{13} \mathrm{C}$ NMR $(500 \mathrm{MHz}$,
DMSO- $\left.d_{6}\right) \delta 12.3,22.7,24.8,26.5,31.8,68.9,82.1,142.1,157.9$, 159.6, 167.4, 167.5. LRMS $\left(\mathrm{ES}^{+}\right) \mathrm{m} / z 251.0[\mathrm{M}+\mathrm{H}]^{+}$.

6-Ethyl-2-fluoro-9H-purine (51). Prepared in accordance with general procedure E from $50(0.18 \mathrm{~g}, 0.72 \mathrm{mmol})$. Purification by chromatography (silica; $25 \%$ EtOAc:petrol) yielded $\mathbf{5 1}$ as a white solid $(0.117 \mathrm{~g}, 98 \%) ; \mathrm{mp} 146-148^{\circ} \mathrm{C}$; UV $\lambda_{\max }(\mathrm{EtOH}) 269 \mathrm{~nm}$. IR $\left(\mathrm{cm}^{-1}\right)$ $1676,1616,1573 .{ }^{1} \mathrm{H}$ NMR $\left(400 \mathrm{MHz}, \mathrm{DMSO}-d_{6}\right) \delta 1.30(3 \mathrm{H}, \mathrm{t}, J=$ $\left.7.5 \mathrm{~Hz}, \mathrm{CH}_{3} \mathrm{CH}_{2}\right), 3.00\left(2 \mathrm{H}, \mathrm{q}, J=7.5 \mathrm{~Hz}, \mathrm{CH}_{3} \mathrm{CH}_{2}\right), 8.17(1 \mathrm{H}, \mathrm{s}, \mathrm{H}-$ 8). ${ }^{13} \mathrm{C}$ NMR (125 MHz, DMSO- $\left.d_{6}\right) \delta 11.7,25.9,68.3,116.0,118.4$, $146.4,158.0\left(\mathrm{~d}, J_{\mathrm{C}-\mathrm{F}}=206.0 \mathrm{~Hz}\right), 158.3\left(\mathrm{~d}, J_{\mathrm{C}-\mathrm{F}}=31.5 \mathrm{~Hz}\right)$. LRMS $\left(\mathrm{ES}^{+}\right) \mathrm{m} / z 167.7[\mathrm{M}+\mathrm{H}]^{+}$.

4-(6-Ethyl-9H-purin-2-ylamino)benzenesulfonamide (52). Prepared by general procedure A from $\mathbf{5 1}(0.081 \mathrm{~g}, 0.49 \mathrm{mmol})$, following purification by chromatography (silica; $50 \%$ EtOAc:petrol), as a white solid ( $33 \mathrm{mg}, 21 \%) ; \mathrm{mp} 291-293^{\circ} \mathrm{C}$; UV $\lambda_{\max }(\mathrm{EtOH}) 318$, 287, $212 \mathrm{~nm}$. IR $\left(\mathrm{cm}^{-1}\right) 3377,3060,2852,1388,1158 .{ }^{1} \mathrm{H}$ NMR (500 $\left.\mathrm{MHz}, \mathrm{DMSO}-d_{6}\right) \delta 1.35\left(3 \mathrm{H}, \mathrm{t}, J=6.0 \mathrm{~Hz}, \mathrm{CH}_{3} \mathrm{CH}_{2}\right), 3.00(2 \mathrm{H}, \mathrm{q}, J=$ $\left.6.0 \mathrm{~Hz}, \mathrm{CH}_{3} \mathrm{CH}_{2}\right), 7.14\left(2 \mathrm{H}\right.$, br s, $\left.\mathrm{SO}_{2} \mathrm{NH}_{2}\right), 7.69(2 \mathrm{H}, \mathrm{d}, J=7.5 \mathrm{~Hz}$, H-2 ${ }^{\prime}$ and $\left.\mathrm{H}-6^{\prime}\right), 7.99\left(2 \mathrm{H}, \mathrm{d}, J=7.5 \mathrm{~Hz}, \mathrm{H}-3^{\prime}\right.$ and $\left.\mathrm{H}-5^{\prime}\right), 8.17(1 \mathrm{H}, \mathrm{s}$, $\mathrm{H}-8), 9.89(1 \mathrm{H}, \mathrm{br} \mathrm{s}, \mathrm{NH}) .{ }^{13} \mathrm{C}$ NMR (125 MHz, DMSO- $\left.d_{6}\right) \delta 12.3$, 26.0, 117.0, 118.9, 125.4, 126.4, 135.2, 141.2, 144.4, 154.0, 155.5 . LRMS $\left(\mathrm{ES}^{+}\right) \mathrm{m} / z 318.6[\mathrm{M}+\mathrm{H}]^{+}$. HRMS calcd for $\mathrm{C}_{13} \mathrm{H}_{14} \mathrm{~N}_{6} \mathrm{O}_{2} \mathrm{~S}[\mathrm{M}$ $+\mathrm{H}]^{+}$319.097076, found 319.09788.

6-Cyclopropyl-2-fluoro-9-(tetrahydro-2H-pyran-2-yl)-9H-purine (53). The title compound was prepared from $36(0.04 \mathrm{~g}, 0.27 \mathrm{mmol})$ and potassium cyclopropyltrifluoroborate $(50 \mathrm{mg}, 0.19 \mathrm{mmol})$ following general procedure G. Purification by chromatography (silica; 50\% EtOAc:petrol) afforded $\mathbf{5 3}$ as a colorless oil (21 mg, 42\%); UV $\lambda_{\max }(\mathrm{EtOH}) 211,325,391 \mathrm{~nm}$. IR $\left(\mathrm{cm}^{-1}\right) 2986 .{ }^{1} \mathrm{H}$ NMR $(400 \mathrm{MHz}$, DMSO-d $\left.d_{6}\right) \delta 1.25\left(4 \mathrm{H}, \mathrm{d}, J=6.5 \mathrm{~Hz}\right.$, cyclopropyl $\left.2 \times \mathrm{CH}_{2}\right), 1.55(2 \mathrm{H}$, $\left.\mathrm{m}, \mathrm{CH}_{2}\right), 1.72(1 \mathrm{H}, \mathrm{m}, \mathrm{CH}), 1.95\left(2 \mathrm{H}, \mathrm{d}, J=10.5 \mathrm{~Hz}, \mathrm{CH}_{2}\right), 2.27(1 \mathrm{H}$, m, CH), $2.67(1 \mathrm{H}, \mathrm{m}$, cyclopropyl $\mathrm{CH}), 3.70(1 \mathrm{H}, \mathrm{m}, \mathrm{CH}), 3.99(1 \mathrm{H}$, d, $J=10.5 \mathrm{~Hz}, \mathrm{CH}), 5.63(1 \mathrm{H}, \mathrm{d}, J=10.5 \mathrm{~Hz}, \mathrm{CH}), 8.70(1 \mathrm{H}, \mathrm{s}, \mathrm{H}-8)$. ${ }^{13} \mathrm{C}$ NMR (500 MHz, DMSO- $\left.d_{6}\right) \delta 11.9,13.2,22.2,24.4,29.7,67.7$, 81.3, 130.9, 144.1, 151.8, 157.6, 159.8, 176.9. LRMS $\left(\mathrm{ES}^{+}\right) \mathrm{m} / z 262.4$ $[\mathrm{M}+\mathrm{H}]^{+}$.

6-Cyclopropyl-2-fluoro-9H-purine (54). The title compound was prepared from $53(0.19 \mathrm{~g}, 0.72 \mathrm{mmol})$ employing general procedure $\mathrm{E}$. Purification by chromatography (silica; EtOAc) afforded 54 as a white solid (0.12 g, 95\%); mp 137-139 ${ }^{\circ} \mathrm{C}$; UV $\lambda_{\max }(\mathrm{EtOH}) 323,213 \mathrm{~nm}$. IR $\left(\mathrm{cm}^{-1}\right)$ 3062. ${ }^{1} \mathrm{H}$ NMR $\left(400 \mathrm{MHz}\right.$, DMSO- $\left.d_{6}\right) \delta 1.11(4 \mathrm{H}, \mathrm{d}, J=$ $6.5 \mathrm{~Hz}$, cyclopropyl $\left.2 \times \mathrm{CH}_{2}\right), 2.51(1 \mathrm{H}, \mathrm{m}$, cyclopropyl $\mathrm{CH}), 8.40$ $(1 \mathrm{H}, \mathrm{s}, \mathrm{H}-8) .{ }^{13} \mathrm{C}$ NMR $\left(400 \mathrm{MHz}, \mathrm{DMSO}-d_{6}\right) \delta 11.6,13.1,131.2$, 144.2, 151.8, 157.6, 159.8. LRMS $\left(\mathrm{ES}^{+}\right) \mathrm{m} / z 179.0[\mathrm{M}+\mathrm{H}]^{+}$.

4-(6-Cyclopropyl-9H-purin-2-ylamino)benzenesulfonamide (55). Synthesized following general procedure A from 54 (0.086 g, 0.49 $\mathrm{mmol}$ ). Purification by chromatography (silica; EtOAc) afforded 55 as a pale-yellow solid (24 mg, 15\%); mp 291-293 ${ }^{\circ} \mathrm{C}$; UV $\lambda_{\max }(\mathrm{EtOH})$ 315.5, 289, $213 \mathrm{~nm}$. IR $\left(\mathrm{cm}^{-1}\right)$ 3389, 3062, 2921, 2851, 1727, 1604, $1579,1531,1379,1149 .{ }^{1} \mathrm{H}$ NMR $\left(500 \mathrm{MHz}, \mathrm{DMSO}-d_{6}\right) \delta 1.23(4 \mathrm{H}$, $\mathrm{d}, J=6.5 \mathrm{~Hz}$, cyclopropyl $\left.2 \times \mathrm{CH}_{2}\right), 1.35(1 \mathrm{H}, \mathrm{m}$, cyclopropyl $\mathrm{CH})$, $7.18\left(2 \mathrm{H}, \mathrm{br} \mathrm{s}, \mathrm{SO}_{2} \mathrm{NH}_{2}\right), 7.74\left(2 \mathrm{H}, \mathrm{d}, J=8.0 \mathrm{~Hz}, \mathrm{H}-2^{\prime}\right.$ and $\left.\mathrm{H}-6^{\prime}\right), 7.99$ $\left(2 \mathrm{H}, \mathrm{d}, J=8.0 \mathrm{~Hz}, \mathrm{H}-3^{\prime}\right.$ and $\left.\mathrm{H}-5^{\prime}\right), 8.22(1 \mathrm{H}, \mathrm{s}, \mathrm{H}-8), 9.75(1 \mathrm{H}, \mathrm{br} \mathrm{s}$, $\mathrm{NH}) .{ }^{13} \mathrm{C}$ NMR (125 MHz, DMSO-d $d_{6} \delta 10.5,13.0,117.1,126.4$, 126.6, 141.1, 144.4, 151.8, 155.5, 162.9. LRMS $\left(\mathrm{ES}^{+}\right) \mathrm{m} / z$ 331.1 [M + $\mathrm{H}]^{+}$. HRMS calcd for $\mathrm{C}_{14} \mathrm{H}_{15} \mathrm{~N}_{6} \mathrm{O}_{2} \mathrm{~S}[\mathrm{M}+\mathrm{H}]^{+} 331.0977$, found 331.0980 .

2-Fluoro-6-phenyl-9-(tetrahydro-2H-pyran-2-yl)-9H-purine (56). Synthesized in accordance with general procedure G from $36(0.20$ g, $0.78 \mathrm{mmol}$ ) and phenylboronic acid (0.133 g, $1.09 \mathrm{mmol})$. Purification by chromatography (silica; $20 \%$ EtOAc:petrol) gave 56 as a white solid $(0.30 \mathrm{~g}, 95 \%) ; \mathrm{mp} 138-140{ }^{\circ} \mathrm{C}$; UV $\lambda_{\max }(\mathrm{EtOH}) 306$, 269, $203 \mathrm{~nm}$. IR $\left(\mathrm{cm}^{-1}\right)$ 3117, 2956, 2858, 1586. ${ }^{1} \mathrm{H}$ NMR (300 MHz, DMSO- $\left.d_{6}\right) \delta 1.60\left(2 \mathrm{H}, \mathrm{m}, \mathrm{CH}_{2}\right), 1.76(1 \mathrm{H}, \mathrm{m}, \mathrm{CH}), 2.02(2 \mathrm{H}, \mathrm{m}$, $\left.\mathrm{CH}_{2}\right), 2.29(1 \mathrm{H}, \mathrm{s}, \mathrm{CH}), 3.77(1 \mathrm{H}, \mathrm{t}, J=12.0 \mathrm{~Hz}, \mathrm{CH}), 4.04(1 \mathrm{H}, \mathrm{d}, J$ $=12.0 \mathrm{~Hz}, \mathrm{CH}), 5.74(1 \mathrm{H}, \mathrm{d}, J=12.0 \mathrm{~Hz}, \mathrm{CH}), 7.64,\left(3 \mathrm{H}, \mathrm{m}, \mathrm{H}-3^{\prime \prime}\right.$, H-4" and H-5"), 8.79 (2H, dd, $J=3.0,7.5 \mathrm{~Hz}, \mathrm{H}-2^{\prime \prime}$ and H-6" $), 8.25$ $(1 \mathrm{H}, \mathrm{s}, \mathrm{H}-8) .{ }^{13} \mathrm{C}$ NMR $\left(125 \mathrm{MHz}\right.$, DMSO- $\left.d_{6}\right) \delta 22.2,24.4,29.7,67.7$, $81.3,128.9,129.3,129.5,132.0,134.0,145.4,154.3\left(\mathrm{~d}, J_{\mathrm{C}-\mathrm{F}}=17.5\right.$ 
$\mathrm{Hz}), 155.5\left(\mathrm{~d}, J_{\mathrm{C}-\mathrm{F}}=144.0 \mathrm{~Hz}\right), 157.0$, 158.7. LRMS $\left(\mathrm{ES}^{+}\right) \mathrm{m} / z 299.3$ $[\mathrm{M}+\mathrm{H}]^{+}$

2-Fluoro-6-(3-methoxyphenyl)-9-(tetrahydro-2H-pyran-2-yl)-9Hpurine (57). Prepared according to general procedure $\mathrm{G}$ from $36(0.20$ $\mathrm{g}, 0.78 \mathrm{mmol})$ and 4-methoxyphenylboronic acid $(0.16 \mathrm{~g}, 1.09 \mathrm{mmol})$. Purification by chromatography (silica; $30 \%$ EtOAc:petrol) gave $\mathbf{5 7}$ as a white solid (0.29 g, 80\%); mp $133-135^{\circ} \mathrm{C}$; UV $\lambda_{\max }(\mathrm{EtOH}) 306$, $271,204 \mathrm{~nm}$. IR $\left(\mathrm{cm}^{-1}\right) 3089,2938,2857,1761,1586,1516 .{ }^{1} \mathrm{H}$ NMR $\left(300 \mathrm{MHz}, \mathrm{DMSO}-d_{6}\right) \delta 1.60\left(2 \mathrm{H}, \mathrm{m}, \mathrm{CH}_{2}\right), 1.76(1 \mathrm{H}, \mathrm{m}, \mathrm{CH}), 2.02$ $\left(2 \mathrm{H}, \mathrm{m}, \mathrm{CH}_{2}\right), 2.29(1 \mathrm{H}, \mathrm{s}, \mathrm{CH}), 3.77(1 \mathrm{H}, \mathrm{t}, J=12.0 \mathrm{~Hz}, \mathrm{CH}), 3.89$ $\left(3 \mathrm{H}, \mathrm{s}, \mathrm{OCH}_{3}\right), 4.04(1 \mathrm{H}, \mathrm{d}, J=12.0 \mathrm{~Hz}, \mathrm{CH}), 5.74(1 \mathrm{H}, \mathrm{d}, J=12.0$ $\mathrm{Hz}, \mathrm{CH}), 7.18\left(1 \mathrm{H}, \mathrm{m}, \mathrm{H}-2^{\prime \prime}\right), 7.50-7.55\left(1 \mathrm{H}, \mathrm{m}, \mathrm{H}-5^{\prime \prime}\right), 8.45-8.49$ $\left(2 \mathrm{H}, \mathrm{m}, \mathrm{H}-4^{\prime \prime}, \mathrm{H}-6^{\prime \prime}\right), 8.78$ (1H, s, H-8). ${ }^{13} \mathrm{C}$ NMR (125 MHz, DMSO-d $d_{6} \delta 22.2,24.4,29.7,55.3,67.7,81.3,114.4,117.9,122.0$, $129.4,130.0,135.3,145.4,154.3\left(\mathrm{~d}, J_{\mathrm{C}-\mathrm{F}}=17.5 \mathrm{~Hz}\right), 155.0\left(\mathrm{~d}, J_{\mathrm{C}-\mathrm{F}}=\right.$ $160.0 \mathrm{~Hz}), 156.9,158.6,159.4$. LRMS $\left(\mathrm{ES}^{+}\right) \mathrm{m} / z 334.2[\mathrm{M}+\mathrm{H}]^{+}$.

2-Fluoro-6-(4-methoxyphenyl)-9-(tetrahydro-2H-pyran-2-yl)-9Hpurine (58). Synthesized in accordance with general procedure $\mathrm{G}$ from $36(0.20 \mathrm{~g}, 0.78 \mathrm{mmol})$ and 4-methoxyphenylboronic acid $(0.16 \mathrm{~g}$, $1.09 \mathrm{mmol}$ ). Purification by chromatography (silica; 30\% EtOAc:petrol) afforded 58 as a white solid $(0.35 \mathrm{~g}, 98 \%)$; mp $131-133{ }^{\circ} \mathrm{C}$; UV $\lambda_{\max }(\mathrm{EtOH}) 306,271,204 \mathrm{~nm}$. IR $\left(\mathrm{cm}^{-1}\right)$ 3089, 2938, 2857, 1761, 1586, 1516. ${ }^{1} \mathrm{H}$ NMR (300 MHz, DMSO- $\left.d_{6}\right) \delta 1.60\left(2 \mathrm{H}, \mathrm{m}, \mathrm{CH}_{2}\right)$, $1.76(1 \mathrm{H}, \mathrm{m}, \mathrm{CH}), 2.02\left(2 \mathrm{H}, \mathrm{m}, \mathrm{CH}_{2}\right), 2.29(1 \mathrm{H}, \mathrm{s}, \mathrm{CH}), 3.77(1 \mathrm{H}, \mathrm{t}, J$ $=12.0 \mathrm{~Hz}, \mathrm{CH}), 3.88\left(3 \mathrm{H}, \mathrm{s}, \mathrm{OCH}_{3}\right), 4.04(1 \mathrm{H}, \mathrm{d}, J=12.0 \mathrm{~Hz}, \mathrm{CH})$, $5.74(1 \mathrm{H}, \mathrm{d}, J=12.0 \mathrm{~Hz}, \mathrm{CH}), 7.15-7.18\left(2 \mathrm{H}, \mathrm{d}, J=9.0 \mathrm{~Hz}, \mathrm{H}-3^{\prime \prime}\right.$ and $\left.\mathrm{H}-5^{\prime \prime}\right), 8.79-8.81\left(2 \mathrm{H}, \mathrm{d}, J=9.0 \mathrm{~Hz}, \mathrm{H}-2^{\prime \prime}\right.$ and $\left.\mathrm{H}-6^{\prime \prime}\right), 8.85(1 \mathrm{H}, \mathrm{s}, \mathrm{H}-$ 8). ${ }^{13} \mathrm{C}$ NMR (125 MHz, DMSO- $\left.d_{6}\right) \delta 22.2,24.4,29.7,55.5,67.7$, 81.2, 114.3, 126.5, 128.5, 131.5, 144.7, $153.9\left(\mathrm{~d}, J_{\mathrm{C}-\mathrm{F}}=17.5 \mathrm{~Hz}\right), 155.2$ $\left(\mathrm{d}, J_{\mathrm{C}-\mathrm{F}}=160.0 \mathrm{~Hz}\right), 157.0,158.7,162.4$. LRMS $\left(\mathrm{ES}^{+}\right) \mathrm{m} / z$ 329.2 [M $+\mathrm{H}]^{+}$.

6-(3-Biphenyl)-2-fluoro-9-(tetrahydro-2H-pyran-2-yl)-9H-purine (59). Prepared following general procedure G from $36(0.20 \mathrm{~g}, 0.78$ $\mathrm{mmol})$ and 3-biphenylboronic acid $(0.22 \mathrm{~g}, 1.09 \mathrm{mmol})$. Purification by chromatography (silica; $20 \%$ EtOAc:petrol) furnished $\mathbf{5 9}$ as a paleyellow solid (0.33 g, 80\%); mp 221-223 ${ }^{\circ} \mathrm{C}$; UV $\lambda_{\max }(\mathrm{EtOH}) 346$, $274 \mathrm{~nm}$. IR $\left(\mathrm{cm}^{-1}\right)$ 2992, 2844, 2601, 1589. ${ }^{1} \mathrm{H}$ NMR $(300 \mathrm{MHz}$, DMSO-d 6 ) $\delta 1.60\left(2 \mathrm{H}, \mathrm{m}, \mathrm{CH}_{2}\right), 1.76(1 \mathrm{H}, \mathrm{m}, \mathrm{CH}), 2.02(2 \mathrm{H}, \mathrm{m}$, $\left.\mathrm{CH}_{2}\right), 2.29(1 \mathrm{H}, \mathrm{s}, \mathrm{CH}), 3.77(1 \mathrm{H}, \mathrm{t}, J=12.0 \mathrm{~Hz}, \mathrm{CH}), 4.04(1 \mathrm{H}, \mathrm{d}, J$ $=12.0 \mathrm{~Hz}, \mathrm{CH}), 5.79(1 \mathrm{H}, \mathrm{d}, J=12.0 \mathrm{~Hz}, \mathrm{CH}), 7.45(1 \mathrm{H}, \mathrm{d}, J=7.0$ $\left.\mathrm{Hz}, \mathrm{H}-6^{\prime \prime}\right), 7.56\left(1 \mathrm{H}, \mathrm{dd}, J=7.0,7.1 \mathrm{~Hz}, \mathrm{H}-5^{\prime \prime}\right), 7.78(5 \mathrm{H}, \mathrm{m}, \mathrm{Ph}), 8.40$ $\left(1 \mathrm{H}, \mathrm{s}, \mathrm{H}-2^{\prime \prime}\right), 8.84\left(1 \mathrm{H}, \mathrm{d}, J=7.1 \mathrm{~Hz}, \mathrm{H}-4^{\prime \prime}\right), 9.18(1 \mathrm{H}, \mathrm{s}, \mathrm{H}-8) .{ }^{13} \mathrm{C}$ NMR (125 MHz, DMSO-d $\left.d_{6}\right) \delta 22.2,24.4,29.7,67.7,81.3,126.8$, $127.8,127.9,128.4,129.1,129.5,129.6,130.3,134.7,139.6,140.8$, $145.6,154.4\left(\mathrm{~d}, J_{\mathrm{C}-\mathrm{F}}=17.5 \mathrm{~Hz}\right), 155.2\left(\mathrm{~d}, J_{\mathrm{C}-\mathrm{F}}=116.0 \mathrm{~Hz}\right), 157.0$, 158.5. LRMS $\left(\mathrm{ES}^{+}\right) \mathrm{m} / z$ 375.1 $[\mathrm{M}+\mathrm{H}]^{+}$.

2-Fluoro-6-(3-piperonyl)-9-(tetrahydro-2H-pyran-2-yl)-9H-purine (60). Synthesized in accordance with general procedure $\mathrm{G}$ from 36 $(0.20 \mathrm{~g}, 0.78 \mathrm{mmol})$ and 3,4-(methylenedioxy)phenylboronic acid (0.18 g, $1.09 \mathrm{mmol})$. Purification by chromatography (silica; $30 \%$ EtOAc:petrol) yielded 60 as a white solid $(0.21 \mathrm{~g}, 56 \%)$; mp 151-153 ${ }^{\circ} \mathrm{C}$; UV $\lambda_{\max }(\mathrm{EtOH}) 351,284 \mathrm{~nm}$. IR $\left(\mathrm{cm}^{-1}\right) 3011,2917,1721,1616$, 1511. ${ }^{1} \mathrm{H}$ NMR $\left(300 \mathrm{MHz}, \mathrm{DMSO}-d_{6}\right) \delta 1.60\left(2 \mathrm{H}, \mathrm{m}, \mathrm{CH}_{2}\right), 1.76$ $(1 \mathrm{H}, \mathrm{m}, \mathrm{CH}), 2.02\left(2 \mathrm{H}, \mathrm{m}, \mathrm{CH}_{2}\right), 2.29(1 \mathrm{H}, \mathrm{s}, \mathrm{CH}), 3.73(1 \mathrm{H}, \mathrm{t}, J=$ $12.0 \mathrm{~Hz}, \mathrm{CH}), 4.04(1 \mathrm{H}, \mathrm{d}, J=12.0 \mathrm{~Hz}, \mathrm{CH}), 5.73(1 \mathrm{H}, \mathrm{d}, J=12.0 \mathrm{~Hz}$, $\mathrm{CH}), 6.17\left(2 \mathrm{H}, \mathrm{s}, \mathrm{OCH}_{2} \mathrm{O}\right), 7.14-7.17\left(1 \mathrm{H}, \mathrm{d}, J=8.0 \mathrm{~Hz}, \mathrm{H}-5^{\prime \prime}\right), 8.28$ $\left(1 \mathrm{H}, \mathrm{s}, \mathrm{H}-2^{\prime \prime}\right), 8.48-8.51\left(1 \mathrm{H}, \mathrm{d}, J=8.0 \mathrm{~Hz}, \mathrm{H}-6^{\prime \prime}\right), 8.82(1 \mathrm{H}, \mathrm{s}, \mathrm{H}-8)$. ${ }^{13} \mathrm{C}$ NMR (125 MHz, DMSO-d $\left.d_{6}\right) \delta 22.2,24.4,29.7,67.7,81.2,101.9$, 108.6, 125.4, 128.0, 128.6, 144.9, 147.8, 150.6, 154.1, 154.8, 156.9, 158.7. LRMS $\left(\mathrm{ES}^{+}\right) \mathrm{m} / z 343.1[\mathrm{M}+\mathrm{H}]^{+}$.

6-(4-Dibenzofuryl)-2-fluoro-9-(tetrahydro-2H-pyran-2-yl)-9H-purine (61). Prepared according to general procedure $\mathrm{G}$ from $36(0.20 \mathrm{~g}$, $0.78 \mathrm{mmol})$ and 4-dibenzofuranboronic acid (0.23 g, $1.09 \mathrm{mmol})$. Purification by chromatography (silica; $30 \%$ EtOAc:petrol) yielded $\mathbf{6 1}$ as a cream solid $(0.39 \mathrm{~g}, 92 \%) ; \mathrm{mp} 168-170{ }^{\circ} \mathrm{C}$; $\mathrm{UV} \lambda_{\max }(\mathrm{EtOH})$ 398, 256, $202 \mathrm{~nm}$. IR $\left(\mathrm{cm}^{-1}\right) 3092,2589,1579 .{ }^{1} \mathrm{H}$ NMR $(300 \mathrm{MHz}$, DMSO-d $\left.d_{6}\right) \delta 1.60\left(2 \mathrm{H}, \mathrm{m}, \mathrm{CH}_{2}\right), 1.76(1 \mathrm{H}, \mathrm{m}, \mathrm{CH}), 2.02(2 \mathrm{H}, \mathrm{m}$, $\left.\mathrm{CH}_{2}\right), 2.29(1 \mathrm{H}, \mathrm{s}, \mathrm{CH}), 3.77(1 \mathrm{H}, \mathrm{t}, J=12 \mathrm{~Hz}, \mathrm{CH}), 4.04(1 \mathrm{H}, \mathrm{d}, J=$ $12.0 \mathrm{~Hz}, \mathrm{CH}), 5.79(1 \mathrm{H}, \mathrm{d}, J=12.0 \mathrm{~Hz}, \mathrm{CH}), 7.45-7.47(1 \mathrm{H}, \mathrm{dd}, J=$ 7.0, $\left.7.1 \mathrm{~Hz}, \mathrm{H}-2^{\prime \prime}\right), 7.52-7.62\left(1 \mathrm{H}, \mathrm{dd}, J=8.0,8.1 \mathrm{~Hz}, \mathrm{H}-7^{\prime \prime}\right), 7.61-$ $7.71\left(1 \mathrm{H}, \mathrm{dd}, J=8.0,8.1 \mathrm{~Hz}, \mathrm{H}-8^{\prime \prime}\right), 7.76-7.79(1 \mathrm{H}, \mathrm{d}, J=8.0 \mathrm{~Hz}, \mathrm{H}-$ $\left.9^{\prime \prime}\right), 8.25-8.27\left(1 \mathrm{H}, \mathrm{d}, J=7.1 \mathrm{~Hz}, \mathrm{H}-3^{\prime \prime}\right), 8.42-8.44(1 \mathrm{H}, \mathrm{d}, J=8.0$ $\left.\mathrm{Hz}, \mathrm{H}-6^{\prime \prime}\right), 8.55-8.58\left(1 \mathrm{H}, \mathrm{d}, J=7.5 \mathrm{~Hz}, \mathrm{H}-1^{\prime \prime}\right), 8.93(1 \mathrm{H}, \mathrm{s}, \mathrm{H}-8) .{ }^{13} \mathrm{C}$ NMR $\left(125 \mathrm{MHz}\right.$, DMSO- $\left.d_{6}\right) \delta 22.2,24.5,29.7,67.8,68.3,81.4,111.9$, $119.3,121.3,122.9,123.1,123.4,124.3,125.2,128.1,130.1,145.6$, $153.1,154.7\left(\mathrm{~d}, J_{\mathrm{C}-\mathrm{F}}=18.0 \mathrm{~Hz}\right), 155.7\left(\mathrm{~d}, J_{\mathrm{C}-\mathrm{F}}=160.0 \mathrm{~Hz}\right), 156.9$, 158.5. LRMS $\left(\mathrm{ES}^{+}\right) \mathrm{m} / z$ 389.4 $[\mathrm{M}+\mathrm{H}]^{+}$.

2-Fluoro-6-(1-thianthrenyl)-9-(tetrahydro-2H-pyran-2-yl)-9H-purine (62). Prepared in accordance with general procedure $\mathrm{G}$ from 36 $(0.20 \mathrm{~g}, 0.78 \mathrm{mmol})$ and 1-thianthreneboronic acid (0.28 g, 1.09 mmol). Purification by chromatography (silica; 30\% EtOAc:petrol) afforded 62 as an orange solid $(0.43 \mathrm{~g}, 90 \%)$; mp $164-166{ }^{\circ} \mathrm{C}$; UV $\lambda_{\max }(\mathrm{EtOH}) 396,224 \mathrm{~nm} . \mathrm{IR}\left(\mathrm{cm}^{-1}\right)$ 3092, 2814, 2589, $1611,1497$. ${ }^{1} \mathrm{H}$ NMR $\left(300 \mathrm{MHz}, \mathrm{DMSO}-d_{6}\right) \delta 1.60\left(2 \mathrm{H}, \mathrm{m}, \mathrm{CH}_{2}\right), 1.76(1 \mathrm{H}, \mathrm{m}$, $\mathrm{CH}), 2.02\left(2 \mathrm{H}, \mathrm{m}, \mathrm{CH}_{2}\right), 2.29(1 \mathrm{H}, \mathrm{s}, \mathrm{CH}), 3.77(1 \mathrm{H}, \mathrm{t}, J=12.0 \mathrm{~Hz}$, $\mathrm{CH}), 4.04(1 \mathrm{H}, \mathrm{d}, J=12.0 \mathrm{~Hz}, \mathrm{CH}), 5.79(1 \mathrm{H}, \mathrm{d}, J=12.0 \mathrm{~Hz}, \mathrm{CH})$, $7.21-7.30\left(1 \mathrm{H}, \mathrm{dd}, J=7.4,7.5 \mathrm{~Hz}, \mathrm{H}-3^{\prime \prime}\right), 7.31-7.39(1 \mathrm{H}, \mathrm{dd}, J=7.5$, $\left.7.6 \mathrm{~Hz}, \mathrm{H}-7^{\prime \prime}\right), 7.37-7.45\left(1 \mathrm{H}, \mathrm{d}, J=7.5 \mathrm{~Hz}, \mathrm{H}-9^{\prime \prime}\right), 7.51-7.58(1 \mathrm{H}$, dd, $\left.J=7.5,7.6 \mathrm{~Hz}, \mathrm{H}-8^{\prime \prime}\right), 7.59-7.65\left(1 \mathrm{H}, \mathrm{d}, J=7.5 \mathrm{~Hz}, \mathrm{H}-6^{\prime \prime}\right), 7.79$ $\left(1 \mathrm{H}, \mathrm{d}, J=7.4 \mathrm{~Hz}, \mathrm{H}-2^{\prime \prime}\right), 7.89\left(1 \mathrm{H}, \mathrm{d}, J=7.5 \mathrm{~Hz}, \mathrm{H}-4^{\prime \prime}\right), 8.93(1 \mathrm{H}, \mathrm{s}$, H-8). ${ }^{13} \mathrm{C}$ NMR (125 MHz, DMSO-d $\left.d_{6}\right) \delta 22.2,24.4,29.5,67.7,68.3$, $81.5127 .5,128.2,128.5,128.7,128.9,130.6,130.9,131.4,134.5,134.7$, $135.1,135.5,136.4,146.2,153.8\left(\mathrm{~d}, J_{\mathrm{C}-\mathrm{F}}=17.5 \mathrm{~Hz}\right), 156.2,155.0(\mathrm{~d}$, $\left.J_{\mathrm{C}-\mathrm{F}}=150.0 \mathrm{~Hz}\right)$, 157.2. LRMS $\left(\mathrm{ES}^{+}\right) \mathrm{m} / z$ 437.2 $[\mathrm{M}+\mathrm{H}]^{+}$.

2-Fluoro-6-phenyl-9H-purine (63). Prepared from 56 (0.22 g, 0.72 $\mathrm{mmol}$ ) following general procedure E. Repeated trituration of the precipitated solid with water gave $63(0.14 \mathrm{~g}, 90 \%)$; mp $273-275^{\circ} \mathrm{C}$; UV $\lambda_{\max }(\mathrm{EtOH}) 349 \mathrm{~nm}$. IR $\left(\mathrm{cm}^{-1}\right) 2820,1599,1560 .{ }^{1} \mathrm{H}$ NMR $(300$ MHz, DMSO- $\left.d_{6}\right) \delta 7.62\left(3 \mathrm{H}, \mathrm{m}, \mathrm{H}-3^{\prime \prime}, \mathrm{H}-4^{\prime \prime}\right.$ and $\left.\mathrm{H}-5^{\prime \prime}\right), 8.68(1 \mathrm{H}, \mathrm{s}$, $\mathrm{H}-8), 8.81\left(2 \mathrm{H}, \mathrm{m}, \mathrm{H}-2^{\prime \prime}\right.$ and $\left.\mathrm{H}-6{ }^{\prime \prime}\right) .{ }^{13} \mathrm{C}$ NMR (125 MHz, DMSO- $\left.d_{6}\right)$ $\delta 128.8,129.4,131.7,134.4,145.7,154.4,156.0,157.1,158.7$. LRMS $\left(\mathrm{ES}^{+}\right) \mathrm{m} / z 214.6[\mathrm{M}+\mathrm{H}]^{+}$.

2-Fluoro-6-(3-methoxyphenyl)-9H-purine (64). Deprotection of $57(0.24 \mathrm{~g}, 0.72 \mathrm{mmol})$ in accordance with general procedure $\mathrm{E}$ afforded the title compound $64(0.17 \mathrm{~g}, 98 \%)$ following repeated trituration of the precipitated solid with water; mp $237-238^{\circ} \mathrm{C}$; UV $\lambda_{\max }(\mathrm{EtOH}) 251 \mathrm{~nm}$. IR $\left(\mathrm{cm}^{-1}\right) 2837,1864,1587,1565 .{ }^{1} \mathrm{H}$ NMR $\left(300 \mathrm{MHz}, \mathrm{DMSO}-d_{6}\right) \delta 3.89\left(3 \mathrm{H}, \mathrm{s}, \mathrm{OCH}_{3}\right), 7.18\left(1 \mathrm{H}, \mathrm{s}, \mathrm{H}-2^{\prime \prime}\right)$, $7.50-7.55$ (1H, m, H-5"), 8.37 (1H, s, H-8), 8.45-8.49 (2H, m, H-4", H-6" $).{ }^{13} \mathrm{C}$ NMR (125 MHz, DMSO- $\left.d_{6}\right) \delta 55.3,114.2,117.6,121.8$, 130.0, 135.7, 146.3, 154.3, 157.2, 158.6, 159.4. LRMS $\left(\mathrm{ES}^{+}\right) \mathrm{m} / z 245.5$ $[\mathrm{M}+\mathrm{H}]^{+}$.

2-Fluoro-6-(4-methoxyphenyl)-9H-purine (65). Synthesized in accordance with general procedure $\mathrm{E}$ from $58(0.24 \mathrm{~g}, 0.72 \mathrm{mmol})$. Trituration of the precipitated solid with water furnished 65 as a paleyellow solid $(0.17 \mathrm{~g}, 95 \%)$; mp $212-214{ }^{\circ} \mathrm{C}$; UV $\lambda_{\max }(\mathrm{EtOH}) 204$ nm. IR $\left(\mathrm{cm}^{-1}\right) 2845,1586 .{ }^{1} \mathrm{H}$ NMR (300 MHz, DMSO-d $\left.d_{6}\right) \delta 3.88$ $\left(3 \mathrm{H}, \mathrm{s}, \mathrm{OCH}_{3}\right), 7.15-7.18\left(2 \mathrm{H}, \mathrm{d}, J=9.0 \mathrm{~Hz}, \mathrm{H}-3^{\prime \prime}\right.$ and $\left.\mathrm{H}-5^{\prime \prime}\right), 8.60$ $(1 \mathrm{H}, \mathrm{s}, \mathrm{H}-8), 8.79-8.81\left(2 \mathrm{H}, \mathrm{d}, J=9.0 \mathrm{~Hz}, \mathrm{H}-2^{\prime \prime}\right.$ and $\left.\mathrm{H}-6^{\prime \prime}\right) .{ }^{13} \mathrm{C}$ NMR (500 MHz, DMSO-d 6 ) $\delta 55.4,114.1,114.3,126.8,127.5,131.3$, 145.3, 157.1, 158.8, 162.2. LRMS $\left(\mathrm{ES}^{+}\right) \mathrm{m} / z$ 245.2 $[\mathrm{M}+\mathrm{H}]^{+}$.

6-(Biphenyl-3-yl)-2-fluoro-9H-purine (66). The title compound was prepared according to general procedure E from $59(0.27 \mathrm{~g}, 0.72$ $\mathrm{mmol})$. Repeated washing of the precipitated solid with water gave 66 $(0.20 \mathrm{~g}, 97 \%)$; mp $245-247^{\circ} \mathrm{C}$; UV $\lambda_{\max }(\mathrm{EtOH}) 349 \mathrm{~nm}$. IR $\left(\mathrm{cm}^{-1}\right)$ $1607,1568 .{ }^{1} \mathrm{H}$ NMR $\left(300 \mathrm{MHz}\right.$, DMSO- $\left.d_{6}\right) \delta 7.43-7.45(1 \mathrm{H}, \mathrm{d}, J=$ $\left.7.0 \mathrm{~Hz}, \mathrm{H}-6^{\prime \prime}\right), 7.54-7.58\left(1 \mathrm{H}, \mathrm{dd}, J=6.9,7.0 \mathrm{~Hz}, \mathrm{H}-5^{\prime \prime}\right), 7.74-7.81$ $(5 \mathrm{H}, \mathrm{m}, \mathrm{Ph}), 8.40\left(1 \mathrm{H}, \mathrm{s}, \mathrm{H}-2^{\prime \prime}\right), 8.80-8.85\left(1 \mathrm{H}, \mathrm{d}, J=6.9 \mathrm{~Hz}, \mathrm{H}-4^{\prime \prime}\right)$, $8.91(1 \mathrm{H}, \mathrm{s}, \mathrm{H}-8) .{ }^{13} \mathrm{C}$ NMR $\left(125 \mathrm{MHz}, \mathrm{DMSO}-d_{6}\right) \delta 126.8,127.7$, $127.9,128.2,129.1,129.5,130.0,135.0,139.7,140.7,146.1\left(\mathrm{~m}, J_{\mathrm{C}-\mathrm{F}}\right)$, 157.1, 158.7. LRMS $\left(\mathrm{ES}^{+}\right) \mathrm{m} / z$ 291.1 $[\mathrm{M}+\mathrm{H}]^{+}$.

6-(Benzo[d][1,3]dioxol-5-yl)-2-fluoro-9H-purine (67). Deprotection of $60(0.25 \mathrm{~g}, 0.72 \mathrm{mmol})$ was achieved following general procedure E. Trituration of the white solid that precipitated yielded 67 (0.16 g, 87\%); mp $165-167{ }^{\circ} \mathrm{C}$; UV $\lambda_{\max }(\mathrm{EtOH}) 351,292 \mathrm{~nm}$. IR $\left(\mathrm{cm}^{-1}\right)$ 2955, 2859, 1601, 1570, 1504. ${ }^{1} \mathrm{H}$ NMR (300 MHz, DMSO$\left.d_{6}\right) \delta 6.17\left(2 \mathrm{H}, \mathrm{s}, \mathrm{OCH}_{2} \mathrm{O}\right), 7.18-7.18\left(1 \mathrm{H}, \mathrm{d}, J=8.0 \mathrm{~Hz}, \mathrm{H}-5^{\prime \prime}\right), 8.28$ $\left(1 \mathrm{H}, \mathrm{s}, \mathrm{H}-2^{\prime \prime}\right), 8.48-8.51\left(1 \mathrm{H}, \mathrm{d}, J=8.0 \mathrm{~Hz}, \mathrm{H}-6^{\prime \prime}\right), 8.63(1 \mathrm{H}, \mathrm{s}, \mathrm{H}-8)$. ${ }^{13} \mathrm{C}$ NMR (125 MHz, DMSO- $\left.d_{6}\right) \delta 101.9,108.6,125.1,128.4,145.3$, 
$147.8,150.3,153.9\left(\mathrm{~d}, J_{\mathrm{C}-\mathrm{F}}=17.5 \mathrm{~Hz}\right), 156.0\left(\mathrm{~d}, J_{\mathrm{C}-\mathrm{F}}=150.0 \mathrm{~Hz}\right)$, 157.0, 158.6. LRMS $\left(\mathrm{ES}^{+}\right) \mathrm{m} / z$ 259.2 $[\mathrm{M}+\mathrm{H}]^{+}$.

6-(4-Dibenzofuranyl)-2-fluoro-9H-purine (68). The title compound was prepared in accordance with general procedure $\mathrm{E}$ from $61(0.28 \mathrm{~g}, 0.72 \mathrm{mmol})$ and purified by repeated washing with water to afford 68 as an off-white solid $(0.20 \mathrm{~g}, 94 \%)$; mp $266-268{ }^{\circ} \mathrm{C}$; UV $\lambda_{\max }(\mathrm{EtOH}) 250 \mathrm{~nm}$. IR $\left(\mathrm{cm}^{-1}\right)$ 3020, 2923, 2832, 1867, 1611, 1568. ${ }^{1} \mathrm{H}$ NMR $\left(300 \mathrm{MHz}\right.$, DMSO- $\left.d_{6}\right) \delta 7.45-7.48(1 \mathrm{H}, \mathrm{dd}, J=7.0,7.1 \mathrm{~Hz}$, $\left.\mathrm{H}-2^{\prime \prime}\right), 7.55-7.63\left(1 \mathrm{H}, \mathrm{dd}, J=7.9,8.0 \mathrm{~Hz}, \mathrm{H}-7^{\prime \prime}\right), 7.61-7.68(1 \mathrm{H}, \mathrm{dd}$, $\left.J=7.9,8.0 \mathrm{~Hz}, \mathrm{H}-8^{\prime \prime}\right), 7.76-7.79\left(1 \mathrm{H}, \mathrm{d}, J=8.0 \mathrm{~Hz}, \mathrm{H}-9^{\prime \prime}\right), 8.26-8.29$ $\left(1 \mathrm{H}, \mathrm{d}, J=7.1 \mathrm{~Hz}, \mathrm{H}-3^{\prime \prime}\right), 8.43-8.45\left(1 \mathrm{H}, \mathrm{d}, J=8.0 \mathrm{~Hz}, \mathrm{H}-6^{\prime \prime}\right), 8.55-$ $8.58\left(1 \mathrm{H}, \mathrm{d}, J=7.0 \mathrm{~Hz}, \mathrm{H}-1^{\prime \prime}\right), 8.78(1 \mathrm{H}, \mathrm{s}, \mathrm{H}-8) .{ }^{13} \mathrm{C}$ NMR $(500$ $\left.\mathrm{MHz}, \mathrm{DMSO}-d_{6}\right) \delta 112.1,121.3,123.0,123.5,124.0,125.0,128.0$, 130.0, 155.7. LRMS $\left(\mathrm{ES}^{+}\right) \mathrm{m} / z$ 305.1 $[\mathrm{M}+\mathrm{H}]^{+}$.

2-Fluoro-6-(thianthren-1-yl)-9H-purine (69). Synthesized following general procedure $\mathrm{E}$ from $62(0.32 \mathrm{~g}, 0.72 \mathrm{mmol})$ and purified by repeated trituration with water to give $\mathbf{6 9}(0.36 \mathrm{~g}, 93 \%)$; mp 166-168 ${ }^{\circ} \mathrm{C}$; UV $\lambda_{\max }(\mathrm{EtOH}) 250 \mathrm{~nm}$. IR $\left(\mathrm{cm}^{-1}\right) 3068,2817,2572,1609$, 1511. ${ }^{1} \mathrm{H}$ NMR $\left(300 \mathrm{MHz}\right.$, DMSO- $\left.d_{6}\right) \delta 7.21-7.29(1 \mathrm{H}, \mathrm{dd}, J=7.4$, $\left.7.5 \mathrm{~Hz}, \mathrm{H}-3^{\prime \prime}\right), 7.31-7.38$ (1H, dd, $\left.J=7.5,7.6 \mathrm{~Hz}, \mathrm{H}-7^{\prime \prime}\right), 7.39-7.46$ $\left(1 \mathrm{H}, \mathrm{d}, J=7.5 \mathrm{~Hz}, \mathrm{H}-9^{\prime \prime}\right), 7.49-7.55\left(1 \mathrm{H}, \mathrm{dd}, J=7.5,7.6 \mathrm{~Hz}, \mathrm{H}-8^{\prime \prime}\right)$, $7.58-7.64\left(1 \mathrm{H}, \mathrm{d}, J=7.5 \mathrm{~Hz}, \mathrm{H}-6^{\prime \prime}\right), 7.79\left(1 \mathrm{H}, \mathrm{d}, J=7.5 \mathrm{~Hz}, \mathrm{H}-2^{\prime \prime}\right)$, $7.88-7.91\left(1 \mathrm{H}, \mathrm{d}, J=7.4 \mathrm{~Hz}, \mathrm{H}-4^{\prime \prime}\right), 8.31(1 \mathrm{H}, \mathrm{s}, \mathrm{H}-8) .{ }^{13} \mathrm{C}$ NMR $\left(125 \mathrm{MHz}, \mathrm{DMSO}-d_{6}\right) \delta 124.6,127.5,128.2,128.4,128.7,128.9$, 130.5, 131.1, 134.7, 134.9, 156.4, 159.9, 160.5. LRMS $\left(\mathrm{ES}^{+}\right) \mathrm{m} / z 353.1$ $[\mathrm{M}+\mathrm{H}]^{+}$.

4-(6-Phenyl-9H-purin-2-ylamino)benzenesulfonamide (70). The title compound was synthesized according to general procedure $\mathrm{A}$ from 63 ( $0.10 \mathrm{~g}, 0.49 \mathrm{mmol})$. Purified by chromatography (silica; 50\% EtOAc:petrol) to give $\mathbf{7 0}$ as a white solid ( $80 \mathrm{mg}, 45 \%)$; mp 153-155 ${ }^{\circ} \mathrm{C}$; UV $\lambda_{\max }(\mathrm{EtOH}) 349,294 \mathrm{~nm}$. IR $\left(\mathrm{cm}^{-1}\right) 3313,2922,2852,1706$, $1589,1569,1524,1373,1144 .{ }^{1} \mathrm{H}$ NMR $\left(300 \mathrm{MHz}, \mathrm{DMSO}-d_{6}\right) \delta 7.17$ $\left(2 \mathrm{H}, \mathrm{br} \mathrm{s}, \mathrm{SO}_{2} \mathrm{NH}_{2}\right), 7.60\left(3 \mathrm{H}, \mathrm{m}, \mathrm{H}-3^{\prime \prime}, \mathrm{H}-4^{\prime \prime}\right.$ and $\left.\mathrm{H}-5^{\prime \prime}\right), 7.75-7.78$ $\left(2 \mathrm{H}, \mathrm{d}, J=8.5 \mathrm{~Hz}, \mathrm{H}-2^{\prime}\right.$ and $\left.\mathrm{H}-6^{\prime}\right), 8.03-8.06\left(2 \mathrm{H}, \mathrm{d}, J=8.5 \mathrm{~Hz}, \mathrm{H}-3^{\prime}\right.$ and $\left.\mathrm{H}-5^{\prime}\right) 8.37(1 \mathrm{H}, \mathrm{s}, \mathrm{H}-8), 8.94\left(2 \mathrm{H}, \mathrm{m}, \mathrm{H}-2^{\prime \prime}\right.$ and $\left.\mathrm{H}-6^{\prime \prime}\right), 9.91(1 \mathrm{H}$, br s, NH). ${ }^{13} \mathrm{C}$ NMR (125 MHz, DMSO-d $d_{6} \delta 117.2,125.4,126.5$, $128.5,129.3,130.8,135.4,135.8,142.6,144.3,153.0,154.6,155.3$. LRMS $\left(\mathrm{ES}^{+}\right) \mathrm{m} / z$ 367.2 $[\mathrm{M}+\mathrm{H}]^{+}$. HRMS calcd for $\mathrm{C}_{17} \mathrm{H}_{14} \mathrm{~N}_{6} \mathrm{O}_{2} \mathrm{~S}[\mathrm{M}$ $+\mathrm{H}]^{+}$367.0972, found 367.0974.

4-(6-(3-Methoxyphenyl)-9H-purin-2-ylamino)benzenesulfonamide (71). Prepared in accordance with general procedure A from $64(0.12 \mathrm{~g}, 0.49 \mathrm{mmol})$ and purified by chromatography (silica; 50\% EtOAc:petrol) to furnish 71 as a white solid (64 mg, 33\%); mp 288-290 ${ }^{\circ} \mathrm{C}$; UV $\lambda_{\max }(\mathrm{EtOH}) 294 \mathrm{~nm}$. IR $\left(\mathrm{cm}^{-1}\right)$ 3377, 3097, 3020, 2838, 1729, 1597, 1566, 1531, 1365, 1145. ${ }^{1} \mathrm{H}$ NMR $\left(300 \mathrm{MHz}, \mathrm{DMSO}-d_{6}\right) \delta 3.89\left(3 \mathrm{H}, \mathrm{s}, \mathrm{OCH}_{3}\right), 7.17(2 \mathrm{H}, \mathrm{s}$, $\left.\mathrm{SO}_{2} \mathrm{NH}_{2}\right), 7.18\left(1 \mathrm{H}, \mathrm{s}, \mathrm{H}-2^{\prime \prime}\right), 7.50-7.55\left(1 \mathrm{H}, \mathrm{m}, \mathrm{H}-5^{\prime \prime}\right), 7.74-7.77$ $\left(2 \mathrm{H}, \mathrm{d}, J=8.0 \mathrm{~Hz}, \mathrm{H}-2^{\prime}\right.$ and $\left.\mathrm{H}-6^{\prime}\right), 8.03-8.06\left(2 \mathrm{H}, \mathrm{d}, J=8.0 \mathrm{~Hz}, \mathrm{H}-3^{\prime}\right.$ and $\left.\mathrm{H}-5^{\prime}\right), 8.37(1 \mathrm{H}, \mathrm{s}, \mathrm{H}-8), 8.45-8.49\left(2 \mathrm{H}, \mathrm{m}, \mathrm{H}-4^{\prime \prime}\right.$ and $\left.\mathrm{H}-6^{\prime \prime}\right) .{ }^{13} \mathrm{C}$ NMR (125 MHz, DMSO- $\left.d_{6}\right) \delta 55.2,114.2,116.6,117.2,121.8,126.5$, 129.6, 135.4, 137.1, 144.3, 146.3, 155.2, 115.6, 159.3. LRMS $\left(\mathrm{ES}^{+}\right) \mathrm{m} /$ $z 397.3[\mathrm{M}+\mathrm{H}]^{+}$. HRMS calcd for $\mathrm{C}_{18} \mathrm{H}_{16} \mathrm{~N}_{6} \mathrm{O}_{3} \mathrm{~S}[\mathrm{M}+\mathrm{H}]^{+}$397.1077, found 397.1071 .

4-(6-(4-Methoxyphenyl)-9H-purin-2-ylamino)benzenesulfonamide (72). Synthesed following general procedure A from $65(0.12 \mathrm{~g}, 0.49 \mathrm{mmol})$ and isolated by chromatography (silica; 50\% EtOAc:petrol) to yield 72 as a white solid (90 mg, 46\%); mp 172-174 ${ }^{\circ} \mathrm{C}$; UV $\lambda_{\max }(\mathrm{EtOH}) 301,203 \mathrm{~nm}$. IR $\left(\mathrm{cm}^{-1}\right) 3322,2133$, $2005,1594,1533,1514,1352,1147 .{ }^{1} \mathrm{H}$ NMR $\left(300 \mathrm{MHz}\right.$, DMSO-d $\left.d_{6}\right)$ $\delta 3.88\left(3 \mathrm{H}, \mathrm{s}, \mathrm{OCH}_{3}\right), 7.17\left(2 \mathrm{H}\right.$, br s, $\left.\mathrm{SO}_{2} \mathrm{NH}_{2}\right), 7.18(2 \mathrm{H}, \mathrm{d}, J=8.5$ $\mathrm{Hz}, \mathrm{H}-3^{\prime \prime}$ and $\left.\mathrm{H}-5^{\prime \prime}\right), 7.74-7.77\left(2 \mathrm{H}, \mathrm{d}, J=8.0 \mathrm{~Hz}, \mathrm{H}-2^{\prime}\right.$ and $\left.\mathrm{H}-6^{\prime}\right)$, $8.02-8.05\left(2 \mathrm{H}, \mathrm{d}, J=8.0 \mathrm{~Hz}, \mathrm{H}-3^{\prime}\right.$ and $\left.\mathrm{H}-5^{\prime}\right), 8.32(1 \mathrm{H}, \mathrm{s}, \mathrm{H}-8)$, 8.84-8.87 (2H, d, $J=8.5 \mathrm{~Hz}, \mathrm{H}-2^{\prime \prime}$ and $\left.\mathrm{H}-6^{\prime \prime}\right) .{ }^{13} \mathrm{C} \mathrm{NMR}(125 \mathrm{MHz}$, DMSO- $\left.d_{6}\right) \delta 55.4,114.0,117.2,124.8,126.5,128.2,131.0,135.3$, 142.0, 144.4, 152.8, 154.3, 155.3, 161.5. LRMS $\left(\mathrm{ES}^{+}\right) \mathrm{m} / z 397.3[\mathrm{M}+$ $\mathrm{H}]^{+}$. HRMS calcd for $\mathrm{C}_{18} \mathrm{H}_{16} \mathrm{~N}_{6} \mathrm{O}_{3} \mathrm{~S}[\mathrm{M}+\mathrm{H}]^{+}$397.1077, found 397.1071.

4-((6-([1,1'-Biphenyl]-3-yl)-9H-purin-2-yl)amino)benzenesulfonamide (73). Treatment of $66(0.14 \mathrm{~g}, 0.49 \mathrm{mmol})$ in accordance with general procedure A with purification by chromatog- raphy (silica; EtOAc) afforded 73 as a white solid (46 mg, 21\%); mp 153-155 ${ }^{\circ} \mathrm{C}$; UV $\lambda_{\max }$ (EtOH) 349, $294 \mathrm{~nm}$. IR $\left(\mathrm{cm}^{-1}\right)$ 3313, 2922, 2852, 1706, 1589, 1569, 1524, 1373, 1144. ${ }^{1} \mathrm{H}$ NMR $(300 \mathrm{MHz}$, DMSO- $\left.d_{6}\right) \delta 7.18\left(1 \mathrm{H}, \mathrm{br} \mathrm{s}, \mathrm{SO}_{2} \mathrm{NH}_{2}\right), 7.39-7.50(1 \mathrm{H}, \mathrm{d}, J=7.0 \mathrm{~Hz}$, H-6"), 7.50-7.62 (1H, dd, $\left.J=7.0,7.1 \mathrm{~Hz}, \mathrm{H}-5^{\prime \prime}\right), 7.66-7.83(5 \mathrm{H}, \mathrm{m}$, $\mathrm{Ph}), 7.83-7.93\left(2 \mathrm{H}, \mathrm{d}, J=8.0 \mathrm{~Hz}, \mathrm{H}-2^{\prime}\right.$ and $\left.\mathrm{H}-6^{\prime}\right), 8.00-8.13(2 \mathrm{H}, \mathrm{d}$, $J=8.0 \mathrm{~Hz}, \mathrm{H}-3^{\prime}$ and $\left.\mathrm{H}-5^{\prime}\right), 8.35-8.40\left(1 \mathrm{H}, \mathrm{s}, \mathrm{H}-2^{\prime \prime}\right), 8.78-8.93(1 \mathrm{H}$, d, $\left.J=7.1 \mathrm{~Hz}, \mathrm{H}-4^{\prime \prime}\right), 9.18(1 \mathrm{H}, \mathrm{s}, \mathrm{H}-8), 10.00(1 \mathrm{H}, \mathrm{br} \mathrm{s}, \mathrm{NH})$. LRMS $\left(\mathrm{ES}^{+}\right) \mathrm{m} / z 367.2[\mathrm{M}+\mathrm{H}]^{+}$. HRMS calcd for $\mathrm{C}_{23} \mathrm{H}_{18} \mathrm{~N}_{6} \mathrm{O}_{2} \mathrm{~S}[\mathrm{M}+\mathrm{H}]^{+}$ 367.0972, found 367.0974. HPLC: >96\%, XSELECT column, retention time $7.48 \mathrm{~min}$, reverse phase, $\mathrm{H}_{2} \mathrm{O} / \mathrm{MeCN} / \mathrm{HCOOH}$ gradient run, $1 \mathrm{~mL} \mathrm{~min}^{-1}$.

4-(6-(Benzo[d][1,3]dioxol-5-yl)-9H-purin-2-ylamino)benzenesulfonamide (74). The title compound was prepared following general procedure A from $67(0.13 \mathrm{~g}, 0.49 \mathrm{mmol})$. Purification by chromatography (silica; EtOAc) gave 74 as a paleyellow solid (30 mg, 15\%); mp $284-286{ }^{\circ} \mathrm{C}$; UV $\lambda_{\max }(\mathrm{EtOH}) 352$, $295 \mathrm{~nm}$. IR $\left(\mathrm{cm}^{-1}\right) 3174,1573,1526,1408,1350,1153 .{ }^{1} \mathrm{H}$ NMR $\left(300 \mathrm{MHz}, \mathrm{DMSO}-d_{6}\right) \delta 6.14\left(2 \mathrm{H}, \mathrm{s}, \mathrm{OCH}_{2} \mathrm{O}\right), 7.15(2 \mathrm{H}, \mathrm{s}$, $\left.\mathrm{SO}_{2} \mathrm{NH}_{2}\right), 7.73-7.75\left(2 \mathrm{H}, \mathrm{d}, J=8.0, \mathrm{H}-2^{\prime}\right.$ and $\left.\mathrm{H}-6^{\prime}\right), 7.98-8.01(2 \mathrm{H}$, $\mathrm{d}, J=8.0 \mathrm{~Hz}, \mathrm{H}-3^{\prime}$ and $\left.\mathrm{H}-5^{\prime}\right), 8.31(1 \mathrm{H}, \mathrm{s}, \mathrm{H}-8), 8.37\left(1 \mathrm{H}, \mathrm{s}, \mathrm{H}-2^{\prime \prime}\right)$, $8.55\left(2 \mathrm{H}, \mathrm{m}, \mathrm{H}-5^{\prime \prime}\right.$ and $\left.\mathrm{H}-6^{\prime \prime}\right), 9.89(1 \mathrm{H}, \mathrm{br} \mathrm{s}, \mathrm{NH}) .{ }^{13} \mathrm{C}$ NMR $(125$ $\left.\mathrm{MHz}, \mathrm{DMSO}-d_{6}\right) \delta 101.6,108.4,108.6,117.2,124.7,126.5,129.9$, 133.2 , 135.5, 139.8, 142.2, 144.3, 147.6, 149.6, 151.9, 152.4, 154.4, 155.2. LRMS $\left(\mathrm{ES}^{+}\right) \mathrm{m} / z$ 443.3 $[\mathrm{M}+\mathrm{H}]^{+}$. HRMS calcd for $\mathrm{C}_{18} \mathrm{H}_{14} \mathrm{~N}_{6} \mathrm{O}_{4} \mathrm{~S}[\mathrm{M}+\mathrm{H}]^{+}$443.1285, found 443.1289.

4-(6-(4-Dibenzofuryl)-9H-purin-2-ylamino)benzenesulfonamide (75). Synthesized in accordance with general procedure A from $\mathbf{6 8}$ (0.15 g, $0.49 \mathrm{mmol}$ ). Purification by chromatography (silica; 50\% EtOAc:petrol) gave 75 as a white solid $(0.10 \mathrm{~g}, 47 \%)$; mp 213-215 ${ }^{\circ} \mathrm{C}$; UV $\lambda_{\max }$ (EtOH) 292, $250 \mathrm{~nm}$. IR $\left(\mathrm{cm}^{-1}\right)$ 3275, 3199, 3104, 2921, $2852,1714,1593,1574,1535,1500,1355,1146 .{ }^{1} \mathrm{H}$ NMR $(300 \mathrm{MHz}$, DMSO- $\left.d_{6}\right) \delta 7.16\left(2 \mathrm{H}, \mathrm{br} \mathrm{s}, \mathrm{SO}_{2} \mathrm{NH}_{2}\right), 7.44-7.53(1 \mathrm{H}, \mathrm{dd}, J=6.9,7.0$ $\left.\mathrm{Hz}, \mathrm{H}-2^{\prime \prime}\right), 7.54-7.68\left(2 \mathrm{H}, \mathrm{m}, \mathrm{H}-7^{\prime \prime}\right.$ and $\left.\mathrm{H}-8^{\prime \prime}\right), 7.68-7.74(1 \mathrm{H}, \mathrm{d}, J=$ $\left.8.0 \mathrm{~Hz}, \mathrm{H}-9^{\prime \prime}\right), 7.69-7.79\left(2 \mathrm{H}, \mathrm{d}, J=8.0 \mathrm{~Hz}, \mathrm{H}-2^{\prime}\right.$ and $\left.\mathrm{H}-6^{\prime}\right), 8.18-$ $8.26\left(2 \mathrm{H}, \mathrm{d}, J=8.0 \mathrm{~Hz}, \mathrm{H}-3^{\prime}\right.$ and $\left.\mathrm{H}-5^{\prime}\right), 8.23-8.32(1 \mathrm{H}, \mathrm{d}, J=6.9 \mathrm{~Hz}$, H-3"), 8.38 (1H, s, H-8), 8.38-8.44 (1H, d, J = 7.5 Hz, H-6" ), 8.49$8.58\left(1 \mathrm{H}, \mathrm{d}, J=7.0 \mathrm{~Hz}, \mathrm{H}-1^{\prime \prime}\right), 10.07(1 \mathrm{H}$, br s, NH), $13.22(1 \mathrm{H}, \mathrm{br} \mathrm{s}$, $\mathrm{NH}) .{ }^{13} \mathrm{C}$ NMR $\left(125 \mathrm{MHz}\right.$, DMSO- $\left.d_{6}\right) \delta 111.5,117.3,121.0,121.3$, $122.9,123.1,123.2,123.4,124.9,126.4,128.0,130.0,135.4,143.0$, 144.4, 152.2, 153.0, 154.4, 155.3, 155.7. LRMS $\left(\mathrm{ES}^{+}\right) \mathrm{m} / z$ 457.3 $[\mathrm{M}+$ $\mathrm{H}]^{+}$. HRMS calcd for $\mathrm{C}_{23} \mathrm{H}_{16} \mathrm{~N}_{6} \mathrm{O}_{3} \mathrm{~S}[\mathrm{M}+\mathrm{H}]^{+} 457.1077$, found 457.1075 .

6-(4-Thianthren-1-yl)-9H-purin-2-ylamino)benzenesulfonamide (76). Synthesized following general procedure A from (69) (0.17 g, $0.49 \mathrm{mmol}$ ), and purified by chromatography (silica; 50\% EtOAc:petrol) to furnish 76 as an orange solid (81 mg, 33\%); mp 170-172 ${ }^{\circ} \mathrm{C}$; UV $\lambda_{\max }(\mathrm{EtOH}) 339,294,250 \mathrm{~nm}$. IR $\left(\mathrm{cm}^{-1}\right)$ 3317, 2922, 2851, $1706,1588,1524,1371,1146 .{ }^{1} \mathrm{H}$ NMR $\left(300 \mathrm{MHz}, \mathrm{DMSO}-d_{6}\right) \delta 7.16$ $\left(2 \mathrm{H}, \mathrm{s}, \mathrm{SO}_{2} \mathrm{NH}_{2}\right), 7.21-7.28\left(1 \mathrm{H}, \mathrm{dd}, J=7.4,7.5 \mathrm{~Hz}, \mathrm{H}-3^{\prime \prime}\right), 7.30-$ $7.36\left(1 \mathrm{H}, \mathrm{dd}, J=7.5,7.6 \mathrm{~Hz}, \mathrm{H}-7^{\prime \prime}\right), 7.38-7.43(1 \mathrm{H}, \mathrm{d}, J=7.5 \mathrm{~Hz}, \mathrm{H}-$ $\left.9^{\prime \prime}\right), 7.48-7.53\left(1 \mathrm{H}, \mathrm{dd}, J=7.5,7.6 \mathrm{~Hz}, \mathrm{H}-8^{\prime \prime}\right), 7.58-7.63(1 \mathrm{H}, \mathrm{d}, J=$ $\left.7.5 \mathrm{~Hz}, \mathrm{H}-6^{\prime \prime}\right), 7.73\left(2 \mathrm{H}, \mathrm{d}, J=8.5 \mathrm{~Hz}, \mathrm{H}-2^{\prime}\right.$ and $\left.\mathrm{H}-6^{\prime}\right), 7.77-7.80$ $\left(1 \mathrm{H}, \mathrm{d}, J=7.4 \mathrm{~Hz}, \mathrm{H}-2^{\prime \prime}\right), 7.88-7.91\left(1 \mathrm{H}, \mathrm{d}, J=7.5 \mathrm{~Hz}, \mathrm{H}-4^{\prime \prime}\right), 8.06$ $\left(2 \mathrm{H}, \mathrm{d}, J=8.5 \mathrm{~Hz}, \mathrm{H}-3^{\prime}\right.$ and $\left.\mathrm{H}-5^{\prime}\right), 8.34(1 \mathrm{H}, \mathrm{s}, \mathrm{H}-8)$. LRMS $\left(\mathrm{ES}^{+}\right) \mathrm{m} /$ $z 505.2[\mathrm{M}+\mathrm{H}]^{+}$. HRMS calcd for $\mathrm{C}_{23} \mathrm{H}_{16} \mathrm{~N}_{6} \mathrm{O}_{2} \mathrm{~S}_{3}[\mathrm{M}+\mathrm{H}]^{+}$ 505.0570, found 505.0573.

7-Chloro-3-(4-methoxybenzyl)-3H-imidazo[4,5-b]pyridin-5amine (78). To a stirred solution of $77(0.04 \mathrm{~g}, 0.24 \mathrm{mmol})$ in anhydrous DMF $(1 \mathrm{~mL})$ was added anhydrous $\mathrm{K}_{2} \mathrm{CO}_{3}(36 \mathrm{mg}, 0.26$ $\mathrm{mmol}$ ) and 4-methoxybenzyl chloride $(34 \mu \mathrm{L}, 0.25 \mathrm{mmol})$. The reaction mixture was stirred under $\mathrm{N}_{2}$ at $65{ }^{\circ} \mathrm{C}$ for $18 \mathrm{~h}$, and the solvent was removed under reduced pressure. The residual solid was redissolved in EtOAc $(30 \mathrm{~mL})$ and washed with brine $(3 \times 30 \mathrm{~mL})$, and the combined organic fractions were dried $\left(\mathrm{Na}_{2} \mathrm{SO}_{4}\right)$ and the solvent was removed under reduced pressure. Purification by chromatography (silica; 40\% EtOAc:petrol) afforded 78 as a lightyellow solid (29 mg, 42\%); $R_{\mathrm{f}} 0.21$ (40\% EtOAc:petrol); mp $105^{\circ} \mathrm{C}$; UV $\lambda_{\max }(\mathrm{EtOH}) 311 \mathrm{~nm}$. IR $\left(\mathrm{cm}^{-1}\right) 3431,3312,3198,3022,2940$, 2798, 1571, 1513. ${ }^{1} \mathrm{H}$ NMR (500 MHz, DMSO- $\left.d_{6}\right) \delta 3.71(3 \mathrm{H}, \mathrm{s}$, 
$\left.\mathrm{OCH}_{3}\right), 5.22\left(2 \mathrm{H}, \mathrm{s}, \mathrm{CH}_{2}\right), 6.24\left(2 \mathrm{H}, \mathrm{br} \mathrm{s}, \mathrm{NH}_{2}\right), 6.48(1 \mathrm{H}, \mathrm{s}, \mathrm{H}-5)$, $6.88\left(2 \mathrm{H}, \mathrm{d}, J=8.7 \mathrm{~Hz}, \mathrm{H}-2^{\prime \prime}\right.$ and $\left.\mathrm{H}-6^{\prime \prime}\right), 7.23\left(2 \mathrm{H}, \mathrm{d}, J=8.8 \mathrm{~Hz}, \mathrm{H}-3^{\prime \prime}\right.$ and $\left.\mathrm{H}-5^{\prime \prime}\right), 8.07$ (1H, s, H-2). ${ }^{13} \mathrm{C}$ NMR (125 MHz, DMSO- $\left.d_{6}\right) \delta 45.4$ ( $\left.\mathrm{Ar}-\mathrm{CH}_{2}-\mathrm{Ar}\right), 55.1\left(\mathrm{OCH}_{3}\right), 103.8$ (Ar-C), 114.0 (Ar-C), $124.4(\mathrm{Ar}-$ C), 128.7 (Ar-C), 129.2 (Ar-C), 133.9 (Ar-C), 140.5 (Ar-C), 146.6 (Ar-C), 157.4 (Ar-C), 158.7 (Ar-C). HRMS calcd for $\mathrm{C}_{14} \mathrm{H}_{14} \mathrm{ClN}_{4} \mathrm{O}$ $[\mathrm{M}+\mathrm{H}]^{+} 289.0851$, found 289.0855 .

7-(Cyclohexylmethoxy)-3-(4-methoxybenzyl)-3H-imidazo[4,5-b]pyridin-5-amine (79). Synthesized following general method $\mathrm{C}$ from $78(0.172 \mathrm{~g}, 0.60 \mathrm{mmol})$. The product was isolated by chromatography (silica; $80 \%$ EtOAc:petrol) to furnish 79 as a colorless oil $(0.16$ g, $0.45 \mathrm{mmol}, 75 \%) ; R_{\mathrm{f}} 0.47$ (20\% EtOAc:petrol); UV $\lambda_{\max }$ (EtOH) $271 \mathrm{~nm}$. IR $\left(\mathrm{cm}^{-1}\right) 3311,2954,2899,2163,1672,1598,1516 .{ }^{1} \mathrm{H}$ NMR $\left(500 \mathrm{MHz}, \mathrm{DMSO}-d_{6}\right) \delta 0.90-1.87(11 \mathrm{H}, \mathrm{m}$, cyclohexyl), 3.71 $\left(3 \mathrm{H}, \mathrm{s}, \mathrm{OCH}_{3}\right), 4.00\left(2 \mathrm{H}, \mathrm{d}, J=6.7 \mathrm{~Hz}, \mathrm{OCH}_{2}\right), 5.17\left(2 \mathrm{H}, \mathrm{br} \mathrm{s}, \mathrm{NH}_{2}\right)$, $5.24\left(2 \mathrm{H}, \mathrm{s}, \mathrm{CH}_{2}\right), 5.90(1 \mathrm{H}, \mathrm{s}, \mathrm{H}-5), 6.87\left(2 \mathrm{H}, \mathrm{d}, J=8.7 \mathrm{~Hz}, \mathrm{H}-2^{\prime \prime}\right.$ and $\left.\mathrm{H}-6^{\prime \prime}\right), 7.20\left(2 \mathrm{H}, \mathrm{d}, J=8.8 \mathrm{~Hz}, \mathrm{H}-3^{\prime \prime}\right.$ and $\left.\mathrm{H}-5^{\prime \prime}\right), 7.82(1 \mathrm{H}, \mathrm{s}, \mathrm{H}-2)$. ${ }^{13} \mathrm{C}$ NMR $\left(125 \mathrm{MHz}, \mathrm{DMSO}-d_{6}\right) \delta 25.2\left(\mathrm{CH}_{2}\right), 26.0\left(\mathrm{CH}_{2}\right), 29.1$ $\left(\mathrm{CH}_{2}\right), 37.0(\mathrm{CH}), 45.0\left(\mathrm{Ar}-\mathrm{CH}_{2}\right), 55.1\left(\mathrm{CH}_{3}\right), 73.3\left(\mathrm{CH}_{2}\right), 87.5(\mathrm{Ar}-$ C), 113.9 (Ar-C), 118.2 (Ar-C), 128.7 (Ar-C), 129.7 (Ar-C), 137.9 (Ar-C), 147.4 (Ar-C), 157.8 (Ar-C), 158.5 (Ar-C), 158.6 (Ar-C). HRMS calcd for $\mathrm{C}_{21} \mathrm{H}_{27} \mathrm{~N}_{4} \mathrm{O}_{2}[\mathrm{M}+\mathrm{H}]^{+} 367.2129$, found 367.2132.

7-(Cyclohexylmethoxy)-3H-imidazo[4,5-b]pyridin-5-amine (80). A solution of $79(0.035 \mathrm{~g}, 0.10 \mathrm{mmol})$ in TFA $(1 \mathrm{~mL})$ was heated under reflux for $2 \mathrm{~h}$, and the cooled solution was neutralized with saturated aqueous $\mathrm{NaHCO}_{3}(25 \mathrm{~mL})$. The reaction mixture was extracted with EtOAc $(3 \times 30 \mathrm{~mL})$, and the combined organic fraction was dried $\left(\mathrm{MgSO}_{4}\right)$ and evaporated under reduced pressure. Purification by chromatography (silica; $10 \% \mathrm{MeOH}: \mathrm{DCM}$ ) to furnish 80 as a white solid $(16.5 \mathrm{mg}, 70 \%) ; \mathrm{mp} 99-102{ }^{\circ} \mathrm{C}$; UV $\lambda_{\max }(\mathrm{EtOH})$ 293, $244 \mathrm{~nm}$. IR $\left(\mathrm{cm}^{-1}\right)$ 3447, 3323, 3187, 2923, 2850, 2795, 2026, 1591, 1527. ${ }^{1} \mathrm{H}$ NMR $\left(500 \mathrm{MHz}, \mathrm{CDCl}_{3}\right) \delta 1.03-2.00(11 \mathrm{H}, \mathrm{m}$, cyclohexyl), $4.03\left(2 \mathrm{H}, \mathrm{d}, J=6.1 \mathrm{~Hz}, \mathrm{OCH}_{2}\right), 4.37\left(2 \mathrm{H}, \mathrm{br} \mathrm{s}, \mathrm{NH}_{2}\right)$, $5.91(1 \mathrm{H}, \mathrm{s}, \mathrm{H}-5), 7.77(1 \mathrm{H}, \mathrm{s}, \mathrm{H}-2), 9.89(1 \mathrm{H}, \mathrm{br} \mathrm{s}, \mathrm{NH}) .{ }^{13} \mathrm{C}$ NMR $\left(125 \mathrm{MHz}, \mathrm{CDCl}_{3}\right) \delta 25.7,26.4,29.8,37.5,74.4,88.1,157.3$. HRMS calcd for $\mathrm{C}_{13} \mathrm{H}_{19} \mathrm{~N}_{4} \mathrm{O}[\mathrm{M}+\mathrm{H}]^{+}$247.1553, found 247.1556.

7-(Cyclohexylmethoxy)-5-iodo-3-(4-methoxybenzyl)-3H-imidazo[4,5-b]pyridine (81). A solution of $79(0.112 \mathrm{~g}, 0.31 \mathrm{mmol})$ in THF (4 $\mathrm{mL})$ containing diiodomethane $(123 \mu \mathrm{L}, 1.50 \mathrm{mmol}), \mathrm{CuI}(59 \mathrm{mg}$, $0.31 \mathrm{mmol})$, and isoamyl nitrite $(187 \mu \mathrm{L}, 0.92 \mathrm{mmol})$ was heated to reflux for $4 \mathrm{~h}$, and the dark-brown solution was filtered (Celite) and extracted with EtOAc $(2 \times 20 \mathrm{~mL})$. The combined organic fraction was washed sequentially with aqueous sodium thiosulfate solution $(10 \%, 2 \times 40 \mathrm{~mL})$ and brine $(40 \mathrm{~mL})$ and dried $\left(\mathrm{MgSO}_{4}\right)$, and the solvent was removed in vacuo. Purification by chromatography (silica; $30 \%$ EtOAc:petrol) afforded 81 as a yellow oil $(64 \mathrm{mg}, 44 \%) ; R_{\mathrm{f}} 0.19$ (30\% EtOAc:petrol); UV $\lambda_{\max }$ (EtOH) $262 \mathrm{~nm}$. IR $\left(\mathrm{cm}^{-1}\right) 3333,2951$, 2891, 2122, 1672, 1584, 1521. ${ }^{1} \mathrm{H}$ NMR $\left.(500 \mathrm{MHz}, \text { DMSO-d })_{6}\right) \delta$ 0.98-1.89 (11H, m, cyclohexyl), $3.71\left(3 \mathrm{H}, \mathrm{s}, \mathrm{OCH}_{3}\right), 4.19(2 \mathrm{H}, \mathrm{d}, J=$ $\left.6.5 \mathrm{~Hz}, \mathrm{OCH}_{2}\right), 5.17\left(2 \mathrm{H}, \mathrm{s}, \mathrm{CH}_{2}\right), 6.90\left(2 \mathrm{H}, \mathrm{d}, J=8.7 \mathrm{~Hz}, \mathrm{H}-2^{\prime \prime}\right.$ and $\left.\mathrm{H}-6^{\prime \prime}\right), 7.18(1 \mathrm{H}, \mathrm{s}, \mathrm{H}-5), 7.26\left(2 \mathrm{H}, \mathrm{d}, J=8.8 \mathrm{~Hz}, \mathrm{H}-3^{\prime \prime}\right.$ and $\left.\mathrm{H}-5^{\prime \prime}\right)$, $8.31(1 \mathrm{H}, \mathrm{s}, \mathrm{H}-2) .{ }^{13} \mathrm{C}$ NMR $\left(125 \mathrm{MHz}\right.$, DMSO-d $\left.d_{6}\right) \delta 25.1\left(\mathrm{CH}_{2}\right)$, $25.9\left(\mathrm{CH}_{2}\right), 28.9\left(\mathrm{CH}_{2}\right), 37.0(\mathrm{CH}), 45.8\left(\mathrm{Ar}-\mathrm{CH}_{2}\right), 55.1\left(\mathrm{CH}_{3}\right), 74.4$ $\left(\mathrm{CH}_{2}\right), 112.0$ (Ar-C), 112.7 (Ar-C), 114.1 (Ar-C), 124.6 (Ar-C), 128.8 (Ar-C), 129.0 (Ar-C), 142.6 (Ar-C), 148.5 ( $\mathrm{Ar}-\mathrm{C}), 157.0$ (Ar-C), 158.8 (Ar-C). HRMS calcd for $\mathrm{C}_{21} \mathrm{H}_{25} \mathrm{IN}_{3} \mathrm{O}_{2}[\mathrm{M}+\mathrm{H}]^{+}$478.0986, found 478.0980 .

4-((7-(Cyclohexylmethoxy)-3-(4-methoxybenzyl)-3H-imidazo[4,5b]pyridin-5-yl) amino)benzenesulfonamide (82). To a solution of 81 $(0.048 \mathrm{~g}, 0.10 \mathrm{mmol})$ in anhydrous $\mathrm{MeCN}(2 \mathrm{~mL})$ was added sulfanilamide $(19 \mathrm{mg}, 0.11 \mathrm{mmol})$, anhydrous $\mathrm{K}_{2} \mathrm{CO}_{3}(28 \mathrm{mg}, 0.20$ $\mathrm{mmol}), \mathrm{Pd}_{2}(\mathrm{dba})_{3}(2.3 \mathrm{mg}, 0.004 \mathrm{mmol})$, and XPhos (1.9 mg, 0.004 $\mathrm{mmol}$ ). The reaction mixture was degassed under $\mathrm{N}_{2}$ for $30 \mathrm{~min}$ and stirred at $80{ }^{\circ} \mathrm{C}$ for $2 \mathrm{~h}$. The cooled solution was filtered (Celite) extracted with EtOAc $(2 \times 20 \mathrm{~mL})$, and the combined organic extract was washed sequentially with aqueous $\mathrm{HCl}(1 \mathrm{M}, 20 \mathrm{~mL})$, saturated aqeuous $\mathrm{NaHCO}_{3}(20 \mathrm{~mL})$, and brine $(30 \mathrm{~mL})$, and dried $\left(\mathrm{Na}_{2} \mathrm{SO}_{4}\right)$, and the volatiles were evaporated in vacuo. Purification by chromatography (silica; 70\% EtOAc:petrol) yielded 82 as a white solid (23 mg, 43\%); $R_{\mathrm{f}} 0.59$ (70\% EtOAc:petrol); mp $142{ }^{\circ} \mathrm{C}$; UV $\lambda_{\max }$
(EtOH) $257 \mathrm{~nm} . \mathrm{IR}\left(\mathrm{cm}^{-1}\right)$ 3329, 2933, 2874, 2158, 1662, 1588, 1501. ${ }^{1} \mathrm{H}$ NMR $\left(500 \mathrm{MHz}, \mathrm{CDCl}_{3}\right) \delta 1.00-1.97(11 \mathrm{H}, \mathrm{m}$, cyclohexyl), $3.79\left(3 \mathrm{H}, \mathrm{s}, \mathrm{OCH}_{3}\right), 4.01\left(2 \mathrm{H}, \mathrm{d}, J=6.1 \mathrm{~Hz}, \mathrm{OCH}_{2}\right), 4.76(2 \mathrm{H}, \mathrm{br} \mathrm{s}$, $\left.\mathrm{NH}_{2}\right), 5.28\left(2 \mathrm{H}, \mathrm{s}, \mathrm{CH}_{2}\right), 6.17(1 \mathrm{H}, \mathrm{s}, \mathrm{H}-5), 6.79(1 \mathrm{H}, \mathrm{br} \mathrm{s}, \mathrm{NH}), 6.88$ $\left(2 \mathrm{H}, \mathrm{d}, J=8.8 \mathrm{~Hz}, \mathrm{H}-2^{\prime \prime}\right.$ and $\left.\mathrm{H}-6^{\prime \prime}\right), 7.24\left(2 \mathrm{H}, \mathrm{d}, J=8.7 \mathrm{~Hz}, \mathrm{H}-3^{\prime \prime}\right.$ and $\left.\mathrm{H}-5^{\prime \prime}\right), 7.61\left(2 \mathrm{H}, J=8.8 \mathrm{~Hz}, \mathrm{H}-2^{\prime}\right.$ and $\left.\mathrm{H}-6^{\prime}\right), 7.81(2 \mathrm{H}, J=8.8 \mathrm{~Hz}, \mathrm{H}-$ $3^{\prime}$ and $\left.\mathrm{H}-5^{\prime}\right), 8.11(1 \mathrm{H}, \mathrm{s}, \mathrm{H}-2) .{ }^{13} \mathrm{C}$ NMR $\left(125 \mathrm{MHz}, \mathrm{CDCl}_{3}\right) \delta 25.7$ $\left(\mathrm{CH}_{2}\right), 26.4\left(\mathrm{CH}_{2}\right), 29.8\left(\mathrm{CH}_{2}\right), 37.6\left(\mathrm{CH}_{2}\right), 46.9(\mathrm{CH}), 55.4\left(\mathrm{CH}_{2}\right)$, $74.8\left(\mathrm{OCH}_{3}\right), 90.7\left(\mathrm{OCH}_{2}\right), 114.3(\mathrm{Ar}-\mathrm{C}), 117.1$ (Ar-C), $128.0(\mathrm{Ar}-$ C), 129.1 (Ar-C), 139.7 (Ar-C), 145.5 (Ar-C), 152.1 (Ar-C). HRMS calcd for $\mathrm{C}_{27} \mathrm{H}_{32} \mathrm{~N}_{5} \mathrm{O}_{4} \mathrm{~S}[\mathrm{M}+\mathrm{H}]^{+} 522.2170$, found 522.2166 .

4-((7-(Cyclohexylmethoxy)-3H-imidazo[4,5-b]pyridin-5-yl)amino) benzenesulfonamide (83). A solution of $\mathbf{8 2}(0.022 \mathrm{~g}, 0.042 \mathrm{mmol})$ in TFA $(1 \mathrm{~mL})$ was heated under reflux for $2 \mathrm{~h}$, and the cooled solution was neutralized with saturated aqueous $\mathrm{NaHCO}_{3}(25 \mathrm{~mL})$. The reaction mixture was extracted with EtOAc $(3 \times 30 \mathrm{~mL})$, and the combined organic fraction was dried $\left(\mathrm{MgSO}_{4}\right)$ and evaporated under reduced pressure. The title compound was purified by preparative TLC on silica, using $5 \% \mathrm{MeOH}: \mathrm{DCM}$ as eluent, to afford 83 as a white solid (16.7 mg, 99\%); $R_{\mathrm{f}} 0.25$ (5\% MeOH:DCM); mp 165-166 ${ }^{\circ} \mathrm{C}$; UV $\lambda_{\max }(\mathrm{EtOH}) 324,274 \mathrm{~nm}$. IR $\left(\mathrm{cm}^{-1}\right) 3332,2925,2852,2164$, $1677,1583,1500 .{ }^{1} \mathrm{H}$ NMR $\left(500 \mathrm{MHz}, \mathrm{MeOD}-d_{4}\right) \delta 1.07-2.05(11 \mathrm{H}$, $\mathrm{m}$, cyclohexyl), $4.03\left(2 \mathrm{H}, \mathrm{d}, J=6.2 \mathrm{~Hz}, \mathrm{OCH}_{2}\right), 6.33(1 \mathrm{H}, \mathrm{s}, \mathrm{H}-5)$, $7.77\left(2 \mathrm{H}, \mathrm{d}, J=8.9 \mathrm{~Hz}, \mathrm{H}-2^{\prime}\right.$ and $\left.\mathrm{H}-6^{\prime}\right), 7.92\left(2 \mathrm{H}, \mathrm{d}, J=8.8 \mathrm{~Hz}, \mathrm{H}-3^{\prime}\right.$ and $\left.\mathrm{H}-5^{\prime}\right), 7.96(1 \mathrm{H}, \mathrm{s}, \mathrm{H}-2) .{ }^{13} \mathrm{C}$ NMR $\left(125 \mathrm{MHz}, \mathrm{CDCl}_{3}\right) \delta 26.9$ $\left(\mathrm{CH}_{2}\right), 27.6\left(\mathrm{CH}_{2}\right), 30.8\left(\mathrm{CH}_{2}\right), 38.9(\mathrm{CH}), 74.9\left(\mathrm{CH}_{2} \mathrm{O}\right), 90.9(\mathrm{Ar}-$ C), 117.9 (Ar-C), 128.2 (Ar-C). HRMS calcd for $\mathrm{C}_{19} \mathrm{H}_{24} \mathrm{~N}_{5} \mathrm{O}_{3} \mathrm{~S}[\mathrm{M}+$ $\mathrm{H}]^{+}$522.2170, found 522.2166. HPLC: $>98 \%$, XSELECT column, retention time $5.47 \mathrm{~min}$, reverse phase, $\mathrm{H}_{2} \mathrm{O} / \mathrm{MeCN} / \mathrm{HCOOH}$ gradient run, $1 \mathrm{~mL} \mathrm{~min}{ }^{-1}$.

7-(Cyclohexylmethoxy)-3H-[1,2,3]triazolo[4,5-d]pyrimidin-5amine (86). Prepared in accordance with general procedure $\mathrm{C}$ from 84 $(1.50 \mathrm{~g}, 8.82 \mathrm{mmol})$. Precipitation from aqueous $\mathrm{AcOH}$, and repeated washing with water gave 86 a pale-orange solid (1.52 g, 70\%); mp 214-216 ${ }^{\circ} \mathrm{C}$; UV $\lambda_{\max }(\mathrm{EtOH}) 285 \mathrm{~nm}$. IR $\left(\mathrm{cm}^{-1}\right) 3494,3378,2925$, 2780, 2651, 2362, 2329, 2115, 2085, 2013, 1906, 1628, 1608, 1578, 1488. ${ }^{1} \mathrm{H}$ NMR $\left(500 \mathrm{MHz}, \mathrm{DMSO}-d_{6}\right) \delta 1.03-1.89(11 \mathrm{H}, \mathrm{m}$, cyclohexyl), $4.30\left(2 \mathrm{H}, \mathrm{d}, J=6.5 \mathrm{~Hz}, \mathrm{OCH}_{2}\right), 6.91\left(2 \mathrm{H}, \mathrm{br} \mathrm{s}, \mathrm{NH}_{2}\right)$, $15.28\left(1 \mathrm{H}\right.$, br s, NH) ${ }^{13} \mathrm{C}$ NMR $(125 \mathrm{MHz}$, DMSO-d $) \delta 25.1\left(\mathrm{CH}_{2}\right)$, $25.9\left(\mathrm{CH}_{2}\right), 29.1\left(\mathrm{CH}_{2}\right), 36.6(\mathrm{CH}), 71.2\left(\mathrm{CH}_{2}\right), 162.2(\mathrm{Ar}-\mathrm{C})$. HRMS calcd for $\mathrm{C}_{11} \mathrm{H}_{17} \mathrm{~N}_{6} \mathrm{O}[\mathrm{M}+\mathrm{H}]^{+} 249.1458$, found 249.1461 .

4-(Cyclohexylmethoxy)-1H-pyrazolo[3,4-d]pyrimidin-6-amine (87). Prepared according to the general procedure C from 85 ( $0.125 \mathrm{~g}$, $0.74 \mathrm{mmol}$ ). Purification by chromatography (silica; $10 \%$ $\mathrm{MeOH}: \mathrm{DCM})$ gave 87 as an off white crystalline solid $(68 \mathrm{mg}$, $37 \%) ; R_{\mathrm{f}} 0.70$ (10\% MeOH:DCM), mp $193-195{ }^{\circ} \mathrm{C}$; UV $\lambda_{\max }$ (EtOH) $275 \mathrm{~nm}$. IR $\left(\mathrm{cm}^{-1}\right)$ 3498, 3353, 3232, 3121, 2960, 2921, 2851, 2750, 2667, 2158, 2038, 1902, 1794, 1633, 1578, 1497, 1469, 1441. ${ }^{1} \mathrm{H}$ NMR (500 MHz, DMSO- $\left.d_{6}\right) \delta 0.98-1.82(11 \mathrm{H}, \mathrm{m}$, cyclohexyl), $4.21\left(2 \mathrm{H}, \mathrm{d}, J=6.5 \mathrm{~Hz}, \mathrm{OCH}_{2}\right), 6.55\left(2 \mathrm{H}, \mathrm{br} \mathrm{s}, \mathrm{NH}_{2}\right)$, $7.77(1 \mathrm{H}, \mathrm{s}, \mathrm{CH}), 12.78(1 \mathrm{H}, \mathrm{br} \mathrm{s}, \mathrm{NH}) .{ }^{13} \mathrm{C}$ NMR $(125 \mathrm{MHz}$, DMSO- $\left.d_{6}\right) \delta 25.2\left(\mathrm{CH}_{2}\right), 26.0\left(\mathrm{CH}_{2}\right), 29.1\left(\mathrm{CH}_{2}\right), 36.7(\mathrm{CH}), 70.5$ $\left(\mathrm{CH}_{2}\right), 95.4(\mathrm{Ar}-\mathrm{C}), 131.6$ ( $\left.\mathrm{Ar}-\mathrm{C}\right), 158.9$ (Ar-C), 162.0 (Ar-C), 163.4 (Ar-C). HRMS calcd for $\mathrm{C}_{12} \mathrm{H}_{18} \mathrm{~N}_{5} \mathrm{O}[\mathrm{M}+\mathrm{H}]^{+}$248.1506, found 248.1508

5-Chloro-7-(cyclohexylmethoxy)-3H-[1,2,3]triazolo [4,5-d]pyrimidine (88). To a solution of $\mathrm{LiCl}(34 \mathrm{mg}, 0.80 \mathrm{mmol})$ in DMA $(0.5 \mathrm{~mL})$ at $0{ }^{\circ} \mathrm{C}$ was added $86(0.05 \mathrm{~g}, 0.20 \mathrm{mmol})$, followed by isoamyl nitrite $(61 \mu \mathrm{L}, 0.30 \mathrm{mmol})$ and $\operatorname{SOCl}_{2}(16 \mu \mathrm{L}, 0.22 \mathrm{mmol})$. The reaction mixture was stirred for $1 \mathrm{~h}$ and allowed to warm to ambient temperature over $18 \mathrm{~h}$. After addition of saturated aqueous $\mathrm{NaHCO}_{3}(15 \mathrm{~mL})$, the reaction mixture was extracted with EtOAc (3 $\times 15 \mathrm{~mL})$, and the combined organic layers were washed with brine (2 $\times 30 \mathrm{~mL})$ and dried $\left(\mathrm{Na}_{2} \mathrm{SO}_{4}\right)$. Evaporation of the solvent under reduced pressure furnished $\mathbf{8 8}$ as a brown oil (54 $\mathrm{mg},>99 \%)$, which was used directly without further purification.

6-Chloro-4-(cyclohexylmethoxy)-1H-pyrazolo[3,4-d]pyrimidine (89). To a solution of $\mathrm{LiCl}(69 \mathrm{mg}, 1.62 \mathrm{mmol})$ in DMA $(1 \mathrm{~mL})$ at 0 ${ }^{\circ} \mathrm{C}$ was added $87(0.100 \mathrm{~g}, 0.40 \mathrm{mmol})$, followed by isoamyl nitrite $(123 \mu \mathrm{L}, 0.61 \mathrm{mmol})$ and $\operatorname{SOCl}_{2}(32 \mu \mathrm{L}, 0.45 \mathrm{mmol})$. The reaction 
mixture was stirred at room temperature for $18 \mathrm{~h}$, cooled, and EtOAc $(45 \mathrm{~mL})$ was added. The resultant solution was washed sequentially with aqueous saturated $\mathrm{NaHCO}_{3}$ solution $(45 \mathrm{~mL})$ and brine $(2 \times 45$ $\mathrm{mL})$, dried $\left(\mathrm{Na}_{2} \mathrm{SO}_{4}\right)$, and the volatiles were evaporated under reduced pressure. The product was purified by chromatography (silica; $5 \%$ $\mathrm{MeOH}: \mathrm{DCM})$ to give 89 as a yellow solid $(65 \mathrm{mg}, 60 \%) ; R_{\mathrm{f}} 0.32(5 \%$ $\mathrm{MeOH}: \mathrm{DCM}), \mathrm{mp} 152-153^{\circ} \mathrm{C}$; UV $\lambda_{\text {max }}(\mathrm{EtOH}) 252 \mathrm{~nm}$. IR $\left(\mathrm{cm}^{-1}\right)$ $3148,2922,2851,1677,1579,1443 .{ }^{1} \mathrm{H}$ NMR $\left(500 \mathrm{MHz}, \mathrm{CDCl}_{3}\right) \delta$ 0.96-1.95 (11H, m, cyclohexyl), $4.39\left(2 \mathrm{H}, \mathrm{d}, J=6.0 \mathrm{~Hz}, \mathrm{OCH}_{2}\right), 8.09$ $(1 \mathrm{H}, \mathrm{s}, \mathrm{CH}), 11.19(1 \mathrm{H}, \mathrm{br} \mathrm{s}, \mathrm{NH}) .{ }^{13} \mathrm{C}$ NMR $\left(125 \mathrm{MHz}\right.$, DMSO- $\left.d_{6}\right)$ $\delta 25.2\left(\mathrm{CH}_{2}\right), 26.0\left(\mathrm{CH}_{2}\right), 29.1\left(\mathrm{CH}_{2}\right), 36.7(\mathrm{CH}), 70.5\left(\mathrm{CH}_{2} \mathrm{O}\right), 95.4$ (Ar-C), $131.6\left(\mathrm{C}^{1}-\mathrm{H}\right), 158.9$ (Ar-C), 162.0 (Ar-C), 163.4 (Ar-C). HRMS calcd for $\mathrm{C}_{12} \mathrm{H}_{16} \mathrm{ClN}_{4} \mathrm{O}[\mathrm{M}+\mathrm{H}]^{+}$267.1007, found 267.1012.

4-((7-(Cyclohexylmethoxy)-3H-[1,2,3]triazolo[4,5-d]pyrimidin-5yl)amino) benzenesulfonamide (90). The title compound was prepared following general procedure A from 88 (0.054 g, 0.20 $\mathrm{mmol}$ ) and isolated by chromatography (silica; 30\% EtOAc:petrol) to give the title compound 90 as a white solid ( $28 \mathrm{mg}, 35 \%) ; R_{\mathrm{f}} 0.18$ (70\% EtOAc:petrol); mp 217-219 ${ }^{\circ} \mathrm{C}$; UV $\lambda_{\max }$ (EtOH) $310 \mathrm{~nm}$. IR $\left(\mathrm{cm}^{-1}\right) 3254,3121,2924,2851,2363,2342,2199,2140,1992,19118$, $1700,1582,1534,1492,1447 .{ }^{1} \mathrm{H}$ NMR $\left(500 \mathrm{MHz}, \mathrm{DMSO}-d_{6}\right) \delta$ 1.04-1.92 (11H, m, cyclohexyl), $4.36\left(2 \mathrm{H}, \mathrm{d}, J=6.8 \mathrm{~Hz}, \mathrm{OCH}_{2}\right), 7.18$ $\left(2 \mathrm{H}\right.$, br s, $\left.\mathrm{SO}_{2} \mathrm{NH}_{2}\right), 7.72\left(2 \mathrm{H}, \mathrm{d}, J=8.8 \mathrm{~Hz}, \mathrm{H}-2^{\prime}\right.$ and $\left.\mathrm{H}-6^{\prime}\right), 7.99$ $\left(2 \mathrm{H}, \mathrm{d}, J=9.0 \mathrm{~Hz}, \mathrm{H}-3^{\prime}\right.$ and $\left.\mathrm{H}-5^{\prime}\right), 9.92(1 \mathrm{H}, \mathrm{br} \mathrm{s}, \mathrm{NH}) 13.34(1 \mathrm{H}, \mathrm{br}$ s, $\mathrm{NH}) .{ }^{13} \mathrm{C}$ NMR $\left(125 \mathrm{MHz}, \mathrm{DMSO}-d_{6}\right) \delta 25.1\left(\mathrm{CH}_{2}\right), 29.1\left(\mathrm{CH}_{2}\right)$, $35.9(\mathrm{CH}), 118.0$ (Ar-C), 126.4 (Ar-C). HRMS calcd for $\mathrm{C}_{17} \mathrm{H}_{22} \mathrm{~N}_{7} \mathrm{O}_{3} \mathrm{~S}[\mathrm{M}+\mathrm{H}]^{+}$404.1499, found 404.1500. HPLC: $>99.5 \%$, XTerra column, retention time $11.64 \mathrm{~min}$, reverse phase, $\mathrm{H}_{2} \mathrm{O}$ /

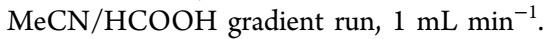

4-((4-(Cyclohexylmethoxy)-1H-pyrazolo[3,4-d]pyrimidin-6-yl)amino)benzenesulfonamide (91). The title compound was prepared by general procedure A from $89(0.042 \mathrm{~g}, 0.18 \mathrm{mmol})$. Chromatography (silica; 70\% EtOAc:petrol) afforded 91 as a white solid (20 mg, 28\%); $R_{\mathrm{f}} 0.21$ (70\% EtOAc:petrol), mp 268-269 ${ }^{\circ} \mathrm{C}$; UV $\lambda_{\max }(\mathrm{EtOH}) 304 \mathrm{~nm}$. IR $\left(\mathrm{cm}^{-1}\right) 3367,3333,3269,2922,2853$, 2162, 2012, 1691, 1658, 1624, 1583, 1551, 1524. ${ }^{1} \mathrm{H}$ NMR $(500 \mathrm{MHz}$, DMSO- $\left.d_{6}\right) \delta 1.04-1.92(11 \mathrm{H}, \mathrm{m}$, cyclohexyl $), 4.36(2 \mathrm{H}, \mathrm{d}, J=6.8 \mathrm{~Hz}$, $\left.\mathrm{OCH}_{2}\right), 7.18\left(2 \mathrm{H}, \mathrm{br} \mathrm{s}, \mathrm{SO}_{2} \mathrm{NH}_{2}\right), 7.72\left(2 \mathrm{H}, \mathrm{d}, J=8.8 \mathrm{~Hz}, \mathrm{H}-2^{\prime}\right.$ and H-6'), $7.98(1 \mathrm{H}$, br s, $\mathrm{CH}), 7.99\left(2 \mathrm{H}, \mathrm{d}, J=9.0 \mathrm{~Hz}, \mathrm{H}-3^{\prime}\right.$ and $\left.\mathrm{H}-5^{\prime}\right)$, $9.92(1 \mathrm{H}$, br s, $\mathrm{NH}) 13.34(1 \mathrm{H}$, br s, $\mathrm{NH}) .{ }^{13} \mathrm{C}$ NMR $(125 \mathrm{MHz}$, DMSO-d $\left.d_{6}\right) \delta 25.4\left(\mathrm{CH}_{2}\right), 26.5\left(\mathrm{CH}_{2}\right), 29.5\left(\mathrm{CH}_{2}\right), 37.1(\mathrm{CH}), 71.5$ $\left(\mathrm{CH}_{2}\right)$ 118.6 (Ar-C), 136.6 (Ar-C), 144.0 (Ar-C). HRMS calcd for $\mathrm{C}_{18} \mathrm{H}_{23} \mathrm{~N}_{6} \mathrm{O}_{3} \mathrm{~S}[\mathrm{M}+\mathrm{H}]^{+}$403.1547, found 403.1547. HPLC: $>98.5 \%$, XTerra column, retention time $11.92 \mathrm{~min}$, reverse phase, $\mathrm{H}_{2} \mathrm{O}$ /

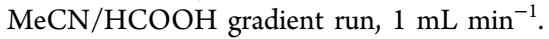

4-(Cyclohexylmethoxy)-7H-pyrrolo[2,3-d]pyrimidin-2-amine (93). Prepared in accordance with general method C from 92 ( $0.15 \mathrm{~g}$, 0.89 mmol), with heating under microwave irradiation $\left(170{ }^{\circ} \mathrm{C}, 5 \mathrm{~h}\right)$. The product was isolated by chromatography (silica; $10 \% \mathrm{MeOH}: \mathrm{DCM}$ ) to yield 93 as a white crystalline solid ( $37 \mathrm{mg}, 17 \%) ; R_{\mathrm{f}} 0.55(10 \%$ MeOH:DCM); mp 216.3-217.3 ${ }^{\circ} \mathrm{C}$; UV $\lambda_{\max }(\mathrm{EtOH}) 221 \mathrm{~nm}$. IR $\left(\mathrm{cm}^{-1}\right)$ 3494, 3362, 3117, 2922, 2851, 2164, 2045, 1968, 1617, 1585, 1499, 1478. ${ }^{1} \mathrm{H}$ NMR (500 MHz, DMSO- $\left.d_{6}\right) \delta 0.98-1.83(11 \mathrm{H}, \mathrm{m}$, cyclohexyl), $4.15\left(2 \mathrm{H}, \mathrm{d}, J=6.5, \mathrm{OCH}_{2}\right), 5.91\left(2 \mathrm{H}, \mathrm{br} \mathrm{s}, \mathrm{NH}_{2}\right), 6.17$ $(1 \mathrm{H}, \mathrm{dd}, J=1.9$ and $3.5, \mathrm{CH}), 6.79(1 \mathrm{H}, \mathrm{dd}, J=2.2$ and $3.5, \mathrm{HNCH})$, $10.97(1 \mathrm{H}$, br s, $\mathrm{NH}) .{ }^{13} \mathrm{C}$ NMR $\left(125 \mathrm{MHz}\right.$, DMSO- $\left.d_{6}\right) \delta 25.2\left(\mathrm{CH}_{2}\right)$, $26.0\left(\mathrm{CH}_{2}\right), 29.3\left(\mathrm{CH}_{2}\right), 36.8(\mathrm{CH}), 69.9\left(\mathrm{CH}_{2} \mathrm{O}\right), 96.9\left(\mathrm{C}^{1}-\mathrm{H}\right), 97.9$ (Ar-C), $119.1\left(\mathrm{C}^{2}-\mathrm{H}\right), 155.0$ (Ar-C), 159.3 (Ar-C), 162.7 (Ar-C). HRMS calcd for $\mathrm{C}_{13} \mathrm{H}_{19} \mathrm{~N}_{4} \mathrm{O}[\mathrm{M}+\mathrm{H}]^{+} 247.1553$, found 247.1556.

4-Chloro-7-((2-(trimethylsilyl)ethoxy)methyl)-7H-pyrrolo[2,3-d]pyrimidin-2-amine (94). $\mathrm{NaH}(60 \%$ in mineral oil, $26 \mathrm{mg}, 0.65$ $\mathrm{mmol})$ was added dropwise to a solution of $92(0.10 \mathrm{~g}, 0.59 \mathrm{mmol})$ in anhydrous $\mathrm{MeCN}(3 \mathrm{~mL})$ at $-30{ }^{\circ} \mathrm{C}$, and the reaction mixture was stirred for $45 \mathrm{~min}$. 2-(Trimethylsilyl)ethoxymethyl chloride (90\%, 122 $\mu \mathrm{L}, 0.62 \mathrm{mmol}$ ) was added dropwise, and the reaction mixture was stirred for a further $3 \mathrm{~h}$ at room temperature. After cooling and addition of water $(20 \mathrm{~mL})$, the resultant solution was extracted with EtOAc $(20 \mathrm{~mL})$, and the combined organic fractions were washed with brine $(3 \times 20 \mathrm{~mL})$, dried $\left(\mathrm{Na}_{2} \mathrm{SO}_{4}\right)$, and the solvent was removed in vacuo. Purification by chromatography (silica; $15 \%$ EtOAc:petrol) gave 94 as a yellow solid (130 mg, 74\%); $R_{\mathrm{f}} 0.41$ (15\% EtOAc:petrol); mp 63-64 ${ }^{\circ} \mathrm{C}$; UV $\lambda_{\max }(\mathrm{EtOH}) 317,234 \mathrm{~nm}$. IR $\left(\mathrm{cm}^{-1}\right) 3423,3318$, 3212, 3096, 2952, 1631, 1608, 1546, 1493. ${ }^{1} \mathrm{H}$ NMR $(500 \mathrm{MHz}$, $\left.\mathrm{CDCl}_{3}\right) \delta-0.04\left(9 \mathrm{H}, \mathrm{s},\left(\mathrm{CH}_{3}\right)_{3}\right), 0.91(2 \mathrm{H}, \mathrm{t}, J=8.0 \mathrm{~Hz}$, $\left.\mathrm{OCH}_{2} \mathrm{CH}_{2} \mathrm{Si}\right), 3.81\left(2 \mathrm{H}, \mathrm{t}, J=8.3 \mathrm{~Hz}, \mathrm{OCH}_{2} \mathrm{CH}_{2} \mathrm{Si}\right), 4.96(2 \mathrm{H}, \mathrm{br}$ s, $\left.\mathrm{NH}_{2}\right), 5.44\left(2 \mathrm{H}, \mathrm{s}, \mathrm{CH}_{2}\right), 6.43(1 \mathrm{H}, \mathrm{d}, J=3.7 \mathrm{~Hz}, \mathrm{CH}), 6.99(1 \mathrm{H}, \mathrm{d}$, $J=3.7 \mathrm{~Hz}, \mathrm{NCH}) .{ }^{13} \mathrm{C} \mathrm{NMR}\left(125 \mathrm{MHz}\right.$, DMSO- $\left.d_{6}\right) \delta 0.0\left(\mathrm{Si}\left(\mathrm{CH}_{3}\right)_{3}\right)$, $19.2\left(\mathrm{CH}_{2} \mathrm{CH}_{2} \mathrm{Si}\right), 67.7\left(\mathrm{OCH}_{2} \mathrm{CH}_{2}\right), 74.3\left(\mathrm{NCH}_{2} \mathrm{O}\right), 102.2\left(\mathrm{C}^{5} \mathrm{H}\right)$, 112.2 (Ar-C), $127.0\left(\mathrm{C}^{6} \mathrm{H}\right), 154.2$ (Ar-C), 155.6 (Ar-C), 160.2 (Ar-C). HRMS calcd for $\mathrm{C}_{12} \mathrm{H}_{20} \mathrm{ClN}_{4} \mathrm{OSi}[\mathrm{M}+\mathrm{H}]^{+} 299.1089$, found 299.1090 .

4-(Cyclohexylmethoxy)-7-((2-(trimethylsilyl)ethoxy)methyl)-7Hpyrrolo[2,3-d]pyrimidin-2-amine (95). Synthesized following general procedure C from $94(0.30 \mathrm{~g}, 1.01 \mathrm{mmol})$, except using THF as solvent. The title compound was isolated by chromatography (silica; $15 \%$ EtOAc:petrol) to furnish 95 as a yellow solid (296 mg, 78\%); $R_{\mathrm{f}}$ 0.17 (15\% EtOAc:petrol); mp 88-90 ${ }^{\circ} \mathrm{C}$; UV $\lambda_{\max }(\mathrm{EtOH}) 286,260$, $223 \mathrm{~nm} . \mathrm{IR}\left(\mathrm{cm}^{-1}\right) 3346,3222,2926,2852,1647,1602 .{ }^{1} \mathrm{H}$ NMR $\left(500 \mathrm{MHz}, \mathrm{CDCl}_{3}\right) \delta-0.05\left(9 \mathrm{H}, \mathrm{s},\left(\mathrm{CH}_{3}\right)_{3}\right), 0.91(2 \mathrm{H}, \mathrm{t}, J=8.3 \mathrm{~Hz}$, $\left.\mathrm{OCH}_{2} \mathrm{CH}_{2} \mathrm{Si}\right), 1.01-1.91(11 \mathrm{H}, \mathrm{m}$ cyclohexyl $), 3.51(2 \mathrm{H}, \mathrm{t}, J=8.3 \mathrm{~Hz}$, $\left.\mathrm{OCH}_{2} \mathrm{CH}_{2} \mathrm{Si}\right), 4.21\left(2 \mathrm{H}, \mathrm{d}, J=6.5 \mathrm{~Hz}, \mathrm{OCH}_{2}\right), 4.68\left(2 \mathrm{H}, \mathrm{br} \mathrm{s}, \mathrm{NH}_{2}\right)$, $5.41\left(2 \mathrm{H}, \mathrm{s}, \mathrm{CH}_{2}\right), 6.40(1 \mathrm{H}, \mathrm{d}, J=3.7 \mathrm{~Hz}, \mathrm{CH}), 6.82(1 \mathrm{H}, \mathrm{d}, J=3.7$ $\mathrm{Hz}, \mathrm{NCH}) .{ }^{13} \mathrm{C}$ NMR $\left(125 \mathrm{MHz}\right.$, DMSO- $\left.d_{6}\right) \delta 0.0\left(\mathrm{Si}\left(\mathrm{CH}_{3}\right)_{3}\right), 19.2$ $\left(\mathrm{CH}_{2} \mathrm{CH}_{2} \mathrm{Si}\right), 27.2\left(\mathrm{CH}_{2}\right), 28.0\left(\mathrm{CH}_{2}\right), 31.3\left(\mathrm{CH}_{2}\right), 38.8(\mathrm{CH}), 67.4$ $\left(\mathrm{OCH}_{2} \mathrm{CH}_{2}\right), 72.6\left(\mathrm{CH}_{2}\right), 74.1\left(\mathrm{NCH}_{2} \mathrm{O}\right), 101.2\left(\mathrm{C}^{5} \mathrm{H}\right), 123.6\left(\mathrm{C}^{6} \mathrm{H}\right)$, 160.6 (Ar-C), 165.4 (Ar-C). HRMS calcd for $\mathrm{C}_{19} \mathrm{H}_{33} \mathrm{~N}_{4} \mathrm{O}_{2} \mathrm{Si}[\mathrm{M}+\mathrm{H}]^{+}$ 377.2367 , found 377.2367 .

2-Chloro-4-(cyclohexylmethoxy)-7-((2-(trimethylsilyl)ethoxy)methyl)-7H-pyrrolo[2,3-d]pyrimidine (96). To a solution of $\mathrm{LiCl}$ in THF (0.5 M, $2.13 \mathrm{~mL}, 1.1 \mathrm{mmol})$ was added $95(0.10 \mathrm{~g}, 0.27 \mathrm{mmol})$, and the reaction mixture was cooled to $0{ }^{\circ} \mathrm{C}$. Isoamyl nitrite $(81 \mu \mathrm{L}$, $0.40 \mathrm{mmol})$ and $\mathrm{SOCl}_{2}(21.3 \mu \mathrm{L}, 0.29 \mathrm{mmol})$ were added, and the solution was stirred at room temperature for $5 \mathrm{~h}$. The reaction mixture was added to a solution of aqueous $\mathrm{NH}_{4} \mathrm{Cl}(2 \mathrm{M}, 30 \mathrm{~mL})$, extracted with EtOAc $(2 \times 30 \mathrm{~mL})$, and the combined organic layers were washed with water $(3 \times 30 \mathrm{~mL})$, dried $\left(\mathrm{Na}_{2} \mathrm{SO}_{4}\right)$, and evaporated in vacuo. Purification by chromatography (silica; $15 \%$ EtOAc:petrol) gave 96 as a clear oil (60 mg, 56\%); $R_{\mathrm{f}} 0.45$ (15\% EtOAc:petrol); UV $\lambda_{\max }(\mathrm{EtOH}) 270 \mathrm{~nm} . \mathrm{IR}\left(\mathrm{cm}^{-1}\right) 2924,2852,1750,1661,1588,1558$, 1506. ${ }^{1} \mathrm{H}$ NMR $\left(500 \mathrm{MHz}, \mathrm{DMSO}-d_{6}\right) \delta-0.09\left(9 \mathrm{H}, \mathrm{s},\left(\mathrm{CH}_{3}\right)_{3}\right), 0.82$ $\left(2 \mathrm{H}, \mathrm{t}, J=8.1 \mathrm{~Hz}, \mathrm{OCH}_{2} \mathrm{CH}_{2} \mathrm{Si}\right), 0.95-1.89(11 \mathrm{H}, \mathrm{m}$ cyclohexyl), 3.50 $\left(2 \mathrm{H}, \mathrm{t}, J=8.1 \mathrm{~Hz}, \mathrm{OCH}_{2} \mathrm{CH}_{2} \mathrm{Si}\right), 4.28\left(2 \mathrm{H}, \mathrm{d}, J=6.0 \mathrm{~Hz}, \mathrm{OCH}_{2}\right), 5.52$ $\left(2 \mathrm{H}, \mathrm{s}, \mathrm{CH}_{2}\right), 6.61(1 \mathrm{H}, \mathrm{d}, J=3.6 \mathrm{~Hz}, \mathrm{CH}), 7.55(1 \mathrm{H}, \mathrm{d}, J=3.5 \mathrm{~Hz}$, $\mathrm{NCH}) .{ }^{13} \mathrm{C}$ NMR $\left(125 \mathrm{MHz}, \mathrm{DMSO}-d_{6}\right) \delta 0.0\left(\mathrm{Si}\left(\mathrm{CH}_{3}\right)_{3}\right), 18.5$ $\left(\mathrm{CH}_{2} \mathrm{CH}_{2} \mathrm{Si}\right), 26.6\left(\mathrm{CH}_{2}\right), 30.5\left(\mathrm{CH}_{2}\right) 31.8\left(\mathrm{CH}_{2}\right), 38.8(\mathrm{CH}), 67.1$ $\left(\mathrm{OCH}_{2} \mathrm{CH}_{2}\right), 73.3\left(\mathrm{CH}_{2}\right), 74.2\left(\mathrm{NCH}_{2} \mathrm{O}\right), 100.4\left(\mathrm{C}^{5} \mathrm{H}\right), 126.4\left(\mathrm{C}^{6} \mathrm{H}\right)$, 129.7 (Ar-C). HRMS calcd for $\mathrm{C}_{19} \mathrm{H}_{31} \mathrm{ClN}_{3} \mathrm{O}_{2} \mathrm{Si}[\mathrm{M}+\mathrm{H}]^{+}$396.1869, found 396.1872 .

4-((4-(Cyclohexylmethoxy)-7-((2-(trimethylsilyl)ethoxy)methyl)7H-pyrrolo[2,3-d]pyrimidin-2-yl)amino)benzenesulfonamide (97). To a degassed solution of $96(0.050 \mathrm{~g}, 0.13 \mathrm{mmol})$ in $\mathrm{MeCN}(2$ $\mathrm{mL}$ ) was added sulfanilamide $\left(24 \mathrm{mg}, 0.14 \mathrm{mmol}\right.$ ), anhydrous $\mathrm{K}_{2} \mathrm{CO}_{3}$ $(35 \mathrm{mg}, 0.25 \mathrm{mmol}), \mathrm{Pd}_{2}(\mathrm{dba})_{3}\left(3 \mathrm{mg}, 5.0 \times 10^{-3} \mathrm{mmol}\right)$, and XPhos $\left(2.4 \mathrm{mg}, 5.0 \times 10^{-3} \mathrm{mmol}\right)$, and the reaction mixture was stirred under $\mathrm{N}_{2}$ at $80{ }^{\circ} \mathrm{C}$ for $2 \mathrm{~h}$. The cooled mixture was filtered (Celite), diluted with EtOAc $(20 \mathrm{~mL})$, and washed sequentially with aqueous $\mathrm{HCl}$ solution $(1 \mathrm{M}, 20 \mathrm{~mL})$, saturated aqueous $\mathrm{NaHCO}_{3}$ solution $(20 \mathrm{~mL})$, and brine $(30 \mathrm{~mL})$. After drying $\left(\mathrm{Na}_{2} \mathrm{SO}_{4}\right)$, volatiles were removed under reduced pressure and the product was isolated by chromatography (silica; 50\% EtOAc:petrol) to give 97 as a yellow oil $(33 \mathrm{mg}$, $49 \%) ; R_{\mathrm{f}} 0.63$ (50\% EtOAc:petrol); UV $\lambda_{\max }(\mathrm{EtOH}) 269 \mathrm{~nm}$. IR $\left(\mathrm{cm}^{-1}\right) 3644,3342,2924,2853,1586,1528,1488 .{ }^{1} \mathrm{H}$ NMR (500 $\left.\mathrm{MHz}, \mathrm{CDCl}_{3}\right) \delta-0.08\left(9 \mathrm{H}, \mathrm{s}, \mathrm{Si}\left(\mathrm{CH}_{3}\right)_{3}\right), 0.88-0.95(2 \mathrm{H}, \mathrm{t}, J=8.36$ $\left.\mathrm{Hz}, \mathrm{OCH}_{2} \mathrm{CH}_{2} \mathrm{Si}\right) 1.04-1.93(11 \mathrm{H}, \mathrm{m}$, cyclohexyl), $3.56(2 \mathrm{H}, \mathrm{t}, J=3.4$ $\left.\mathrm{Hz}, \mathrm{OCH}_{2} \mathrm{CH}_{2} \mathrm{Si}\right) 4.29\left(2 \mathrm{H}, \mathrm{d}, J=6.5 \mathrm{~Hz}, \mathrm{OCH}_{2}\right), 4.72(2 \mathrm{H}, \mathrm{br} \mathrm{s}$, $\left.\mathrm{NH}_{2}\right), 5.52\left(2 \mathrm{H}, \mathrm{s}, \mathrm{CH}_{2}\right), 6.49(1 \mathrm{H}, \mathrm{d}, J=3.5 \mathrm{~Hz}, \mathrm{CH}), 6.96(1 \mathrm{H}, \mathrm{d}, J$ $=3.6 \mathrm{~Hz}, \mathrm{NCH}), 7.20(1 \mathrm{H}, \mathrm{br} \mathrm{s}, \mathrm{NH}), 7.87\left(4 \mathrm{H}\right.$, ap s, $\mathrm{H}-2^{\prime}, \mathrm{H}-3^{\prime}, \mathrm{H}-5^{\prime}$ and $\left.\mathrm{H}-6^{\prime}\right) .{ }^{13} \mathrm{C}$ NMR $\left(125 \mathrm{MHz}, \mathrm{CDCl}_{3}\right) \delta 0.0\left(\mathrm{SiCH}_{3}\right), 15.6$ $\left(\mathrm{CH}_{2} \mathrm{Si}\right), 27.2\left(\mathrm{CH}_{2}\right), 27.9\left(\mathrm{CH}_{2}\right), 31.3\left(\mathrm{CH}_{2}\right), 38.8(\mathrm{CH}), 67.8$ $\left(\mathrm{CH}_{2}\right), 73.2\left(\mathrm{CH}_{2}\right), 74.5\left(\mathrm{CH}_{2}\right), 101.5$ (Ar-C), 101.9 (Ar-C), 118.8 (Ar-C), 125.0 (Ar-C), 129.3 (Ar-C), 146.2 (Ar-C). HRMS calcd for 
$\mathrm{C}_{25} \mathrm{H}_{38} \mathrm{~N}_{5} \mathrm{O}_{4} \mathrm{SSi}[\mathrm{M}+\mathrm{H}]^{+}$532.2408, found 532.2406. Note: Not all carbons are visible.

4-((4-(Cyclohexylmethoxy)-7H-pyrrolo[2,3-d]pyrimidin-2-yl)amino)benzenesulfonamide (98). A solution of 97 (0.040 g, $7.5 \times$ $\left.10^{-2} \mathrm{mmol}\right)$ in TFA $(1.5 \mathrm{~mL})$ was heated under reflux for $10 \mathrm{~min}$, and the cooled solution was neutralized with saturated aqueous $\mathrm{NaHCO}_{3}$ solution. The aqueous solution was extracted with EtOAc $(2 \times 30$ $\mathrm{mL}$ ), and the solvent was evaporated under reduced pressure. The residual yellow solid was redissolved in $\mathrm{MeCN}: \mathrm{H}_{2} \mathrm{O}(1: 1,8 \mathrm{~mL})$, the $\mathrm{pH}$ of the solution was adjusted to 10 with dilute aqueous ammonia $(28 \%)$, and the solution was stirred for $1 \mathrm{~h}$ at room temperature and evaporated in vacuo. Isolation by chromatography (silica; $70 \%$ EtOAc:petrol) to give 98 as a white solid (12 mg, 40\%); $R_{\mathrm{f}} 0.39$ (70\% EtOAc:petrol); mp 251.2-252.0 ${ }^{\circ} \mathrm{C}$; UV $\lambda_{\max }(\mathrm{EtOH}) 312 \mathrm{~nm}$. IR $\left(\mathrm{cm}^{-1}\right) 3374,3322,2058,2924,2856,2012,1615,1595,1579 .{ }^{1} \mathrm{H}$ NMR (500 MHz, DMSO- $\left.d_{6}\right) \delta 1.07-1.95(11 \mathrm{H}, \mathrm{m}$, cyclohexyl), 4.31 $\left(2 \mathrm{H}, \mathrm{d}, J=6.3 \mathrm{~Hz}, \mathrm{OCH}_{2}\right), 6.34(1 \mathrm{H}, \mathrm{dd}, J=2.1$ and $2.1 \mathrm{~Hz}, \mathrm{CH})$, $7.05(1 \mathrm{H}, \mathrm{dd}, J=2.2$ and $2.2 \mathrm{~Hz}, \mathrm{HNCH}), 7.13\left(2 \mathrm{H}, \mathrm{br} \mathrm{s}, \mathrm{SO}_{2} \mathrm{NH}_{2}\right)$, $7.68\left(2 \mathrm{H}, \mathrm{d}, J=8.9 \mathrm{~Hz}, \mathrm{H}-2^{\prime}\right.$ and $\left.\mathrm{H}^{-} 6^{\prime}\right) 7.99\left(2 \mathrm{H}, \mathrm{d}, J=8.8 \mathrm{~Hz}, \mathrm{H}-3^{\prime}\right.$ and $\left.\mathrm{H}-5^{\prime}\right), 9.57(1 \mathrm{H}$, br s, $\mathrm{NH}), 11.54(1 \mathrm{H}, \mathrm{br} \mathrm{s}, \mathrm{NH}) .{ }^{13} \mathrm{C} \mathrm{NMR}(125$ $\left.\mathrm{MHz}, \mathrm{DMSO}-d_{6}\right) \delta 25.2\left(\mathrm{CH}_{2}\right), 26.0\left(\mathrm{CH}_{2}\right), 29.3\left(\mathrm{CH}_{2}\right), 36.8(\mathrm{CH})$, $70.6\left(\mathrm{CH}_{2}\right), 98.2\left(\mathrm{C}^{5} \mathrm{H}\right), 116.9(\mathrm{Ar}-\mathrm{C}), 126.4\left(\mathrm{C}^{6} \mathrm{H}\right), 134.9$ (Ar-C), 144.6 (Ar-C), 146.4 (Ar-C), 153.6 (Ar-C), 154.4 (Ar-C), 162.4 (ArC). HRMS calcd for $\mathrm{C}_{19} \mathrm{H}_{24} \mathrm{~N}_{5} \mathrm{O}_{3} \mathrm{~S}[\mathrm{M}+\mathrm{H}]^{+} 402.1594$, found 402.1596. HPLC: $>99.5 \%$, XSELECT column, retention time 8.45 min, reverse phase, $\mathrm{H}_{2} \mathrm{O} / \mathrm{MeCN} / \mathrm{HCOOH}$ gradient run, $1 \mathrm{~mL} \mathrm{~min}^{-1}$.

7-Chloro-5-cyclohexylmethoxyimidazo[1,2-a]pyrimidine (100). Synthesized in accordance with general procedure C from 99 (1.0 g, $5.4 \mathrm{mmol})$. The product was purified by precipitation with glacial acetic acid, and repeated washing with water, to afford 100 as a paleorange powder (1.16 g, 81\%); $R_{\mathrm{f}} 0.23$ (60\% EtOAc:petrol). ${ }^{1} \mathrm{H}$ NMR $\left(300 \mathrm{MHz}, \mathrm{DMSO}-d_{6}\right) \delta 1.05-1.95(11 \mathrm{H}, \mathrm{m}$, cyclohexyl), $4.30(2 \mathrm{H}$, d, $\left.J=6.1 \mathrm{~Hz}, \mathrm{OCH}_{2}\right), 6.63(1 \mathrm{H}, \mathrm{s}, \mathrm{H}-6), 7.63(2 \mathrm{H}, \mathrm{d}, J=1.2 \mathrm{~Hz}, \mathrm{H}-3)$, $7.81(2 \mathrm{H}, \mathrm{d}, J=1.3 \mathrm{~Hz}, \mathrm{H}-2) .{ }^{13} \mathrm{C}$ NMR $\left(75 \mathrm{MHz}, \mathrm{DMSO}-d_{6}\right) \delta 25.4$, 26.2, 29.0, 36.9, 76.3, 88.8, 107.6, 134.2, 148.1, 152.1, 155.6. LRMS $\left(\mathrm{ES}^{+}\right) \mathrm{m} / z 266.0[\mathrm{M}+\mathrm{H}]^{+}$.

5-Cyclohexylmethoxy-7-anilinoimidazo[1,2-a]pyrimidine (101). The title compound was synthesized according to general procedure A from 100 ( $0.075 \mathrm{mg}, 0.28 \mathrm{mmol})$. Purification by chromatography (silica; EtOAc), followed by crystallization from $\mathrm{MeOH}$, gave 101 (31 $\mathrm{mg}, 34 \%) ; R_{\mathrm{f}} 0.11$ (100\% EtOAc); mp 252-253 ${ }^{\circ} \mathrm{C}$; UV $\lambda_{\max }(\mathrm{EtOH})$ 305, 257, $212 \mathrm{~nm}$. IR $\left(\mathrm{cm}^{-1}\right)$ 3153, 2926, 2847, 2161, 1627, 1595, $1568,1531,1489,1291,1259,1122 .{ }^{1} \mathrm{H}$ NMR (300 MHz, DMSO- $\left.d_{6}\right)$ $\delta 1.05-1.85(11 \mathrm{H}, \mathrm{m}$, cyclohexyl $), 4.35\left(2 \mathrm{H}, \mathrm{d}, J=6.2 \mathrm{~Hz}, \mathrm{OCH}_{2}\right)$, $5.98(1 \mathrm{H}, \mathrm{s}, \mathrm{H}-6), 6.99(1 \mathrm{H}, \mathrm{t}, J=7.3 \mathrm{~Hz}, \mathrm{PhH}), 7.26(1 \mathrm{H}, \mathrm{d}, J=1.6$ $\mathrm{Hz}, \mathrm{H}-3), 7.33(2 \mathrm{H}, \mathrm{dd}, J=7.6,8.2 \mathrm{~Hz}, 2 \times \mathrm{PhH}), 7.44(1 \mathrm{H}, \mathrm{d}, J=1.6$ $\mathrm{Hz}, \mathrm{H}-2), 7.83(2 \mathrm{H}, \mathrm{d}, J=7.7 \mathrm{~Hz}, 2 \times \mathrm{Ph} H), 9.45(1 \mathrm{H}, \mathrm{s}, \mathrm{NH})$. LRMS $\left(\mathrm{ES}^{+}\right) \mathrm{m} / z$ 323.0 $[\mathrm{M}+\mathrm{H}]^{+}$. Anal. Found: C, 70.41; H, 6.59; N, 17.40 . $\mathrm{C}_{19} \mathrm{H}_{22} \mathrm{~N}_{4} \mathrm{O}$ requires $\mathrm{C}, 70.78 ; \mathrm{H}, 6.88 ; \mathrm{N}, 17.38 \%$.

5-Cyclohexylmethoxy-7-(4'-sulfamoylanilino)imidazo[1,2-a]pyrimidine (102). Prepared in accordance with general procedure A from $100(0.25 \mathrm{~g}, 0.94 \mathrm{mmol})$, and purified by chromatography (silica; $5 \% \mathrm{MeOH}$ :EtOAc) to give 102 as a colorless solid (28 mg, 7\%); $R_{\mathrm{f}}$ 0.47 (10\% MeOH:EtOAc); mp 228-229 ${ }^{\circ} \mathrm{C}$. IR $\left(\mathrm{cm}^{-1}\right) 3348,3292$, 2926, 2847, 1653,1617, 1591, 1536, 1429, 1310, 1155. UV $\lambda_{\max }$ (EtOH) 315, 266, $216 \mathrm{~nm} .{ }^{1} \mathrm{H}$ NMR (300 MHz, DMSO- $\left.d_{6}\right) \delta 1.15-$ $1.90(11 \mathrm{H}, \mathrm{m}$, cyclohexyl $), 4.16\left(2 \mathrm{H}, \mathrm{d}, J=6.1 \mathrm{~Hz}, \mathrm{OCH}_{2}\right), 6.03(1 \mathrm{H}$, s, $\mathrm{H}-6), 7.24\left(2 \mathrm{H}, \mathrm{s}, \mathrm{SO}_{2} \mathrm{NH}_{2}\right), 7.32(1 \mathrm{H}, \mathrm{d}, J=1.6 \mathrm{~Hz}, \mathrm{H}-3), 7.51$ $(1 \mathrm{H}, \mathrm{d}, J=1.6 \mathrm{~Hz}, \mathrm{H}-2), 7.78\left(2 \mathrm{H}, \mathrm{d}, J=8.9 \mathrm{~Hz}, \mathrm{H}-2^{\prime}\right.$ and $\left.\mathrm{H}-6^{\prime}\right), 7.99$ $\left(2 \mathrm{H}, \mathrm{d}, J=8.9 \mathrm{~Hz}, \mathrm{H}-3^{\prime}\right.$ and $\left.\mathrm{H}-5^{\prime}\right), 9.85(1 \mathrm{H}, \mathrm{s}, \mathrm{NH}) .{ }^{13} \mathrm{C}$ NMR $(75$ $\left.\mathrm{MHz}, \mathrm{DMSO}-d_{6}\right) \delta 25.45,26.19,29.16,36.85,75.00,78.91,105.83$, $118.42,127.05,131.95,137.03,144.01,154.73,155.68$. LRMS $\left(\mathrm{ES}^{+}\right)$ $\mathrm{m} / z$ 402.0 $[\mathrm{M}+\mathrm{H}]^{+}$; Anal. Found: C, 57.06; H, 5.53; N, 17.09. $\mathrm{C}_{19} \mathrm{H}_{23} \mathrm{~N}_{5} \mathrm{O}_{3} \mathrm{~S}$ requires: C, 56.84; $\mathrm{H}, 5.77 ; \mathrm{N}, 17.44 \%$.

Protein Kinase Assays. Human $\mathrm{CDK} 1 / \mathrm{B}$ was purchased from New England Biolabs or prepared as described, ${ }^{46}$ CDK2/A2 was prepared as described, ${ }^{46} \mathrm{CDK} 4 / \mathrm{D}$ was provided by AstraZeneca, and $\mathrm{CDK} 7 / \mathrm{H}$ and $\mathrm{CDK} 9 / \mathrm{T}$ were purchased from Upstate. $\mathrm{IC}_{50}$ values for $\mathrm{CDK} 1 / \mathrm{B}, \mathrm{CDK} 2 / \mathrm{A}$, and CDK4/D were determined as described. ${ }^{23}$ $\mathrm{IC}_{50}$ values for $\mathrm{CDK} 7 / \mathrm{H}$ and $\mathrm{CDK} 9 / \mathrm{H}$ were determined according to the supplier's instructions (Upstate). CDK1/B, CDK2/A, and CDK4/ $\mathrm{D}$ were assayed at an ATP concentration of $12.5 \mu \mathrm{M}, \mathrm{CDK} 7 / \mathrm{H}$ and CDK9/T at an ATP concentration of $100 \mu \mathrm{M}$. Published $K_{\mathrm{m}}$ (ATP) values for CDK1-cyclin $\mathrm{B}, \mathrm{CDK} 2$-cyclin $\mathrm{A}$, CDK4-cyclin D2, CDK7cyclin H-MAT, and CDK9-cyclin T are $0.8,0.58,3.8,4.1$, and $0.7 \mu \mathrm{M}$, respectively (www.proqinase.com).

Growth Inhibition Assays. The ability of selected compounds to inhibit cell growth was assessed in a panel of human cancer cell lines using an SRB assay. ${ }^{45}$ Briefly, cells in 96-well plates were exposed to inhibitor $(0.1-30 \mu \mathrm{M}$, three replicates per drug concentration) or $0.5 \%$ (v/v) DMSO (for compound 73 treatments) or $0.1 \%(\mathrm{v} / \mathrm{v}$ ) DMSO (for all other inhibitors) for $120 \mathrm{~h}$, stained with sulforhodamine $\mathrm{B}$ and the absorbance at $570 \mathrm{~nm}$ measured (SpectraMax250; Molecular Devices, Wokingham, UK). At least three independent experiments were performed. Growth inhibitory $\mathrm{GI}_{50}$ values were calculated using GraphPad Prism software (GraphPad Software, Inc., San Diego, CA).

Crystallization and Structure Determination. Crystals of CDK2-cyclin A bound to 73 and CDK1-cyclin B-CKS2 bound to 3 were grown as previously described. ${ }^{46}$ Briefly, the inhibitors were added in 2-fold molar excess (which was also a 10-fold higher concentration than the inhibitor $\mathrm{IC}_{50}$ ) to a low concentration solution of the appropriate protein complex and then the samples were concentrated to $10-12 \mathrm{mg} \mathrm{mL}^{-1}$ for crystallization. CDK2-cyclin A73 crystals were grown from a well solution containing $0.6-0.8 \mathrm{M}$ $\mathrm{KCl}, 0.9-1.2 \mathrm{M}\left(\mathrm{NH}_{4}\right)_{2} \mathrm{SO}_{4}$, and $100 \mathrm{mM}$ HEPES ( $\mathrm{pH}$ 7.0). CDK1cyclin B-CKS2 was cocrystallized with 3 from a screen covering the conditions 0.1 M MES/imidazole buffer ( $\mathrm{pH}$ 6.7), 6.5\% MPD, 5\% PEG4K, 10\% PEG1K. Before data collection, crystals were cryoprotected by brief immersion in either $8 \mathrm{M}$ sodium formate (CDK2-cyclin A) or $25 \%$ ethylene glycol (CDK1-cyclin B-CKS2).

Data processing was carried out using XDS, MOSFLM, POINTLESS/AIMLESS, ${ }^{52}$ and other programs of the CCP4 suite, ${ }^{53}$ run through the CCP4i2 GUI. ${ }^{54}$ The structures of the different complexes were solved by molecular replacement using Phaser, ${ }^{55}$ and search models drawn from PDB entries 1QMZ (cyclin A-bound CDK2) and $4 \mathrm{Y} 72$ (CDK1-cyclin B-CKS2). Models of 73 and 3 and ligand restraints were generated using ACEDRG within the CCP4i2 suite, ${ }^{54}$ and built into the electron density using Coot. ${ }^{56}$ Structures were refined using REFMAC, ${ }^{57}$ interspersed with manual rebuilding in Coot, including TLS refinement.

\section{ASSOCIATED CONTENT}

\section{Supporting Information}

The Supporting Information is available free of charge on the ACS Publications website at DOI: 10.1021/acs.jmedchem.6b01254.

Structure of compound $\mathbf{1 0 2}$ and associated crystal data and refinement statistics; ${ }^{1} \mathrm{H}$ NMR spectra for compounds 70, 73, 83, 90, 91, and 98 (PDF)

Molecular formula strings (CSV)

\section{Accession Codes}

CDK2-cyclin A in complex with compound 73 (5LQE), and CDK1-cyclin B-CKS2 in complex with compound 3 (5LQF) have been deposited with the PDB and the atomic coordinates and experimental data will be released upon article publication.

\section{AUTHOR INFORMATION}

\section{Corresponding Authors}

*For chemistry, C.C.: phone, (44) 191208 7060; E-mail, celine.cano@ncl.ac.uk.

*For structural biology, J.A.E.: phone, (44) 191208 4422; fax, (44) 191208 4301; E-mail, jane.endicott@ncl.ac.uk. ORCID

Suzannah J. Harnor: 0000-0003-1646-593X

Céline Cano: 0000-0002-2032-2272 


\section{Present Addresses}

"For M.E.M.N. and J.A.E.: Newcastle Cancer Centre, Northern Institute for Cancer Research, Newcastle University Medical School, Paul O'Gorman Building, Framlington Place, Newcastle upon Tyne NE2 4HH, U.K.

${ }^{\perp}$ For C.R.C.: School of Pharmacy and Biomolecular Sciences, Liverpool John Moores University, Liverpool L3 5UA, U.K..

\section{Author Contributions}

C.R.C. and E.A. contributed equally to the work described in this paper. C.R.C., synthesis and characterization of compounds; E.A., protein expression, crytallization, and structure determination; S.J.H., synthesis and characterization of compounds and manuscript preparation; M.P.M., protein structure determination and interpretation; Benoit Carbain, synthesis and characterization of compounds; I.R.H., experimental design and interpretation; L.K.H., synthesis and characterization of compounds; S.K., protein expression and crystallization; C.J.M., synthesis and characterization of compounds; M.E.M.N., protein structure determination, data analysis and interpretation, and manuscript preparation; D.R.N., project conception, experimental design and interpretation; D.M.T., synthesis and characterization of compounds; M.S., synthesis and characterization of compounds; S.J.T., design, conduct, and interpretation of the cellular studies; L.Z.W., design, conduct, and interpretation of CDK2 inhibition experiments; C.W., synthesis and characterization of compounds; S.R.W., experimental design and interpretation and manuscript preparation; B.T.G., project conception, experimental design and interpretation, data analysis, and manuscript preparation; R.J.G., project conception, experimental design and interpretation, data analysis, and manuscript preparation; J.A.E., experimental design and interpretation and manuscript preparation; C.C., project conception, experimental design and interpretation, data analysis, and manuscript preparation.

\section{Notes}

The authors declare no competing financial interest.

${ }^{\#}$ R.J.G.: Deceased 24 September 2014.

\section{ACKNOWLEDGMENTS}

We thank Cancer Research UK (grant no. C2115/A21421) and the Medical Research Council (grant no. G0901526) for financial support. The use of the EPSRC Mass Spectrometry Service at the University of Wales (Swansea) is also gratefully acknowledged. We also thank beamline staff at The Diamond Light Source who provided excellent facilities for data collection, E. Lowe and A. Basle for assistance with data collection and management, N. Brown for advice, and I. Taylor for technical support.

\section{ABBREVIATIONS USED}

$\mathrm{CDK}$, cyclin-dependent kinase; CKS, cyclin-dependent kinase subunit; GI50, half-maximum growth inhibition; HEPES, 2-[4(2-hydroxyethyl)piperazin-1-yl] ethanesulfonic acid; MES, 2morpholin-4-ylethanesulfonic acid; MPD, 2-methyl-2,4-pentanediol; SRB, sulforhodamine B; TFE, 2,2,2-trifluoroethanol; TLS, translation, libration, and screw-rotation

\section{REFERENCES}

(1) Lim, S.; Kaldis, P. Cdks, Cyclins and CKIs: Roles Beyond Cell Cycle Regulation. Development 2013, 140, 3079-3093.
(2) Malumbres, M. Cyclin-Dependent Kinases. Genome Biol. 2014 $15,122-132$.

(3) Malumbres, M.; Barbacid, M. Cell Cycle, CDKs and Cancer: A Changing Paradigm. Nat. Rev. Cancer 2009, 9, 153-166.

(4) Casimiro, M. C.; Velasco-Velazquez, M.; Aguirre-Alvarado, C.; Pestell, R. G. Overview of Cyclins D1 Function in Cancer and the CDK Inhibitor Landscape: Past and Present. Expert Opin. Invest. Drugs 2014, 23, 295-304.

(5) Sherr, C. J.; Beach, D.; Shapiro, G. I. Targeting CDK4 and CDK6: From Discovery to Therapy. Cancer Discovery 2016, 6, 353367.

(6) Bruyere, C.; Meijer, L. Targeting Cyclin-Dependent Kinases in Anti-Neoplastic Therapy. Curr. Opin. Cell Biol. 2013, 25, 772-779.

(7) Asghar, U.; Witkiewicz, A. K.; Turner, N. C.; Knudsen, E. S. The History and Future of Targeting Cyclin-Dependent Kinases in Cancer Therapy. Nat. Rev. Drug Discovery 2015, 14, 130-146.

(8) Bose, P.; Simmons, G. L.; Grant, S. Cyclin-Dependent Kinase Inhibitor Therapy for Hematologic Malignancies. Expert Opin. Invest. Drugs 2013, 22, 723-738.

(9) Aleem, E.; Arceci, R. J. Targeting Cell Cycle Regulators in Hematologic Malignancies. Front. Cell Dev. Biol. 2015, 3, 16-37.

(10) O’Leary, B.; Finn, R. S.; Turner, N. C. Treating Cancer with Selective CDK4/6 Inhibitors. Nat. Rev. Clin. Oncol. 2016, 13, 417430.

(11) Sánchez-Martínez, C.; Gelbert, L. M.; Lallena, M. J.; de Dios, A. Cyclin Dependent Kinase (CDK) Inhibitors as Anticancer Drugs. Bioorg. Med. Chem. Lett. 2015, 25, 3420-3435.

(12) Tetsu, O.; McCormick, F. Proliferation of Cancer Cells Despite CDK2 Inhibition. Cancer Cell 2003, 3, 233-245.

(13) Ortega, S.; Prieto, I.; Odajima, J.; Martin, A.; Dubus, P.; Sotillo, R.; Barbero, J. L.; Malumbres, M.; Barbacid, M. Cyclin-Dependent Kinase 2 is Essential for Meiosis but not for Mitotic Cell Division in Mice. Nat. Genet. 2003, 35, 25-31.

(14) Berthet, C.; Aleem, E.; Coppola, V.; Tessarollo, L.; Kaldis, P. Cdk2 Knockout Mice are Viable. Curr. Biol. 2003, 13, 1775-1785.

(15) Horiuchi, D.; Huskey, N. E.; Kusdra, L.; Wohlbold, L.; Merrick, K. A.; Zhang, C.; Creasman, K. J.; Shokat, K. M.; Fisher, R. P.; Goga, A. Chemical-Genetic Analysis of Cyclin Dependent Kinase 2 Function Reveals an Important Role in Cellular Transformation by Multiple Oncogenic Pathways. Proc. Natl. Acad. Sci. U. S. A. 2012, 109, E1019E1027.

(16) Cheng, C. K.; Gustafson, W. C.; Charron, E.; Houseman, B. T.; Zunder, E.; Goga, A.; Gray, N. S.; Pollok, B.; Oakes, S. A.; James, C. D.; Shokat, K. M.; Weiss, W. A.; Fan, Q. W. Dual Blockade of Lipid and Cyclin-Dependent Kinases Induces Synthetic Lethality in Malignant Glioma. Proc. Natl. Acad. Sci. U. S. A. 2012, 109, 1272212727.

(17) Deans, A. J.; Khanna, K. K.; McNees, C. J.; Mercurio, C.; Heierhorst, J.; McArthur, G. A. Cyclin-Dependent Kinase 2 Functions in Normal DNA Repair and is a Therapeutic Target in BRCA1Deficient Cancers. Cancer Res. 2006, 66, 8219-8226.

(18) Molenaar, J. J.; Ebus, M. E.; Geerts, D.; Koster, J.; Lamers, F.; Valentijn, L. J.; Westerhout, E. M.; Versteeg, R.; Caron, H. N. Inactivation of CDK2 is Synthetically Lethal to MYCN OverExpressing Cancer Cells. Proc. Natl. Acad. Sci. U. S. A. 2009, 106, 12968-12973.

(19) Yang, L.; Fang, D.; Chen, H.; Lu, Y.; Dong, Z.; Ding, H. F.; Jing, Q.; Su, S. B.; Huang, S. Cyclin-Dependent Kinase 2 is an Ideal Target for Ovary Tumors with Elevated Cyclin E1 Expression. Oncotarget 2015, 6, 20801-20812.

(20) Guha, M. Cyclin-Dependent Kinase Inhibitors Move into Phase III. Nat. Rev. Drug Discovery 2012, 11, 892-894.

(21) Galons, H.; Oumata, N.; Gloulou, O.; Meijer, L. CyclinDependent Kinase Inhibitors Closer to Market Launch? Expert Opin. Ther. Pat. 2013, 23, 945-963.

(22) Santo, L.; Siu, K. T.; Raje, N. Targeting Cyclin-Dependent Kinases and Cell Cycle Progression in Human Cancers. Semin. Oncol. 2015, 42, 788-800. 
(23) Arris, C. E.; Boyle, F. T.; Calvert, A. H.; Curtin, N. J.; Endicott, J. A.; Garman, E. F.; Gibson, A. E.; Golding, B. T.; Grant, S.; Griffin, R. J.; Jewsbury, P.; Johnson, L. N.; Lawrie, A. M.; Newell, D. R.; Noble, M. E.; Sausville, E. A.; Schultz, R.; Yu, W. Identification of Novel Purine and Pyrimidine Cyclin-Dependent Kinase Inhibitors with Distinct Molecular Interactions and Tumor Cell Growth Inhibition Profiles. J. Med. Chem. 2000, 43, 2797-2804.

(24) Davies, T.-G.; Bentley, J.; Arris, C.-E.; Boyle, F. T.; Curtin, N.-J.; Endicott, J.-A.; Gibson, A.-E.; Golding, B.-T.; Griffin, R.-J.; Hardcastle, I.-R.; Jewsbury, P.; Johnson, L.-N.; Mesguiche, V.; Newell, D.-R.; Noble, M.-E. M.; Tucker, J.-A.; Wang, L.; Whitfield, H.-J. StructureBased Design of a Potent Purine-Based Cyclin-Dependent Kinase Inhibitor. Nat. Struct. Biol. 2002, 9, 745-749.

(25) Hardcastle, I. R.; Arris, C. E.; Bentley, J.; Boyle, F. T.; Chen, Y.; Curtin, N. J.; Endicott, J. A.; Gibson, A. E.; Golding, B. T.; Griffin, R. J.; Jewsbury, P.; Menyerol, J.; Mesguiche, V.; Newell, D. R.; Noble, M. E.; Pratt, D. J.; Wang, L. Z.; Whitfield, H. J. N2-Substituted O6Cyclohexylmethylguanine Derivatives: Potent Inhibitors of CyclinDependent Kinases 1 and 2. J. Med. Chem. 2004, 47, 3710-3722.

(26) Marchetti, F.; Cano, C.; Curtin, N. J.; Golding, B. T.; Griffin, R. J.; Haggerty, K.; Newell, D. R.; Parsons, R. J.; Payne, S. L.; Wang, L. Z.; Hardcastle, I. R. Synthesis and Biological Evaluation of 5Substituted O4-Alkylpyrimidines as CDK2 Inhibitors. Org. Biomol. Chem. 2010, 8, 2397-2407.

(27) Jonkers, I.; Lis, J. T. Getting up to Speed with Transcription Elongation by RNA Polymerase II. Nat. Rev. Mol. Cell Biol. 2015, 16, 167-177.

(28) Fischer, P. M.; Gianella-Borradori, A. Recent Progress in the Discovery and Development of Cyclin-Dependent Kinase Inhibitors. Expert Opin. Invest. Drugs 2005, 14, 457-477.

(29) Parry, D.; Guzi, T.; Shanahan, F.; Davis, N.; Prabhavalkar, D.; Wiswell, D.; Seghezzi, W.; Paruch, K.; Dwyer, M. P.; Doll, R.; Nomeir, A.; Windsor, W.; Fischmann, T.; Wang, Y.; Oft, M.; Chen, T.; Kirschmeier, P.; Lees, E. M. Dinaciclib (SCH 727965), A Novel and Potent Cyclin-Dependent Kinase Inhibitor. Mol. Cancer Ther. 2010, 9, 2344-2353.

(30) Pratt, D. J.; Bentley, J.; Jewsbury, P.; Boyle, F. T.; Endicott, J. A.; Noble, M. E. Dissecting the Determinants of Cyclin-Dependent Kinase 2 and Cyclin-Dependent Kinase 4 Inhibitor Selectivity. J. Med. Chem. 2006, 49, 5470-5477.

(31) Gibson, A. E.; Arris, C. E.; Bentley, J.; Boyle, F. T.; Curtin, N. J.; Davies, T. G.; Endicott, J. A.; Golding, B. T.; Grant, S.; Griffin, R. J.; Jewsbury, P.; Johnson, L. N.; Mesguiche, V.; Newell, D. R.; Noble, M. E.; Tucker, J. A.; Whitfield, H. J. Probing the ATP Ribose-Binding Domain of Cyclin-Dependent Kinases 1 and 2 with $\mathrm{O}(6)$-Substituted Guanine Derivatives. J. Med. Chem. 2002, 45, 3381-3393.

(32) Montgomery, J. A.; Hewson, K. Synthesis of Potential Anticancer Agents XX. 2-Fluoropurines. J. Am. Chem. Soc. 1960, 82, 463-468.

(33) Carbain, B.; Coxon, C. R.; Lebraud, H.; Elliott, K. J.; Matheson, C. J.; Meschini, E.; Roberts, A. R.; Turner, D. M.; Wong, C.; Cano, C.; Griffin, R. J.; Hardcastle, I. R.; Golding, B. T. Trifluoroacetic Acid in 2,2,2-Trifluoroethanol Facilitates $S_{N} A r$ Reactions of Heterocycles with Arylamines. Chem. - Eur. J. 2014, 20, 2311-2317.

(34) Lembicz, N. K.; Grant, S.; Clegg, W.; Griffin, R. J.; Heath, S. L.; Golding, B. T. Facilitation of Displacements at the 6-Position of Purines by the Use of 1,4-Diazabicyclo[2.2.2.] octane as Leaving Group. J. Chem. Soc., Perkin Trans. 1 1997, 185-186.

(35) Kato, N.; Sakata, T.; Breton, G.; Le Roch, K. G.; Nagle, A.; Andersen, C.; Bursulaya, B.; Henson, K.; Johnson, J.; Kumar, K. A.; Marr, F.; Mason, D.; McNamara, C.; Plouffe, D.; Ramachandran, V.; Spooner, M.; Tuntland, T.; Zhou, Y.; Peters, E. C.; Chatterjee, A.; Schultz, P. G.; Ward, G. E.; Gray, N.; Harper, J.; Winzeler, E. A. Gene Expression Signatures and Small-Molecule Compounds Link a Protein Kinase to Plasmodium Falciparum Motility. Nat. Chem. Biol. 2008, 4, 347-356.

(36) Molander, G. A.; Gormisky, P. E. Cross-Coupling of Cyclopropyl- and Cyclobutyltrifluoroborates with Aryl and Heteroaryl Chlorides. J. Org. Chem. 2008, 73, 7481-7485.
(37) Temple, C.; Smith, B. H.; Montgomery, J. A. Preparation of 2,5Diamino-4,6-dichloropyrimidine. J. Org. Chem. 1975, 40, 3141-3142.

(38) Shealy, Y. F.; Clayton, J. D.; O’Dell, C. A.; Montgomery, J. A. vTriazolo[4,5-d]pyramidines. II. O-Substituted Derivatives of 8Azaguanine and 8-Azahypoxanthine. J. Org. Chem. 1962, 27, 45184523.

(39) Seela, F.; Steker, H. Synthesis of 2'-Deoxyribofuranosides of 8Aza-7-deazaguanine and Related Pyrazolo[3,4-d]pyrimidines. Helv. Chim. Acta 1986, 69, 1602-1613.

(40) Brückl, T.; Thoma, I.; Wagner, A. J.; Knochel, P.; Carell, T. Efficient Synthesis of Deazaguanosine-Derived tRNA Nucleosides PreQ0, PreQ1, and Archaeosine Using the Turbo-Grignard Method. Eur. J. Org. Chem. 2010, 2010, 6517-6519.

(41) Revankar, G. R.; Matthews, T. R.; Robins, R. K. Synthesis and Antimicrobial Activity of Certain Imidazo[1,2-a]pyrimidines. J. Med. Chem. 1975, 18, 1253-1255.

(42) Harada, H.; Asano, O.; Hoshino, Y.; Yoshikawa, S.; Matsukura, M.; Kabasawa, Y.; Niijima, J.; Kotake, Y.; Watanabe, N.; Kawata, T.; Inoue, T.; Horizoe, T.; Yasuda, N.; Minami, H.; Nagata, K.; Murakami, M.; Nagaoka, J.; Kobayashi, S.; Tanaka, I.; Abe, S. 2-Alkynyl-8-aryl-9methyladenines as Novel Adenosine Receptor Antagonists: Their Synthesis and Structure-Activity Relationships Toward Hepatic Glucose Production Induced Via Agonism of the A(2B) Receptor. J. Med. Chem. 2001, 44, 170-179.

(43) Rao, R. P.; Robins, R. K.; O’Brien, D. E. The Synthesis of Certain 5,7-Dihydroxyimidazo[1,2- $\alpha]$ pyrimidines. J. Heterocycl. Chem. 1973, 10, 1021-1023.

(44) Revankar, R. R.; Robins, R. K. Synthesis and Biological Activity of some Nucleosides Resembling Guanosine: Imidazo[1,2-a]pyrimidine Nucleosides. Ann. N. Y. Acad. Sci. 1975, 255, 166-172.

(45) Thomas, H. D.; Wang, L.-Z.; Roche, C.; Bentley, J.; Cheng, Y.; Hardcastle, I. R.; Golding, B. T.; Griffin, R. J.; Curtin, N. J.; Newell, D. R. Preclinical In Vitro and In Vivo Evaluation of the Potent and Specific Cyclin-Dependent Kinase 2 Inhibitor NU6102 and a Water Soluble Prodrug NU6301. Eur. J. Cancer 2011, 47, 2052-2059.

(46) Brown, N. R.; Korolchuk, S.; Martin, M. P.; Stanley, W. A.; Moukhametzianov, R.; Noble, M. E. M.; Endicott, J. A. CDK1 Structures Reveal Conserved and Unique Features of the Essential Cell Cycle CDK. Nat. Commun. 2015, 6, 6769-6780.

(47) Beach, J. W.; Kim, H. O.; Jeong, L. S.; Nampalli, S.; Islam, Q.; Ahn, S. K.; Babu, J. R.; Chu, C. K. A Highly Stereoselective Synthesis of Anti-HIV 2',3'-Dideoxy- and 2',3'-Didehydro-2' $3^{\prime}$-dideoxynucleosides. J. Org. Chem. 1992, 57, 3887-3894.

(48) Baumgarten, H. E.; Petersen, J. M. Reactions of Amines. V. Synthesis of $\alpha$-Amino Ketones. J. Am. Chem. Soc. 1960, 82, 459-463.

(49) Kos, N. J.; Jongejan, H.; Van Der Plas, H. C. Deamination, Involving Ring Opening, in Reactions of 1-Aminopurinium Mesitylenesulfonates with Methanolic Ammonia. Tetrahedron 1987, 43, 4841-4848.

(50) Balsiger, R. W.; Montgomery, J. A. Synthesis of Potential Anticancer Agents. XXV. Preparation of 6-Alkoxy-2-aminopurines. J. Org. Chem. 1960, 25, 1573-1575.

(51) Griffin, R. J.; Arris, C. E.; Bleasdale, C.; Boyle, F. T.; Calvert, A. H.; Curtin, N. J.; Dalby, C.; Kanugula, S.; Lembicz, N. K.; Newell, D. R; Pegg, A. E.; Golding, B. T. Resistance-Modifying Agents. 8. Inhibition of $\mathrm{O}(6)$-Alkylguanine-DNA Alkyltransferase by $\mathrm{O}(6)$ -

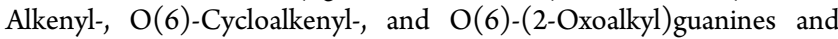
Potentiation of Temozolomide Cytotoxicity In Vitro by $\mathrm{O}(6)-(1-$ Cyclopentenylmethyl)guanine. J. Med. Chem. 2000, 43, 4071-4083.

(52) Evans, P. R. An Introduction to Data Reduction: Space-Group Determination, Scaling and Intensity Statistics. Acta Crystallogr., Sect. D: Biol. Crystallogr. 2011, D67, 282-292.

(53) Winn, M. D.; Ballard, C. C.; Cowtan, K. D.; Dodson, E. J.; Emsley, P.; Evans, P. R.; Keegan, R. M.; Krissinel, E. B.; Leslie, A. G.; McCoy, A. J.; McNicholas, S. J.; Murshudov, G. N.; Pannu, N. S.; Potterton, E. A.; Powell, H. R.; Read, R. J.; Vagin, A. A.; Wilson, K. S. Overview of the CCP4 Suite and Current Developments. Acta Crystallogr., Sect. D: Biol. Crystallogr. 2011, D67, 235-242. 
(54) Potterton, E.; Briggs, P.; Turkenburg, M.; Dodson, E. A Graphical User Interface to the CCP4 Program Suite. Acta Crystallogr., Sect. D: Biol. Crystallogr. 2003, D59, 1131-1137.

(55) McCoy, A. J.; Grosse-Kunstleve, R. W.; Adams, P. D.; Winn, M. D.; Storoni, L. C.; Read, R. J. Phaser Crystallographic Software. J. Appl. Crystallogr. 2007, 40, 658-674.

(56) Emsley, P.; Lohkamp, B.; Scott, W. G.; Cowtan, K. Features and Development of Coot. Acta Crystallogr., Sect. D: Biol. Crystallogr. 2010, D66, 486-501.

(57) Murshudov, G. N.; Vagin, A. A.; Dodson, E. J. Refinement of Macromolecular Structures by the Maximum-Liklihood Method. Acta Crystallogr., Sect. D: Biol. Crystallogr. 1997, D53, 240-255. 